\title{
A Measurement of the Helicity of W Bosons Produced in Top-Quark Decays
}

by

\author{
Nathan J. Goldschmidt \\ A dissertation submitted in partial fulfillment \\ of the requirements for the degree of \\ Doctor of Philosophy \\ (Physics) \\ in The University of Michigan \\ 2005
}

Doctoral Committee:

Associate Professor David W. Gerdes, Chair

Professor Dante E. Amidei

Professor Joel N. Bregman

Professor Gordon L. Kane

Professor Christopher R. Monroe 

(C) Nathan J. Goldschmidt All rights reserved 


\section{DEDICATION}

To my parents, Fred and Barbara Goldschmidt, whom I love. 


\section{ACKNOWLEDGEMENTS}

High-energy physics is a vast and ambitious endeavor. A happy consequence of this is that one has the opportunity to collaborate with many people of the highest quality. Here I mention some of the people who have influenced my work on this dissertation. I am honored to name them all as friends.

I have known Dave Gerdes, my advisor, since I was a student at Johns Hopkins, where he taught my undergraduate E\&M course. He is the best teacher I've known; I thank him for his patience and tolerance, his support and his friendship. I've also known Ken Bloom since my days at Hopkins. Upon our first meeting we understood one another (or at least, he understood me) perfectly. Ken has been an admirable role model and a great friend. The methodology employed in this dissertation was first suggested by Dante Amidei. Dan has consistently offered solid guidance for both research and secular matters, and has been a good friend. This analysis was possible only because of Stephen Miller and Monica Tecchio's work to assay the backgrounds in the data. I thank them

for their friendship. I thank Tom Wright, Alexei Varganov and Claudio Ferretti and for their shrewdness in volleying, sparking, and (sometimes) dismissing ideas.

This analysis was carefully vetted by members of the CDF collaboration. I thank Trevor Vickey, Tony Liss, Jason Nielsen, Robin Erbacher, Jaco Konigsberg and Evelyn Thomson along with members of the "godparent" committee: Phil Schlabach, Patrizia Azzi and Ben Kilminster for maintaining such high standards. You've all helped to make 
me a better physicist. I also sincerely thank Kevin McFarland and Paul Tipton for their attentive criticisms of my analysis.

I've come to know many people at Fermilab whose friendships I value highly. These include Larry Nodulman, Dee and Steve Hahn, Greg Field, Farrukh Azfar, Peter Wittich, Chris Neu, Beate Heinemann, Corrine Mills, Dean Hidas, Ivan Furic, Andreas Korn, Cigdem Issever, Steve Worm, Mark Kruse, Muge Karagoz Unel, Jen Raaf, Michel Sorel, Olga Mena Requejo and Susana Cabrera; Matt Leslie, Nicola Pounder, Martin Griffiths, Sam Harper, Ben Cooper, Gavin Hesketh, Marilyn Audsley Hammond and Sara-madge Wynne; and my friends from the Michigan house Mitch Soderberg, Bo Jayatilaka, Kathy Copic and Heather Ray. I'd also like to acknowledge Fred Niell and Josh Davis from the University of Michigan; they have been great friends to me.

The Dinner Club at Fermilab includes many of the finest people I know. Daniel and Katrine Whiteson I thank for their warmth, compassion and candor. Olga and Andrew Lobban I thank for their exuberance. Mike Kirby I thank for his kindness and his friendship. Eva Halkiadakis I thank for her cheer and laughter. Sondra Rosenberg I thank for her camaraderie. Florencia Canelli I thank for her gravitas and warmth. Ben Kilminster I thank for his humor and sincerity. I thank Brian Connoly for his friendship and altruism. I thank Giulia Manca and Rolf Oldeman for their lightheartedness. I thank Mike Mulhearn and Mila Verdugo, Liz Perez and Chris Hays for their laughter. I thank Robin Erbacher and John Conway for their joviality.

Finally, I thank my family. My parents Barbara and Fred Goldschmidt have always given me support, love and encouragement. I love them very much. To my sister Rebecca I express my affection and admiration. My grandparents Robert and Lilly Funk have always been my greatest fans; they make me proud, and I love them greatly. To my extended family: I am in awe of you all, and am happy to call you family. 


\section{TABLE OF CONTENTS}

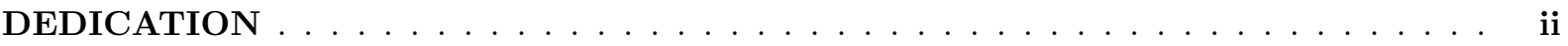

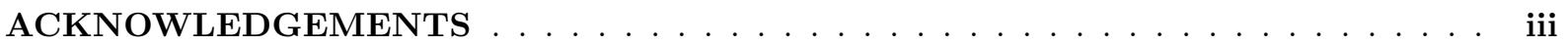

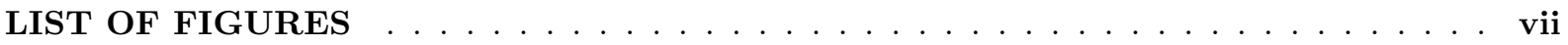

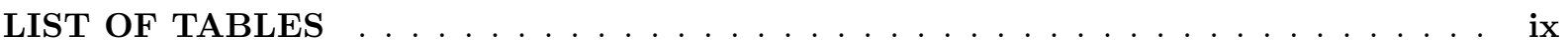

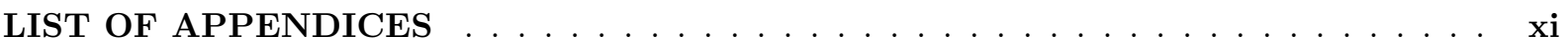

\section{CHAPTER}

1. The Standard Model and phenomenology of the $t W b$ vertex . . . . . . . . . 1

1.1 The Standard Model . . . . . . . . . . . . . . . . . . . . . . . . . 2

1.1.1 Gauge bosons . . . . . . . . . . . . . . . . . . . 2

1.1.2 The Higgs mechanism . . . . . . . . . . . . . . . . . . . 4

1.1.3 Masses of the gauge bosons . . . . . . . . . . . . . . . . . 6

1.1 .4 Fermions . . . . . . . . . . . . . . . . . . 8

1.1.5 Electroweak interactions with fermions . . . . . . . . . . . . . 10

1.2 Top-quark decay . . . . . . . . . . . . . . . . . . . . . . . 13

1.2.1 The fraction of longitudinally-polarized $W$ s from top-quark decays . . . 15

1.2 .2 Non-universal $t W b$ gauge couplings . . . . . . . . . . . . . . . 16

2. Top production and decay $\ldots \ldots \ldots \ldots \ldots \ldots \ldots \ldots$

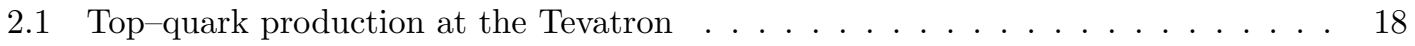

2.2 Experimental signatures of $t \bar{t}$ decay . . . . . . . . . . . . . . . . . . . . 21

2.3 Kinematic variables with helicity information . . . . . . . . . . . . . 25

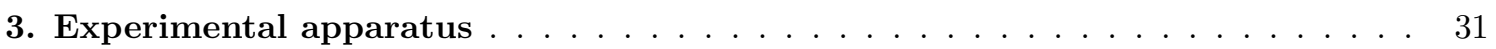

3.1 The Fermilab accelerator complex . . . . . . . . . . . . . . . . . . . . 32

3.1.1 Properties of $p \bar{p}$ collision at the Tevatron . . . . . . . . . . . . . 34

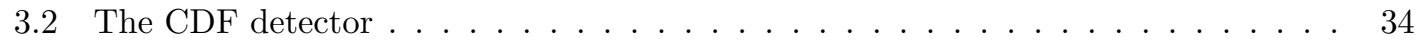

3.2 .1 Charged-particle tracking . . . . . . . . . . . . . 36

3.2 .2 The calorimeter systems . . . . . . . . . . . . . . . . . . . 41

3.2 .3 The muon detectors $\ldots \ldots \ldots \ldots \ldots$

3.2.4 Trigger and data acquisition . . . . . . . . . . . . . . . . . 47

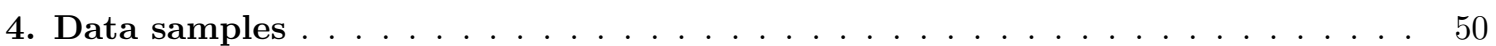

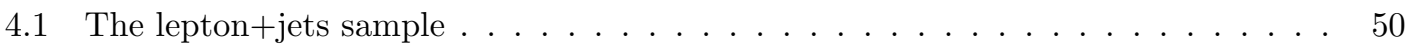

4.1 .1 Event selection . . . . . . . . . . . . . . . . 50

4.1.2 Estimates of background content . . . . . . . . . . . . 55 
4.1.3 Lepton+jets background models . . . . . . . . . . . . . . . . 56

4.1.4 Lepton+jets acceptance ratios . . . . . . . . . . . . . . . . . . . 60

4.1.5 Lepton+jets signal models . . . . . . . . . . . . . . . . . . 61

4.2 The dilepton sample . . . . . . . . . . . . . . . . . . . . . . . . 62

4.2.1 Dilepton background models . . . . . . . . . . . . . . . . 62

4.2 .2 Dilepton acceptance . . . . . . . . . . . . . . . . . 66

5. Method ................................. 67

5.1 The likelihood function . . . . . . . . . . . . . . . . . . . . 67

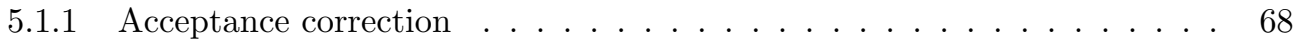

5.1.2 Trigger correction ..................... . . 70

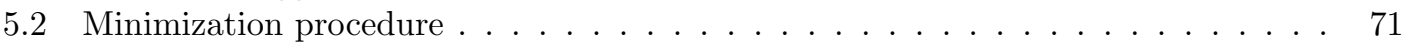

6. Estimates of statistical and systematic uncertainty . . . . . . . . . . . . 73

6.1 The Feldman-Cousins method . . . . . . . . . . . . . . . . . . . . 73

6.1.1 Calculating Feldman-Cousins confidence intervals . . . . . . . . . . . 76

6.1.2 Feldman Cousins resolution functions . . . . . . . . . . . . . . 76

6.2 Resolution function for the lepton+jets analysis . . . . . . . . . . . . . . . . 77

6.3 Resolution function for the dilepton analysis . . . . . . . . . . . . . . . . . . . 77

6.4 Resolution function for the combined analysis . . . . . . . . . . . . . . . . . 80

6.5 Estimates of systematic uncertainty . . . . . . . . . . . . . . . . . 80

6.5.1 Systematics from the signal models . . . . . . . . . . . . . . . 83

6.5.2 Systematics from the background models . . . . . . . . . . . . 84

7. Results . . . . . . . . . . . . . . . . . . . . . . . . . 89

7.1 Result from the lepton+jets analysis . . . . . . . . . . . . . . . . . . . . 89

7.2 Result from the dilepton analysis . . . . . . . . . . . . . . . . . . . 90

7.3 Result from the combined analysis . . . . . . . . . . . . . . . . . . . 91

8. Conclusions . . . . . . . . . . . . . . . . . . . . . . . . . . . . 98

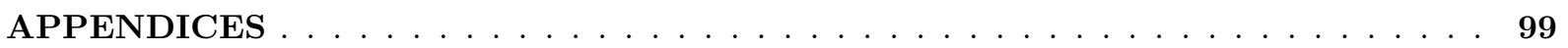

BIBLIOGRAPHY . . . . . . . . . . . . . . . . . . . . . . . . 107 


\section{LIST OF FIGURES}

\section{Figure}

$1.1 \quad$ Feynman diagram for the decay $t \rightarrow W^{+} b \ldots \ldots \ldots \ldots \ldots$

$1.2 \quad$ Feynman diagram for the decay $t \rightarrow \phi^{+} b \ldots \ldots \ldots \ldots \ldots$

$2.1 \quad$ Feynman diagrams of the leading order $t \bar{t}$ pair production processes . . . . . . . . . 19

2.2 Parton distribution functions at (a) $Q^{2}=2$ and (b) $Q^{2}=100 \mathrm{GeV} \ldots \ldots \ldots$

2.3 A summary of the top-quark pair production cross section measurements performed by the $\mathrm{CDF}$ collaboration in run II . . . . . . . . . . . . . . . . . . . . . . 22

2.4 A summary of the top-quark pair production cross section measurements performed by the $\mathrm{D} \emptyset$ collaboration in run II . . . . . . . . . . . . . . . . . . . . . . 23

2.5 The angle between the charged-lepton three-momentum and the $W$ boost vector in the $W$ rest frame . . . . . . . . . . . . . . . . . . . . . . . 25

$2.6 \quad$ Differential decay rates for the process $W_{\lambda} \rightarrow \ell \nu_{\ell} \ldots \ldots \ldots \ldots \ldots$

2.7 Distributions of the transverse momentum of charged leptons from leptonically decaying $W$ bosons in $t \bar{t}$ events . . . . . . . . . . . . . . . . . . . . . . . 29

$3.1 \quad$ A schematic representation of the Fermilab accelerator complex . . . . . . . . . . 32

$3.2 \quad$ A cross-section view of the $\mathrm{CDF}$ detector $\ldots \ldots \ldots \ldots \ldots$

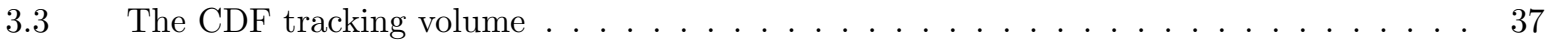

$3.4 \quad$ A transverse view of the $\mathrm{CDF}$ silicon detectors $\ldots \ldots \ldots \ldots$

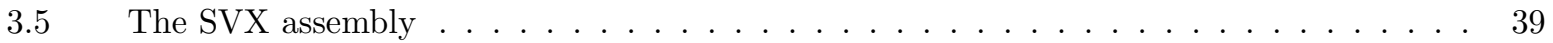

3.6 Transverse views of typical COT cells and the COT endplate . . . . . . . . . . 40

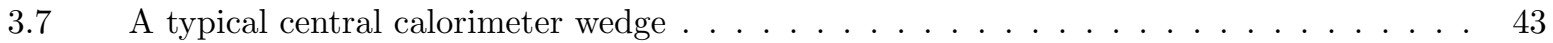

$3.8 \quad$ A map of the coverage of the CDF muon systems $\ldots \ldots \ldots \ldots$

3.9 Transverse views of the central muon detector elements . . . . . . . . . . . . . 46

4.1 Efficiency to tag a $b$-jet as a function of jet $E_{T} \ldots \ldots \ldots \ldots$

4.2 Data/Monte Carlo scale factor for tagging a $b$-jet, as a function of jet $E_{T} \ldots \ldots$ 
4.3 Distribution of charged-lepton $p_{T}$ in the $\geq 3$ jet SECVTX-tagged lepton+jets sample . 57

4.4 Parametrizations of the total expected backgrounds in the double-tag lepton+jet samples 58

4.5 Parametrizations of the total expected backgrounds in the single-tag lepton+jetsamples 59

4.6 Distribution of charged-lepton $p_{T}$ from the inclusive dilepton sample . . . . . . . . . 64

4.7 Parametrizations of the total expected backgrounds in the dilepton samples . . . . . . 65

5.1 Parametrization of the efficiency to trigger on a central electron as a function of the

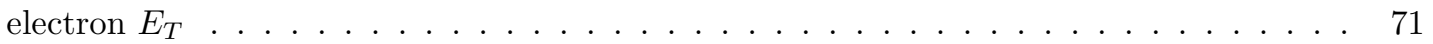

5.2 Parametrization of the efficiency to trigger on a plug electron as a function of the electron

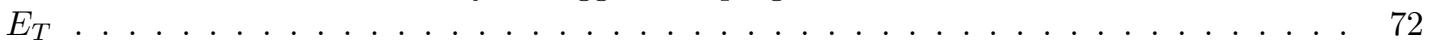

6.1 Toy Feldman-Cousins confidence belts at the $68.3 \%, 95.4 \%$ and $99.7 \%$ confidence levels . 75

$6.2 \quad$ Ensemble tests for the lepton+jets only analysis . . . . . . . . . . . . . . . . 78

6.3 Ensemble tests for the dilepton-only analysis . . . . . . . . . . . . . . . . . 79

6.4 Ensemble tests for the combined analysis . . . . . . . . . . . . . . . . . 81

$7.1 \quad$ Result of the leptontjets analysis . . . . . . . . . . . . . . . . . . . 92

7.2 Feldman-Cousins belts for the lepton+jets analysis . . . . . . . . . . . . . 93

7.3 Result of the dilepton analysis . . . . . . . . . . . . . . . . . . . . 94

7.4 Feldman-Cousins belts for the dilepton analysis . . . . . . . . . . . . . . 95

$7.5 \quad$ Result of the combined analysis . . . . . . . . . . . . . . . . . . 96

7.6 Feldman-Cousins belts for the combined analysis . . . . . . . . . . . . . . 97 


\section{LIST OF TABLES}

\section{Table}

1.1 The gauge boson mass and their associated interactions . . . . . . . . . . . . 7

1.2 The quarks and leptons of the SM and their masses . . . . . . . . . . . . . 8

1.3 Quantum numbers of the SM quarks and leptons . . . . . . . . . . . . . 10

3.1 Tevatron operational parameters from July 2001 through September 2003 . . . . . . 34

3.2 Properties of the CDF II calorimeter systems . . . . . . . . . . . . . . . . . 42

4.1 Yield of events in the $161 \mathrm{pb}^{-1}$ lepton+jets sample . . . . . . . . . . . . . 56

4.2 Sample composition estimate of the 1 SECVTX-tag CEM+jets sample . . . . . . . 60

4.3 Sample composition estimate of the $\geq 2$ SECVTX-tag CEM+jets sample . . . . . . . 60

4.4 Estimated background content of the SECVTX-tag CMUP/CMX+jets sample . . . . . 61

4.5 Estimated background content of the $\geq 2$ SECVTX-tag CMUP/CMX+jets sample . . . 61

4.6 Parametrizations of the total expected background in the double-tag samples . . . . . 62

4.7 Parametrizations of the total expected background in the single-tag samples . . . . . . 62

4.8 Acceptance ratios for the lepton +3 jet samples . . . . . . . . . . . . . . 63

4.9 Acceptance ratios for the lepton +4 jet samples . . . . . . . . . . . . . 63

$4.10 \quad$ Parametrization of the signal PDFs . . . . . . . . . . . . . . . . . . . 63

4.11 Background composition estimates for the dilepton samples . . . . . . . . . . . 66

4.12 Acceptances for the dilepton samples . . . . . . . . . . . . . . . . . . 66

6.1 Shifts in $\hat{F}_{0}$ due to variations in the normalizations of lepton+jets backgrounds in the lepton+jets measurement ... . . . . . . . . . . . . . . . . . 85

6.2 Shifts in $\hat{F}_{0}$ due to variations in the normalizations of lepton+jets backgrounds in the combined measurement . . . . . . . . . . . . . . . . . . . 86

6.3 Estimates of systematic uncertainty in the lepton+jets analysis . . . . . . . . . . 87

6.4 Estimates of systematic uncertainty in the dilepton analysis . . . . . . . . . . . 87 
$6.5 \quad$ Estimates of systematic uncertainty in the combined analysis $\ldots \ldots \ldots$. . . . 88 


\section{LIST OF APPENDICES}

\section{$\underline{\text { Appendix }}$}

A. Notation . . . . . . . . . . . . . . . . . . . . . . 100

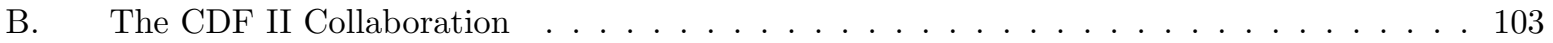




\section{CHAPTER 1}

\section{The Standard Model and phenomenology of the $t W b$ vertex}

Much of our present understanding of nature is articulated in a theory known as the Standard Model of Particle Physics (SM). This remarkably successful theory includes a complete description of nearly all phenomena observed at high-energy physics experiments, including the electromagnetic, weak, and strong nuclear forces. However, there are observed phenomena which are not described by the SM; also, the SM is theoretically inconsistent with general relativity, Einstein's well-tested theory of gravity. Because of these experimental and theoretical puzzles, the SM is not regarded as a fundamental theory; a truly fundamental theory must explain all phenomena observed in nature.

One of the great puzzles of the SM is the origin and nature of electroweak symmetry breaking (EWSB), the mechanism which is thought to imbue the weak bosons and the fermions of the SM with mass. Because of the large masses of the top quark, the $W$ boson and the bottom quark, the $W t b$ interaction vertex is a natural place to explore hypotheses concerning EWSB.

In this chapter we present a brief description of the Standard Model $[1,2,3,4,5]$ and discuss some of the phenomenology of top-quark decays. Appendix A describes the conventions used in this chapter. 


\subsection{The Standard Model}

The Standard Model is a gauge field theory, locally invariant under transformations in the Lie group

$$
G=\mathrm{SU}(3)_{\mathrm{C}} \times \mathrm{SU}(2)_{\mathrm{L}} \times \mathrm{U}(1)_{\mathrm{Y}}
$$

The $\mathrm{SU}(3)_{\mathrm{C}}$ symmetry defines the structure of the strong nuclear interactions, as described by the theory of quantum chromodynamics $(\mathrm{QCD})[6,7]$. The $\mathrm{SU}(2)_{\mathrm{L}} \times \mathrm{U}(1)_{\mathrm{Y}}$ symmetry defines the structure of the unified weak and electromagnetic interactions, as described by the Glashow-Salam-Weinberg (GSW) theory $[8,9,10,11]$.

Masses arise in a gauge-invariant way, due to a process known as the Higgs mechanism $[12,13]$. In this mechanism, the local $\mathrm{SU}(2)_{L} \times \mathrm{U}(1)_{Y}$ symmetry of the electroweak interaction is hidden or "spontaneously broken," and the $\mathrm{U}(1)_{Q}$ symmetry of the electromagnetic interaction emerges. This aspect of the theory correctly predicts the existence of the weak gauge bosons as well as the ratio of their masses [8, 9]. It also predicts the existence of a spin-0 particle: the Higgs boson $(H)$, which has not yet been observed experimentally. We discuss the Higgs mechanism in greater detail in Section 1.1.2.

The interactions of the SM are defined by the Lagrangian density

$$
\mathcal{L}=\mathcal{L}_{g k}+\mathcal{L}_{\phi}+\mathcal{L}_{f k}+\mathcal{L}_{\text {Yukawa }}
$$

We describe Equation 1.2 in terms of the spin-0 scalar fields (the Higgs doublet $\phi$ ), the

spin- $-\frac{1}{2}$ matter fields, (the fermions) and the spin-1 interaction fields (the gauge bosons).

\subsubsection{Gauge bosons}

In the Standard Model, interactions are mediated by the exchange of spin-1 gauge bosons. The group structure of the theory requires that there exist twelve such gauge bosons, one for each generator of the symmetry group $G$. 
Generators of the $\mathrm{U}(1)$ symmetry group are real scalar constants. We identify the generator of the $\mathrm{U}(1)_{Y}$ subgroup of $G$ as the weak hypercharge $(Y)$. The gauge field associated with $\mathrm{U}(1)_{Y}$ is labeled $B_{\mu}$.

The three generators of $\mathrm{SU}(2)$ in the fundamental representation are the $2 \times 2$ Pauli matrices $\vec{\tau}=\left(\tau^{1}, \tau^{2}, \tau^{3}\right)$. The weak isospin operator is

$$
T_{i}=\frac{1}{2} \tau_{i}
$$

The magnitude of the weak isospin $(T)$ and its third component $\left(T_{3}\right)$ are the quantum numbers associated with the $\mathrm{SU}(2)$ symmetry. The gauge boson fields associated with $\mathrm{SU}(2)_{L}$ are labeled $A_{\mu}^{1}, A_{\mu}^{2}$ and $A_{\mu}^{3}$.

The eight generators of $\mathrm{SU}(3)$ in the fundamental representation are the $3 \times 3$ GellMann matrices $\vec{\lambda}=\left(\lambda^{i}, \ldots, \lambda^{8}\right)$. The quantum numbers associated with the $\mathrm{SU}(3)$ subgroup of $G$ are the so-called color charges of QCD (i.e. red, green and blue). The gauge bosons associated with $\mathrm{SU}(3)$ are labeled $G_{\mu}^{1}, \ldots, G_{\mu}^{8}$.

Generators of each of the symmetry subgroups of $G$ commute with the generators of the other subgroups. Therefore, the kinetic energy terms of the SM Lagrangian which describe self-interactions of the gauge fields are

$$
\mathcal{L}_{g k}=-\frac{1}{4} A_{\mu \nu}^{i} A^{i \mu \nu}-\frac{1}{4} B_{\mu \nu} B^{\mu \nu}
$$

where

$$
\begin{gathered}
A_{\mu \nu}^{i}=\partial_{\mu} A_{\nu}^{i}-\partial_{\nu} A_{\mu}^{i}-g \epsilon^{i j k} A_{\mu}^{j} A_{\nu}^{k} \\
B_{\mu \nu}=\partial_{\mu} B_{\nu}-\partial_{\nu} B_{\mu}
\end{gathered}
$$

Here $g$ is the weak coupling constant, and $\epsilon^{i j k}$ is the structure constant for $\mathrm{SU}(2)$. 


\subsubsection{The Higgs mechanism}

If the SM is to be gauge-invariant and renormalizable, its Lagrangian cannot contain explicit mass terms. Instead, masses are introduced via the Higgs mechanism [12, 13], which hides (or spontaneously breaks) the electroweak symmetry and does not spoil the gauge invariance or the renormalizability of the massless theory $[14,15,16]$.

A multiplet of scalar fields (called the Higgs field) is introduced: these fields couple to the massless $\mathrm{SU}(2)_{L} \times \mathrm{U}(1)_{Y}$ gauge bosons and the massless fermions, but leave the Lagrangian invariant under $\mathrm{SU}(2) \times \mathrm{U}(1)$ gauge transformations. The minimum energy states of the Higgs field are degenerate, and one particular state is spontaneously chosen as the vacuum. This choice does not affect the resulting physics, but when the Lagrangian is expressed in terms of this arbitrary vacuum state, the $\mathrm{SU}(2)_{L} \times \mathrm{U}(1)_{Y}$ symmetry is reduced to the familiar $\mathrm{U}(1)_{Q}$ symmetry of the electromagnetic interaction, and the gauge bosons associated with the now-hidden symmetries mix and acquire mass.

The Higgs field is an $\mathrm{SU}(2)$ doublet composed of complex scalar fields

$$
\phi=\left(\begin{array}{c}
\phi^{+} \\
\phi^{0}
\end{array}\right)=\frac{1}{\sqrt{2}}\left(\begin{array}{c}
\phi_{1}+i \phi_{2} \\
\phi_{3}+i \phi_{4}
\end{array}\right),
$$

where $\phi_{1} \ldots \phi_{4}$ are real scalar fields. The fields $\phi^{+}$and $\phi^{0}$ are assigned quantum numbers

$$
\begin{aligned}
\phi^{+}: & T=\frac{1}{2}, & T_{3}=\frac{1}{2}, & Y=1, & Q=+1, \\
\phi^{0}: & T=\frac{1}{2}, & T_{3}=-\frac{1}{2}, & Y=1, & Q=0 .
\end{aligned}
$$

The dynamics of the Higgs field is described by the $\mathcal{L}_{\phi}$ terms of the SM Lagrangian

$$
\mathcal{L}_{\phi}=\left(D_{\mu} \phi\right)^{\dagger}\left(D^{\mu} \phi\right)-V(\phi)
$$

The scalar potential is

$$
V(\phi)=\mu^{2} \phi^{\dagger} \phi+\lambda\left(\phi^{\dagger} \phi\right)^{2}
$$


and the covariant derivative is ${ }^{1}$

$$
D_{\mu}=\partial_{\mu}-i g \frac{\tau^{j}}{2} A_{\mu}^{j}-i g^{\prime} \frac{Y}{2} B_{\mu}
$$

For the case $\mu^{2}<0$ and $\lambda>0$, the potential $V(\phi)$ is minimized if

$$
\phi^{\dagger} \phi=\frac{\phi_{1}^{2}+\phi_{2}^{2}+\phi_{3}^{2}+\phi_{4}^{2}}{2}=\frac{-\mu^{2}}{2 \lambda} \equiv \frac{v^{2}}{2} .
$$

The scalar Lagrangian $\mathcal{L}_{\phi}$ is invariant under $\mathrm{SU}(2) \times \mathrm{U}(1)$ gauge transformations of the Higgs field; we are therefore free to choose a gauge by applying such a transformation. It's convenient to work in the unitary gauge, where three of the four degrees of freedom of the Higgs field vanish (e.g. $\phi_{1}=\phi_{2}=\phi_{4}=0$ ). The vacuum state is then

$$
\phi_{0}=\frac{1}{\sqrt{2}}\left(\begin{array}{l}
0 \\
v
\end{array}\right)
$$

Still working in the unitary gauge, we can express excitations of the Higgs field relative to the vacuum state

$$
\phi(x)=\frac{1}{\sqrt{2}}\left(\begin{array}{c}
0 \\
v+H(x)
\end{array}\right),
$$

where $H(x)$ is the Higgs boson, manifested by excitations of the Higgs field from the vacuum expectation value $v$. Since we are able to "gauge away" all but one of the four scalars of the Higgs doublet, only one can appear as a real particle, i.e. the Higgs boson. The other three fields are the Goldstone bosons, which are not manifest as independent physical particles, but appear as the longitudinal polarization states of the soon-to-be massive gauge bosons. We give a concrete example of this relationship in Section 1.2.

\footnotetext{
${ }^{1}$ The Higgs field couples only to the $\mathrm{SU}(2)_{L} \times \mathrm{U}(1)_{Y}$ gauge bosons; the $\mathrm{SU}(3)_{C}$ symmetry is unaffected by the Higgs mechanism.
} 


\subsubsection{Masses of the gauge bosons}

If we re-write the Higgs sector terms of the SM Lagrangian using Equation (1.14), the scalar potential becomes

$$
V=\frac{1}{2}\left(2 \lambda v^{2}\right) H^{2}(x)+\lambda v H^{3}(x)+\frac{1}{4} \lambda H^{4}(x)
$$

and the Higgs boson has acquired a mass,

$$
m_{H}=v \sqrt{2 \lambda}
$$

The covariant derivative of Equation (1.11) mixes the $\mathrm{SU}(2)_{L}$ and $\mathrm{U}(1)_{Y}$ gauge fields. Evaluating at the vacuum state, we find

$$
\begin{aligned}
D_{\mu} \phi_{0} & =\frac{1}{\sqrt{2}}\left(\partial_{\mu}-i \frac{g}{2} \tau^{i} W_{\mu}^{i}-i \frac{g^{\prime}}{2} B_{\mu}\right)\left(\begin{array}{l}
0 \\
v
\end{array}\right) \\
& =-\frac{i v}{2 \sqrt{2}}\left(\begin{array}{c}
g\left(A_{\mu}^{1}-i A_{\mu}^{2}\right) \\
g^{\prime} B_{\mu}-g A_{\mu}^{3}
\end{array}\right)
\end{aligned}
$$

We can identify the mass eigenstates of the gauge bosons by examining the kinetic terms of the scalar field,

$$
\left(D_{\mu} \phi_{0}\right)^{\dagger}\left(D^{\mu} \phi_{0}\right)=\frac{v^{2}}{8}\left(g^{2} W_{\mu}^{+} W^{-\mu}+\left(g^{2}+g^{\prime 2}\right) Z_{\mu} Z^{\mu}\right)
$$

The massive gauge bosons are the $W^{ \pm}$and the $Z^{0}$

$$
\begin{aligned}
W_{\mu}^{ \pm} & =\frac{1}{\sqrt{2}}\left(A_{\mu}^{1} \mp i A_{\mu}^{2}\right) \\
Z_{\mu}^{0} & =\frac{1}{\sqrt{g^{2}+g^{\prime 2}}}\left(g A_{\mu}^{3}-g^{\prime} B_{\mu}\right)
\end{aligned}
$$

which have masses

$$
\begin{aligned}
m_{W} & =\frac{v}{2} g \\
m_{Z} & =\frac{v}{2} \sqrt{g^{2}+g^{2}}
\end{aligned}
$$




\begin{tabular}{lccr}
\hline Particle & Symbol & Mass $\left(\mathrm{GeV} / c^{2}\right)$ & Interaction \\
\hline Photon & $\gamma$ & 0 & Electromagnetic \\
$W$ boson & $W^{ \pm}$ & 80.4 & Weak charged-current \\
$Z$ boson & $Z^{0}$ & 91.2 & Weak neutral-current \\
gluon & $G^{a}$ & 0 & Strong \\
\hline
\end{tabular}

Table 1.1: The gauge boson mass and their associated interactions.

The fourth mass eigenstate is the photon

$$
A_{\mu}^{0}=\frac{1}{\sqrt{g^{2}+g^{\prime 2}}}\left(g^{\prime} A_{\mu}^{3}+g B_{\mu}\right)
$$

which is associated with the electric charge operator

$$
Q \equiv T_{3}+Y \text {. }
$$

Table 1.1 lists the physical gauge bosons of the Standard Model and their masses.

The vacuum state of the Higgs field is invariant under the gauge transformation

$$
\phi_{0} \rightarrow \phi_{0}{ }^{\prime}=\exp \left[i \alpha(x)\left(T_{3}+Y\right)\right] \phi_{0} .
$$

Therefore the photon acquires no mass, the vacuum carries no electric charge, and the electroweak symmetry has been reduced,

$$
\mathrm{SU}(2)_{L} \times \mathrm{U}(1)_{Y} \rightarrow \mathrm{U}(1)_{Q}
$$

The mixing of the $\mathrm{SU}(2)_{L}$ and $\mathrm{U}(1)_{Y}$ gauge bosons is parametrized by the weak mixing angle $\theta_{w}$

$$
c_{w} \equiv \cos \theta_{w}=\frac{g}{\sqrt{g^{2}+g^{\prime 2}}}, \quad s_{w} \equiv \sin \theta_{w}=\frac{g^{\prime}}{\sqrt{g^{2}+g^{\prime 2}}}
$$

such that

$$
\left(\begin{array}{c}
Z_{\mu} \\
A_{\mu}
\end{array}\right)=\left(\begin{array}{cc}
\cos \theta_{W} & -\sin \theta_{W} \\
\sin \theta_{W} & \cos \theta_{W}
\end{array}\right)\left(\begin{array}{c}
W_{\mu}^{3} \\
B_{\mu}
\end{array}\right) .
$$




\begin{tabular}{|c|c|c|c|c|c|}
\hline \multicolumn{2}{|c|}{ First Generation } & \multicolumn{2}{|c|}{ Second Generation } & \multicolumn{2}{|c|}{ Third Generation } \\
\hline \multicolumn{6}{|c|}{ Quarks } \\
\hline up $(u)$ & $1.5-4.0 \mathrm{MeV}$ & charm $(c)$ & $1.15-1.35 \mathrm{GeV}$ & top $(t)$ & $178 \pm 4.3 \mathrm{GeV}$ \\
\hline down $(d)$ & $4-8 \mathrm{MeV}$ & strange $(s)$ & $80-130 \mathrm{MeV}$ & bottom $(b)$ & $4.1-4.4 \mathrm{GeV}$ \\
\hline \multicolumn{6}{|c|}{ Leptons } \\
\hline electron $(e)$ & $0.5 \mathrm{MeV}$ & muon $(\mu)$ & $106 \mathrm{MeV}$ & $\operatorname{tau}(\tau)$ & $1.8 \mathrm{GeV}$ \\
\hline electron neutrino $\left(\nu_{e}\right)$ & $<3 \mathrm{eV}$ & muon neutrino $\left(\nu_{\mu}\right)$ & $<0.19 \mathrm{MeV}$ & tau neutrino $\left(\nu_{\tau}\right)$ & $<18.2 \mathrm{MeV}$ \\
\hline
\end{tabular}

Table 1.2: The quarks and leptons of the SM, their symbols (in parentheses) and their observed masses [17, $18]$.

We can write the covariant derivative (1.11) in terms of the physically observable gauge bosons. We identify the isospin raising and lowering operators

$$
T_{ \pm} \equiv\left(T_{1} \pm i T_{2}\right)
$$

and the electron charge

$$
e=\frac{g g^{\prime}}{\sqrt{g^{2}+g^{\prime 2}}}
$$

The covariant derivative is then

$$
D_{\mu}=\partial_{\mu}-i \frac{e}{\sqrt{2} s_{w}}\left(W_{\mu}^{+} T_{+}+W_{\mu}^{-} T_{-}\right)-i \frac{e}{s_{w} c_{w}} Z_{\mu}\left(T_{3}-s_{w}^{2} Q\right)-i e A_{\mu} Q
$$

\subsubsection{Fermions}

The matter spectrum of the SM of consists of three generations of spin- $\frac{1}{2}$ fields. Each generation is a "copy" of the others with respect to their gauge quantum numbers, but all differ with respect to their masses. A generation consists of one charged and one neutral lepton, which are subject to the electroweak interaction, and one up-type and one downtype quark, which are subject to electroweak and strong interactions. Table 1.2 lists the fermions of the SM and their observed masses.

To be consistent with experimental results, the fermion matter fields of the SM are constructed to have a unique chiral structure. The charged-current weak interaction appears to couple only to left-handed (i.e. negative helicity) fermions and right-handed 
(i.e. positive helicity) antifermions. This is accommodated in the theory by choosing two different representations of $\mathrm{SU}(2)$ for left- and right-handed fermions. Left-handed fermions appear as $\mathrm{SU}(2)$ doublets. The three generations of left-handed leptons are

$$
E_{L}^{i}=\left(\begin{array}{c}
\nu^{i} \\
\ell^{i}
\end{array}\right)_{L}=\left(\left(\begin{array}{l}
\nu_{e} \\
e
\end{array}\right)_{L},\left(\begin{array}{c}
\nu_{\mu} \\
\mu
\end{array}\right)_{L},\left(\begin{array}{c}
\nu_{\tau} \\
\tau
\end{array}\right)_{L}\right) .
$$

The three generations of left-handed quarks are

$$
Q_{L}^{i}=\left(\begin{array}{l}
u^{i} \\
d^{i}
\end{array}\right)_{L}=\left(\left(\begin{array}{l}
u \\
d
\end{array}\right)_{L},\left(\begin{array}{l}
c \\
s
\end{array}\right)_{L},\left(\begin{array}{l}
t \\
b
\end{array}\right)_{L}\right) .
$$

Right-handed fermions appear as SU(2) singlets. The right-handed charged leptons are

$$
\ell_{R}^{i}=\left(e_{R}, \mu_{R}, \tau_{R}\right)
$$

The right-handed up- and down-type quarks are

$$
u_{R}^{i}=\left(u_{R}, c_{R}, t_{R}\right)
$$

and

$$
d_{R}^{i}=\left(d_{R}, s_{R}, b_{R}\right)
$$

By construction, there are no right-handed neutrino states in the SM. However, recent experimental results indicate neutrinos are not massless [19, 20]; therefore right-handed neutrino states should be included in the theory. The experimental picture of the neutrino spectrum is far from clear, though. While we know that the number of light neutrino flavors is three [17], we do not know if neutrinos are Majorana or Dirac particles, or if there exist additional massive sterile neutrinos, or if other "exotic" scenarios describe the neutrino sector. 


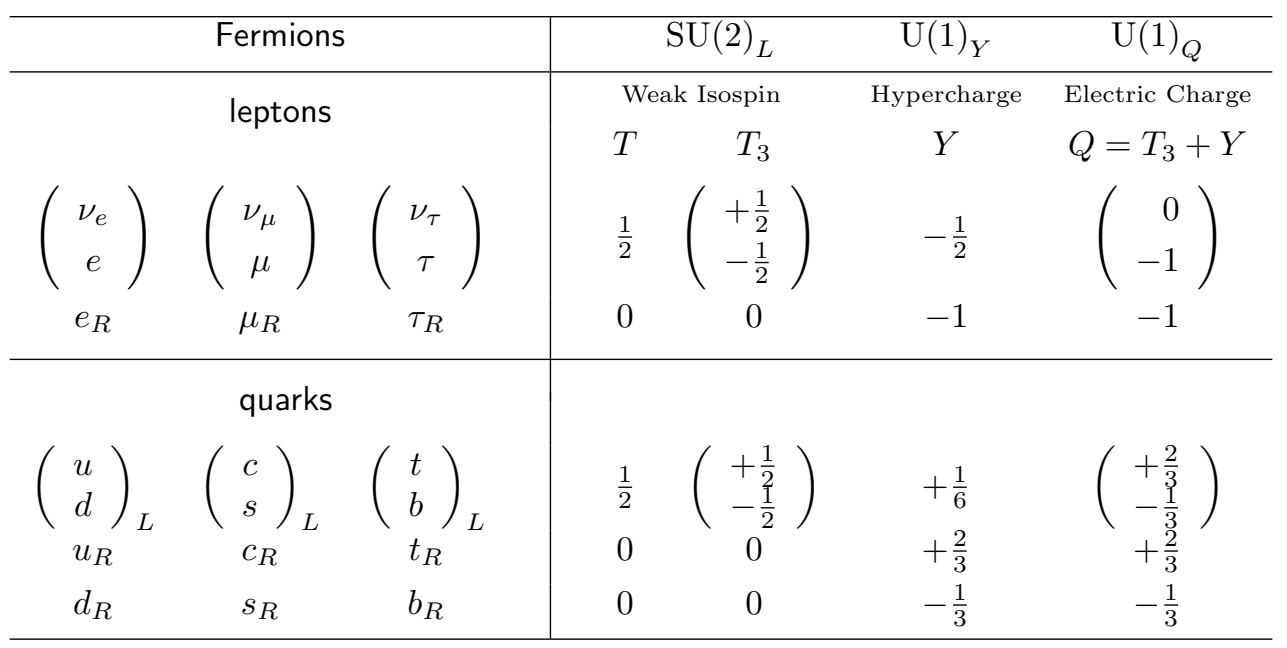

Table 1.3: Quantum numbers of the SM quarks and leptons.

Only the quarks participate in the strong nuclear interaction; each quark flavor carries a color charge (red $(r)$, green $(g)$, or blue $(b))$ and transforms as a triplet under $\mathrm{SU}(3)$ :

$$
q=\left(\begin{array}{c}
q_{r} \\
q_{g} \\
q_{b}
\end{array}\right) .
$$

Table 1.3 lists the gauge quantum numbers of the SM fermions.

\subsubsection{Electroweak interactions with fermions}

The Lagrangian of electroweak interactions, ignoring fermion masses, follows directly from the above quantum number assignments. The kinetic terms are

$$
\mathcal{L}=\sum_{i=1}^{i=3} \bar{E}_{L}^{i}(i \not D) E_{L}^{i}+\bar{\ell}_{R}^{i}(i \not D) \ell_{R}^{i}+\bar{Q}_{L}^{i}(i \not D) Q_{L}^{i}+\bar{u}_{R}^{i}(i \not D) u_{R}^{i}+\bar{d}_{R}^{i}(i \not D) d_{R}^{i}
$$

where $D_{\mu}$ is the covariant derivative given in Equation (1.11), with $T_{3}$ and $Y$ evaluated according to the field on which they act. In terms of the mass eigenstates of the gauge bosons, the Lagrangian describing the electroweak interactions of the $i^{\text {th }}$ generation 
fermions is

$$
\begin{aligned}
\mathcal{L}=\sum_{i=1}^{i=3}\left\{\bar{E}_{L}^{i}(i \not \partial) E_{L}^{i}+\bar{\ell}_{R}^{i}(i \not \partial)\right. & \ell_{R}^{i}+\bar{Q}_{L}^{i}(i \not \partial) Q_{L}^{i}+\bar{u}_{R}^{i}(i \not \partial) u_{R}^{i}+\bar{d}_{R}^{i}(i \not \partial) d_{R}^{i} \\
& \left.+g\left(W_{\mu}^{+} J_{W^{+}}^{\mu, i}+W_{\mu}^{-} J_{W^{-}}^{\mu, i}+Z_{\mu}^{0} J_{Z}^{\mu, i}\right)+e A_{\mu} J_{E M}^{\mu, i}\right\} .
\end{aligned}
$$

The weak charged- and neutral currents are respectively

$$
\begin{aligned}
J_{W^{+}}^{\mu, i} & =\frac{1}{\sqrt{2}}\left(\bar{\nu}^{i}{ }_{L} \gamma^{\mu} \ell_{L}^{i}+\bar{u}^{i}{ }_{L} \gamma^{\mu} d_{L}^{i}\right) \\
J_{W^{-}}^{\mu, i} & =\frac{1}{\sqrt{2}}\left(\bar{\ell}^{i}{ }_{L} \gamma^{\mu} \nu_{L}^{i}+\bar{d}^{i}{ }_{L} \gamma^{\mu} u_{L}^{i}\right)
\end{aligned}
$$

and

$$
\begin{aligned}
J_{Z}^{\mu, i} & =\frac{1}{c_{w}}\left\{\bar{\nu}_{L}^{i} \gamma^{\mu}\left(\frac{1}{2}\right) \nu_{L}^{i}\right. \\
& +\bar{\ell}_{L}^{i} \gamma^{\mu}\left(-\frac{1}{2}+s_{w}^{2}\right) \ell_{L}^{i}+\bar{\ell}_{R}^{i} \gamma^{\mu}\left(s_{w}^{2}\right) \ell_{R}^{i} \\
& +\bar{u}_{L}^{i} \gamma^{\mu}\left(\frac{1}{2}-\frac{2}{3} s_{w}^{2}\right) u_{L}^{i}+\bar{u}_{R}^{i} \gamma^{\mu}\left(-\frac{2}{3} s_{w}^{2}\right) u_{R}^{i} \\
& \left.+\bar{d}_{L}^{i} \gamma^{\mu}\left(-\frac{1}{2}+\frac{1}{3} s_{w}^{2}\right) d_{L}^{i}+\bar{d}_{R}^{i} \gamma^{\mu}\left(\frac{1}{3} s_{w}^{2}\right) d_{R}^{i}\right\}
\end{aligned}
$$

The terms in the electromagnetic current, are also diagonal and do not distinguish between left and right handed particles:

$$
J_{E M}^{\mu, i}=\bar{\ell}^{i} \gamma^{\mu}(-1) \ell^{i}+\bar{u}^{i} \gamma^{\mu}\left(+\frac{2}{3}\right) u^{i}+\bar{d}^{i} \gamma^{\mu}\left(-\frac{1}{3}\right) d^{i}
$$

\section{Fermion masses}

The fermions also acquire mass by coupling to the Higgs field. Since we are specifically interested in understanding top-quark decay, we will restrict the discussion of quark masses to the case of one generation. A complete treatment of quark masses in the case of 
more than one generation must include a description of the weak mixing of quark flavors. However, this phenomenon is not of primary relevance to this analysis.

We can write gauge-invariant Lagrangian describing the Yukawa coupling of the leptons

$$
\Delta \mathcal{L}_{\text {Yukawa }}=-\lambda_{\ell^{i}} \bar{E}_{L}^{i} \phi \ell_{R}^{i}+\text { H.c. }
$$

and the quarks

$$
\Delta \mathcal{L}_{\text {Yukawa }}=-\lambda_{d^{i}} \bar{Q}_{L}^{i} \phi d_{R}^{i}-\lambda_{u^{i}} \epsilon^{a b}\left(\bar{Q}_{L}^{i}\right)_{a}\left(\phi_{b}^{\dagger}\right)_{b} u_{R}^{j}+\text { H.c. }
$$

Here $i$ indexes the generation of the quarks and leptons, and is not summed over. The form of Equations 1.44 and 1.45 differ because right-handed neutrinos are not included in the Standard Model. If we evaluate these terms at the vacuum state, (1.13) we can identify the mass terms; Equation (1.45) becomes

$$
\Delta \mathcal{L}_{\text {Yukawa }}=-\frac{1}{\sqrt{2}} \lambda_{d^{i}} v \bar{d}_{L}^{i} d_{R}^{i}-\frac{1}{\sqrt{2}} \lambda_{u^{i}} v \bar{u}_{L}^{i} u_{R}^{i}+\text { H.c. }
$$

and Equation (1.44) becomes

$$
\Delta \mathcal{L}_{\text {Yukawa }}=-\frac{1}{\sqrt{2}} \lambda_{\ell^{i}} v \bar{\ell}_{L}^{i} \ell_{R}^{i}+\text { H.c. }
$$

Therefore, charged leptons have mass

$$
m_{\ell^{i}}=\frac{1}{\sqrt{2}} \lambda_{\ell^{i}} v
$$

and up- and down-type quarks have mass

$$
m_{u^{i}}=\frac{1}{\sqrt{2}} \lambda_{u^{i}} v, \quad m_{d^{i}}=\frac{1}{\sqrt{2}} \lambda_{d^{i}} v
$$

The Yukawa coupling constants $\lambda_{\ell^{i}}, \lambda_{u^{i}}$ and $\lambda_{d^{i}}$ are inputs to the theory, and are chosen in accordance with the masses of the observed quarks and leptons. 


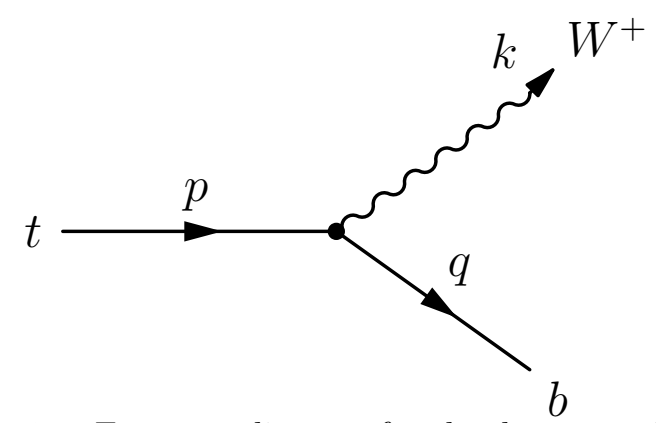

Figure 1.1: Feynman diagram for the decay $t \rightarrow W^{+} b$.

\subsection{Top-quark decay}

According to the Standard Model, the top quark decays almost exclusively via $t \rightarrow$ $W b .^{2}$ The tree-level Feynman diagram for this process is shown in Figure 1.1; we can write the Lorentz-invariant amplitude according to the expression of the weak chargedcurrent (1.41)

$$
i \mathcal{M}=\frac{i g}{\sqrt{2}} \bar{t}(p) \gamma^{\mu}\left(\frac{1-\gamma^{5}}{2}\right) b(q) \epsilon_{\mu}^{*}(k) .
$$

After squaring this amplitude and averaging over the top and bottom polarization states, we find

$$
\frac{1}{2} \sum_{\text {spins }}|\mathcal{M}|^{2}=\frac{g^{2}}{2}\left[q^{\mu} p^{\nu}+q^{\nu} p^{\mu}-g^{\mu \nu} q \cdot p\right] \sum_{\text {polarizations }} \epsilon_{\mu}^{*}(k) \epsilon_{\nu}(k) .
$$

Since $m_{b} / m_{W}<m_{b} / m_{t}<<1$ we can, to a good approximation, treat the $b$ quark as massless. In this limit, energy-momentum conservation gives:

$$
\begin{gathered}
2 p \cdot q=2 p \cdot k=m_{t}^{2}-m_{W}^{2} \\
2 k \cdot p=m_{t}^{2}+m_{W}^{2} .
\end{gathered}
$$

The sum over polarizations in Equation (1.51) depends on the form of the $W^{+}$propagator, and the $W^{+}$propagator depends upon the choice of gauge. The $R_{\xi}$ class of gauges is parametrized by the real, finite constant $\xi$. The $W^{+}$boson propagator in an $R_{\xi}$ gauge

\footnotetext{
2 The decay modes $t \rightarrow W s$ and $t \rightarrow W d$ are also allowed. However, they are suppressed by factors of $\left|V_{t s}\right|^{2} /\left|V_{t b}\right|^{2} \approx 10^{-3}$ and $\left|V_{t d}\right|^{2} /\left|V_{t b}\right|^{2} \approx 5 \times 10^{-4}$, where $V_{i j}$ is the Cabibbo-Kobayashi-Maskawa (CKM) weak-mixing matrix. [21, 17] We will proceed assuming the CKM matrix element $V_{t b}$ is unity.
} 
is

$$
\left\langle W_{\mu}^{+}(k) W_{\nu}^{+}(-k)\right\rangle=\frac{-i}{k^{2}-m_{W}^{2}}\left[g_{\mu \nu}-\frac{k_{\mu} k_{\nu}}{k^{2}-\xi m_{W}^{2}}(1-\xi)\right],
$$

and the propagator for $\phi^{+}$, the Goldstone boson associated with the $W^{+}$boson after EWSB, is

$$
\left\langle\phi^{+}(k) \phi^{+}(-k)\right\rangle=\frac{i}{k^{2}-\xi m_{W}^{2}} .
$$

Note that the parameter $\xi$ is an arbitrary, unphysical parameter. As such, physicallyobservable quantities cannot depend on the choice of the parameter $\xi$ (i.e. the choice of gauge).

In the unitary gauge, (i.e. the limit $\xi \rightarrow \infty$ ) the Goldstone boson propagator vanishes (by definition), and the gauge boson propagator is

$$
\left\langle W_{\mu}^{+}(k) W_{\nu}^{+}(k)\right\rangle=\frac{-i}{k^{2}-m_{W}^{2}}\left(g_{\mu \nu}-\frac{k_{\mu} k_{\nu}}{m_{W}^{2}}\right) .
$$

Notice that this propagator includes one longitudinal polarization state (the term proportional to $k_{\mu} k_{\nu}$ ) and the two transverse polarization states appropriate for an on-shell massive vector particle. The sum over physical polarization states of the $W^{+}$is then

$$
\left[\sum \epsilon_{\mu}^{*}(k) \epsilon_{\nu}(k)\right]_{\lim \xi \rightarrow \infty}=g_{\mu \nu}-\frac{k_{\mu} k_{\nu}}{m_{W}^{2}}
$$

Inserting this expression into Equation (1.51), we find

$$
\frac{1}{2} \sum_{\text {spins }}|\mathcal{M}|^{2}=\frac{g^{2}}{4} \frac{m_{t}^{4}}{m_{W}^{2}}\left(1-\frac{m_{W}^{2}}{m_{t}^{2}}\right)\left(1+2 \frac{m_{W}^{2}}{m_{t}^{2}}\right) .
$$

This gives the Standard Model prediction for the top quark width,

$$
\Gamma=\frac{g^{2}}{64 \pi} \frac{m_{t}^{3}}{m_{W}^{2}}\left(1-\frac{m_{W}^{2}}{m_{t}^{2}}\right)^{2}\left(1+2 \frac{m_{W}^{2}}{m_{t}^{2}}\right) .
$$

The top width (1.59) is a physically-observable quantity, and therefore must be gaugeinvariant. However, if we work in the Feynman-'t Hooft gauge, (i.e. $\xi=1$ ) we see that 


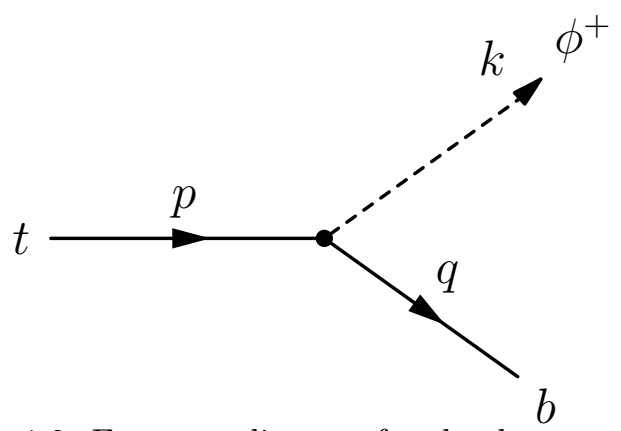

Figure 1.2: Feynman diagram for the decay $t \rightarrow \phi^{+} b$.

the $W^{+}$contributes only the transverse polarization states to the width

$$
\frac{1}{2} \sum_{\text {spins }}|\mathcal{M}|^{2}=\frac{g^{2}}{2}(2 p \cdot q)=\frac{g^{2}}{2}\left(m_{t}^{2}-m_{W}^{2}\right),
$$

a result inconsistent with Equation (1.58). Gauge invariance is saved when we include the process $t \rightarrow \phi^{+} b$, shown in Figure 1.2. In the Feynman-'t Hooft gauge, the Goldstone boson contributes the missing longitudinal polarization terms to the top width

$$
\begin{aligned}
i \mathcal{M}\left(t \rightarrow \phi^{+} b\right) & =\lambda_{t}^{2} \bar{b}(q)\left(\frac{1+\gamma^{5}}{2}\right) t(p), \\
\frac{1}{2} \sum_{\text {spins }}\left|\mathcal{M}\left(t \rightarrow \phi^{+} b\right)\right|^{2} & =\lambda_{t}^{2} p \cdot q \\
& =\frac{g^{2}}{4} \frac{m_{t}^{2}}{m_{W}^{2}}\left(m_{t}^{2}-m_{W}^{2}\right) .
\end{aligned}
$$

We have shown that while the top quark width is gauge-invariant, the distinction between the longitudinal polarization state of the $W^{+}$or the Goldstone boson $\phi^{+}$depends upon an arbitrary choice of gauge.

\subsubsection{The fraction of longitudinally-polarized $W$ s from top-quark decays}

Using the results of the previous section, we can calculate the SM prediction of the fraction of longitudinally-polarized $W$ s produced in top decays,

$$
F_{0} \equiv \frac{\Gamma\left(\lambda_{W}=0\right)}{\Gamma\left(\lambda_{W}=-1\right)+\Gamma\left(\lambda_{W}=0\right)+\Gamma\left(\lambda_{W}=+1\right)} .
$$


Because of the $V-A$ nature of the SM weak interaction, only the terms $\Gamma\left(\lambda_{W}=-1\right)$ and $\Gamma\left(\lambda_{W}=0\right)$ appear in the denominator. The final result is

$$
F_{0}=\left(1+2\left(\frac{m_{W}}{m_{t}}\right)^{2}\right)^{-1}
$$

For $m_{t}=178 \mathrm{GeV}$, the $\mathrm{SM}$ prediction is $F_{0} \simeq 0.71$.

\subsubsection{Non-universal $t W b$ gauge couplings}

So far, we have worked under the assumption that the $V-A$ structure of the chargedcurrent interaction is universal for all quark generations. However, because the top quark is heavy, it is possible that new physics may be manifest at the $t W b$ vertex in the form of non-universal gauge couplings [22]. The most general Lagrangian for the $t W b$ vertex which describes such non-universal couplings is [23]

$$
\begin{aligned}
\mathcal{L}= & \frac{g}{\sqrt{2}}\left[W_{\mu}^{-} \bar{b} \gamma^{\mu}\left(f_{1}^{L} P_{-}+f_{1}^{R} P_{+}\right) t-\frac{1}{M_{W}} \partial_{\nu} W_{\mu}^{-} \bar{b} \sigma^{\mu \nu}\left(f_{2}^{L} P_{-}+f_{2}^{R} P_{+}\right) t\right] \\
& +\frac{g}{\sqrt{2}}\left[W_{\mu}^{+} \bar{t} \gamma^{\mu}\left(f_{1}^{L^{*}} P_{-}+f_{1}^{R^{*}} P_{+}\right) b-\frac{1}{M_{W}} \partial_{\nu} W_{\mu}^{+} \bar{t} \sigma^{\mu \nu}\left(f_{2}^{R^{*}} P_{-}+f_{2}^{L^{*}} P_{+}\right) b\right] .
\end{aligned}
$$

The form factors $f_{1}^{L}$ and $f_{1}^{R}$ parametrize the strength of left- and right-handed charged current interactions. The strength of an anomalous weak magnetic interaction is parametrized by the form factors $f_{2}^{L}$ and $f_{2}^{R}$. At tree level in the SM the form factors are $f_{1}^{L}=1$ and $f_{1}^{R}=f_{2}^{L}=f_{2}^{R}=0$.

The polarization of the $W$ in the $t \rightarrow W b$ decay probes the values of the form factors $f_{1}^{L}$ and $f_{2}^{L}$. The amplitudes squared for the possible $W$ polarizations are (apart from a 
common factor $\left.g^{2} E_{b} m_{t}\right)[23]$

$$
\begin{aligned}
\left|\mathcal{M}\left(\lambda_{W}=-\right)\right|^{2} & =\left|f_{1}^{L}+\frac{m_{t}}{M_{W}} f_{2}^{R}\right|^{2}, \\
\left|\mathcal{M}\left(\lambda_{W}=+\right)\right|^{2} & =\left|f_{1}^{R}+\frac{m_{t}}{M_{W}} f_{2}^{L}\right|^{2}, \\
\left|\mathcal{M}\left(\lambda_{W}=0\right)\right|^{2} & =\frac{1}{2}\left|\frac{m_{t}}{M_{W}} f_{1}^{L}+f_{2}^{R}\right|^{2}+\frac{1}{2}\left|\frac{m_{t}}{M_{W}} f_{1}^{R}+f_{2}^{L}\right|^{2} .
\end{aligned}
$$

While there is direct experimental information regarding $f_{1}^{R}$ (see Section 2.3), this form factor is already severely bounded (albeit indirectly) by the observed $b \rightarrow s \gamma$ rate $[24,25]$,

$$
-0.037<f_{1}^{R}<0.0015 @ 95 \% \text { CL. }
$$

We proceed under the assumption that there are no non-universal couplings at the $t W b$ vertex, and set out to simply measure $F_{0}$, thereby testing the consistency of the theory. 


\section{CHAPTER 2}

\section{Top production and decay}

In this chapter we discuss top-quark pair production at the Tevatron. We describe the experimental signatures of $t \bar{t}$ decay and present a brief description of methods used to isolate $t \bar{t}$-enriched samples in the data. We discuss several approaches for measuring $F_{0}$ in these data; we also present the results of some previous measurements of this quantity from the DØ and CDF experiments. Finally, we describe the methods used in this analysis to measure $F_{0}$.

\subsection{Top-quark production at the Tevatron}

The strong interaction is primarily responsible for producing top quarks in $p \bar{p}$ collisions at the Tevatron. The weak interaction can produce single top quarks, however the rate of weak production is expected to be $\sim 1 / 3$ that of strong production. In addition, backgrounds from SM processes (e.g. Wgg production) are large, making it difficult to isolate single-top events. The strong interaction produces top quarks in pairs; many SM processes do mimic the signatures for $t \bar{t}$ decays, however the problem of isolating topquark pairs is much more tractable, and great effort has been devoted to understanding and reducing these backgrounds.

The dynamics of the interaction of gluons and quarks is described by the QCD terms 

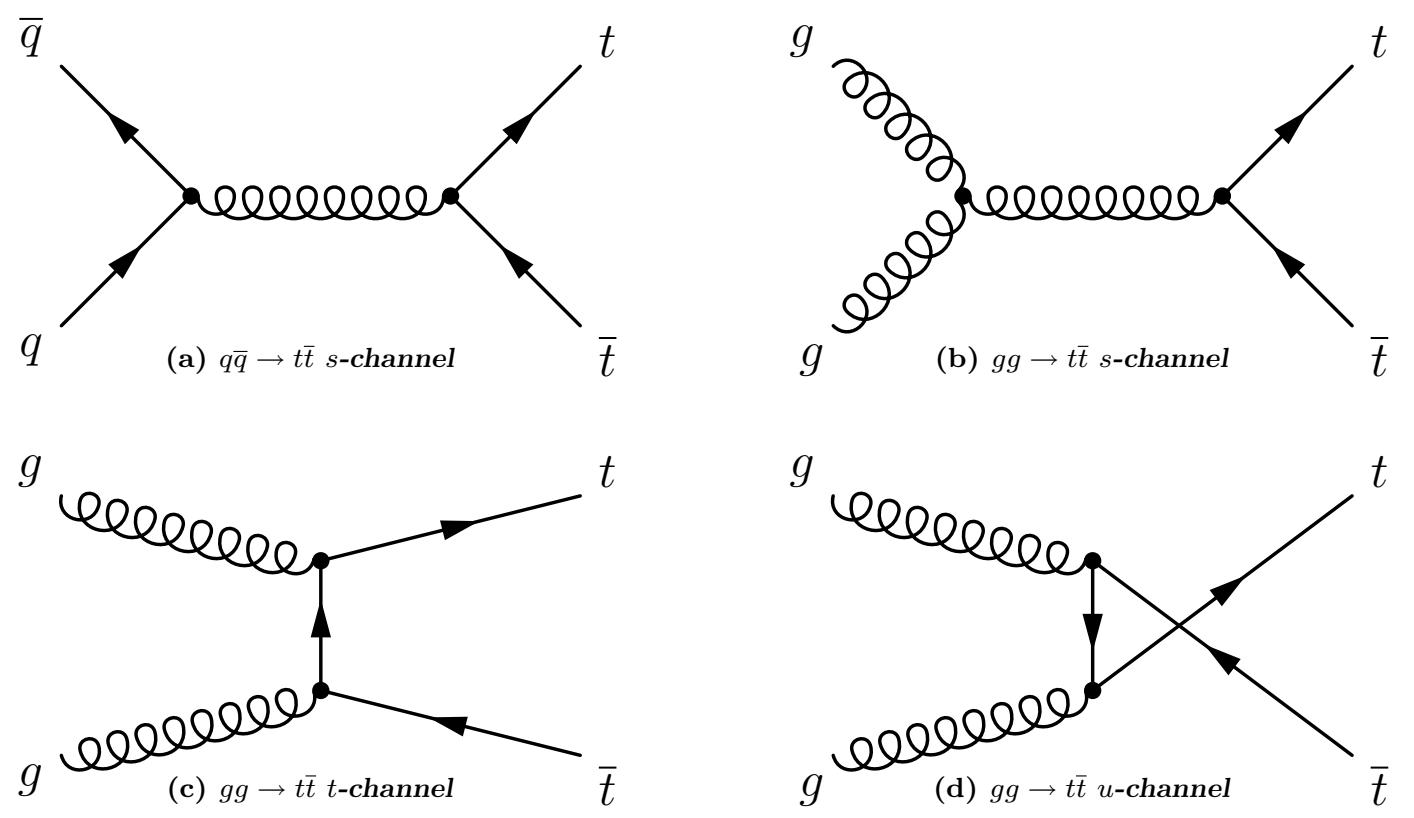

Figure 2.1: Feynman diagrams of the leading order $t \bar{t}$ pair production processes.

of the SM Lagrangian

$$
\Delta \mathcal{L}_{\mathrm{QCD}}=-\frac{1}{2} g_{s} \bar{q} \gamma^{\mu} \lambda^{a} G_{\mu}^{a} q-\frac{1}{4} G_{\mu \nu}^{a} G^{a \mu \nu}
$$

The quarks appear as $\mathrm{SU}(3)$ triplets $q$, and $G_{\mu}^{a=1, \ldots, 8}$ are the eight gluon fields associated with the eight generators of $\mathrm{SU}(3)$. The kinetic terms $G^{a}{ }_{\mu \nu} G^{a \mu \nu}$ describe gluon-gluon interactions,

$$
G_{\mu \nu}^{a}=\partial_{\mu} G^{a}{ }_{\nu}-\partial_{\nu} G^{a}{ }_{\mu}-g_{3} f^{a b c} G_{\mu}^{b} G_{\nu}^{c}
$$

where $f^{a b c}$ are the structure constants for $\mathrm{SU}(3)$.

The leading order Feynman diagrams for the production of $t \bar{t}$ pairs in $p \bar{p}$ collisions are shown in Figure 2.1. Of these processes, the $q \bar{q}$ initial state is expected to produce $85 \%$ of $t \bar{t}$ pairs at the Tevatron; the $g g$ initial state is expected to contribute the remaining 15\%. From these diagrams one can calculate the Lorentz invariant amplitudes $\mathcal{M}$ for these processes. The differential cross section for a process $p_{i} p_{j} \rightarrow p_{1} p_{2}$ is

$$
\frac{d \sigma_{i j}}{d \Omega_{C M}}=\frac{\left|p_{1}\right|}{32 \pi^{2} E_{i} E_{j} E_{C M}\left|v_{i}-v_{j}\right|}\left|\mathcal{M}\left(p_{i} p_{j} \rightarrow p_{1} p_{2}\right)\right|^{2}
$$




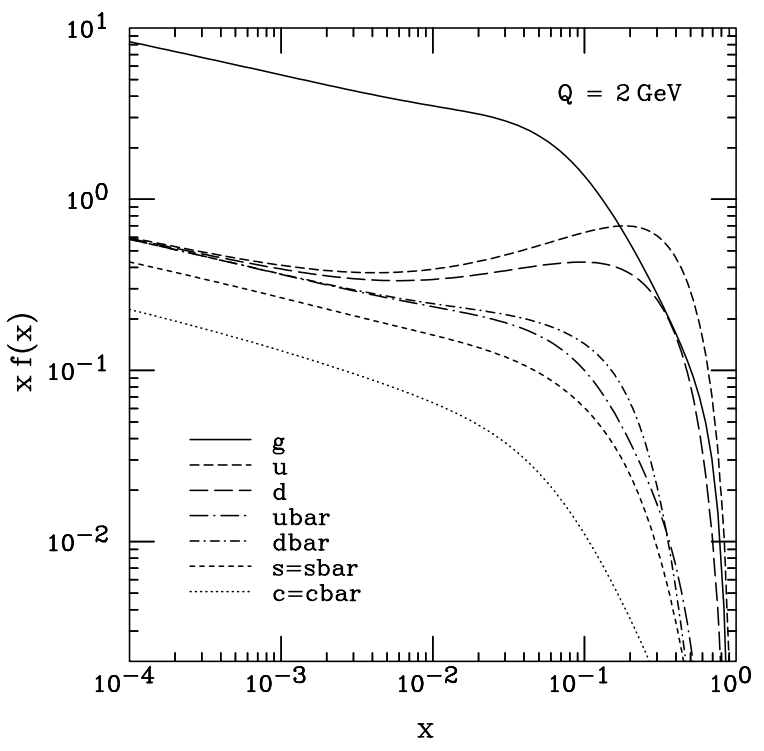

(a)

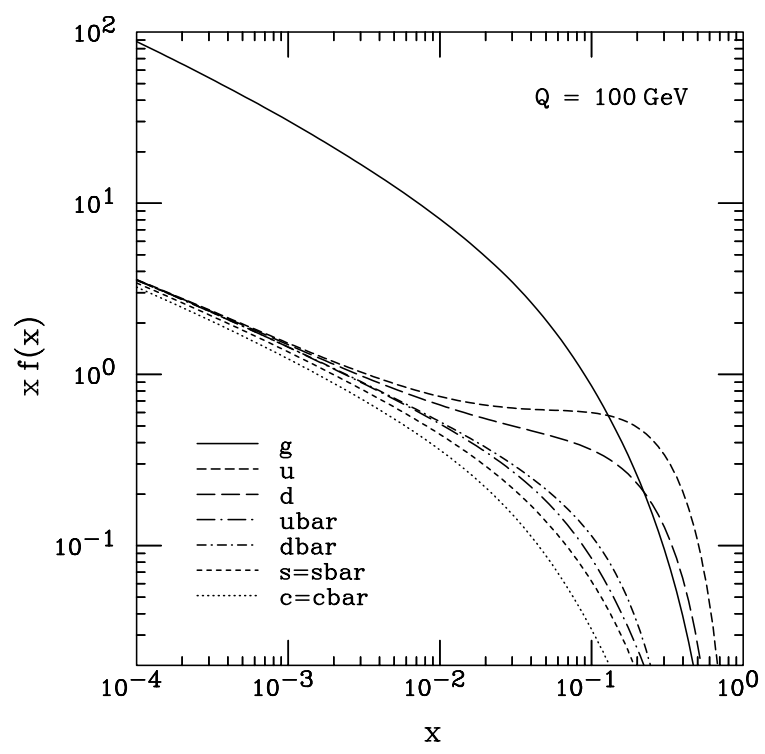

(b)

Figure 2.2: Parton distribution functions at (a) $Q^{2}=2 \mathrm{GeV}$ and (b) $Q^{2}=100 \mathrm{GeV}[26]$.

Protons (and antiprotons) are not point-like objects: they are assemblages of real quarks, surrounded by a sea of virtual quarks and bound by gluons. At sufficiently high energies, collisions between the proton constituents (called partons) can be regarded as interactions between free particles. In this limit, the total cross section for a given process is approximated by summing over all possible parton interactions:

$$
\sigma(p \bar{p} \rightarrow t \bar{t})=\sum_{i, j} \int d x_{i} d x_{j} F_{p}\left(x_{i}, Q^{2}\right) F_{\bar{p}}\left(x_{j}, Q^{2}\right) \sigma_{i j}\left(Q^{2}, m_{t}\right) .
$$

The factorization and renormalization scale $Q^{2}$ is an arbitrary parameter with dimensions of energy, which is introduced in the renormalization procedure. The functions $F_{p}\left(x_{i}, Q^{2}\right)$ and $F_{\bar{p}}\left(x_{i}, Q^{2}\right)$ are the parton distribution functions. The product $F_{p}\left(x_{i}, Q^{2}\right) F_{\bar{p}}\left(x_{j}, Q^{2}\right)$ is the joint probability density of parton $i$, (within the proton) with momentum $x_{i} P$, interacting with parton $j$, (within the antiproton) with momentum $-x_{j} P$. Here $P(-P)$ is the magnitude of the proton (antiproton) momentum in the center of mass frame. The parton distribution functions are determined empirically: parametrizations of these functions are derived from fits to data of deep inelastic scattering experiments at low 
$Q^{2}[26]$. These parametrizations are scaled to appropriately large values $Q^{2}$ using the DGLAP equations [27, 28, 29]. Figure 2.2 shows these parametrizations at different values of $Q^{2}$.

For a center of mass energy $\sqrt{s}=1.96 \mathrm{TeV}$ and a top-quark mass $m_{t}=175 \mathrm{GeV}$, the total theoretical cross section for $t \bar{t}$ production is [30]

$$
\sigma(p \bar{p} \rightarrow t \bar{t})=6.7_{-0.9}^{+0.7} \mathrm{pb}
$$

The CDF collaboration has measured the $t \bar{t}$ pair-production cross section using data from run II of the Tevatron. Figure 2.3 summarizes the results of these analyses, which are in good agreement with the theoretical expectation. The consistency of the measured cross section with the theoretical prediction implies that we have a good understanding of the background content of these samples, and that we have indeed isolated $t \bar{t}$ events in these data. Figure 2.4 summarizes the recent measurements of the $t \bar{t}$ production crosssection by the $D \varnothing$ collaboration; these are also in good agreement with the theoretical expectation.

\subsection{Experimental signatures of $t \bar{t}$ decay}

As discussed in Section 1.2, the SM predicts that the top quark decays almost exclusively via the process $t \rightarrow W b$. It's convenient to classify the final states of $t \bar{t}$ events according to the decay of the $W$ bosons. In this analysis we consider only the final states where one or both $W$ bosons decay leptonically. We discuss these samples in greater detail in Chapter 4.

- The dilepton final state: Both $W$ 's decay to a charged lepton and a neutrino. We nominally consider only $W$ decays to an electron or a muon, giving this channel has a branching ratio $\mathcal{B}=4 / 81$. However, the effective ratio is slightly larger, 


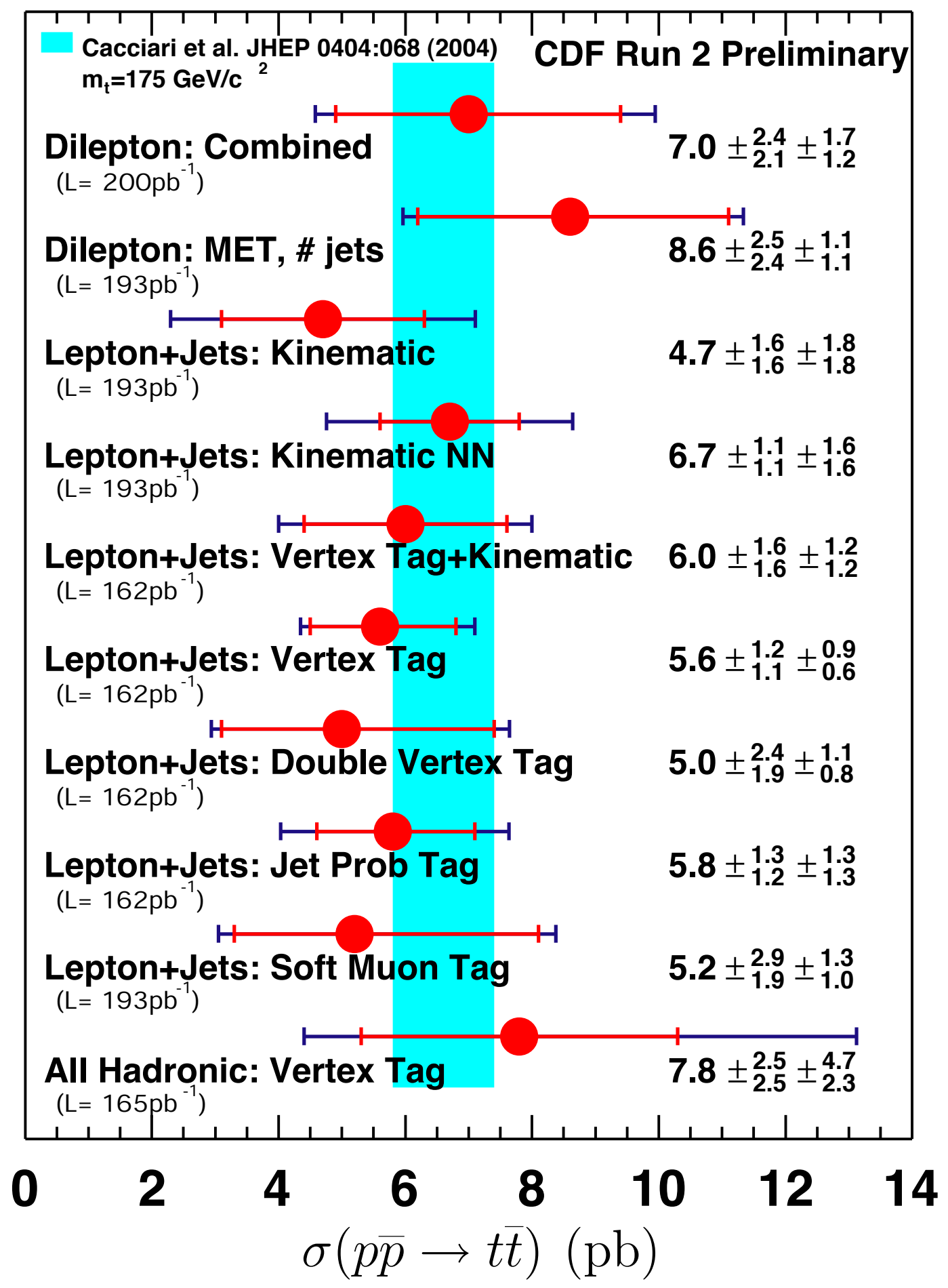

Figure 2.3: A summary of the top-quark pair production cross section measurements performed by the CDF collaboration in run II. The red and blue bars indicate statistical and systematic errors, respectively. Note that the samples used in the dilepton and the lepton+jets measurements are non-disjoint, therefore the dilepton and the lepton+jets results are correlated. 


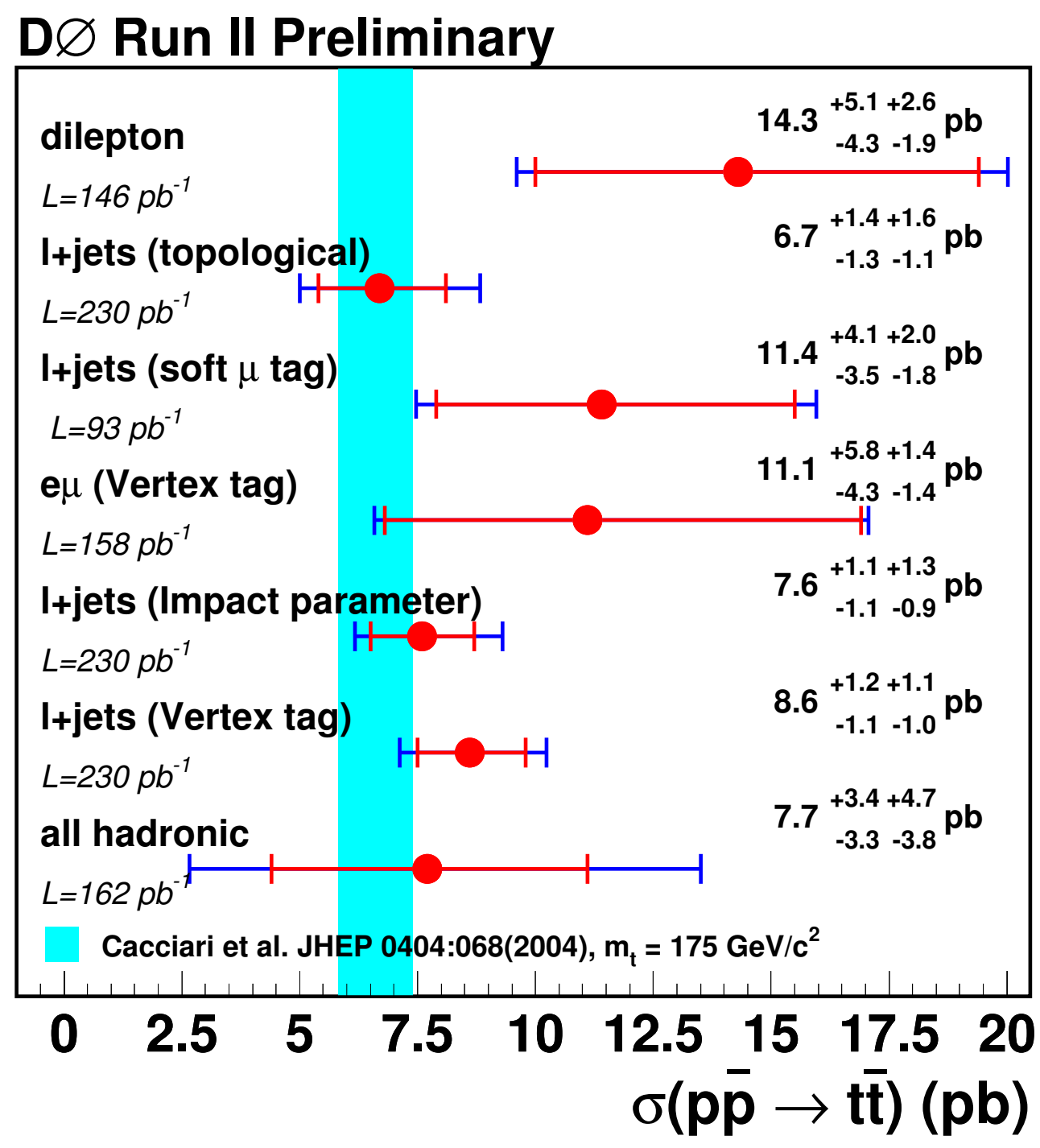

Figure 2.4: A summary of the top-quark pair production cross section measurements performed by the D $\varnothing$ collaboration in run II. The red and blue bars indicate statistical and systematic errors, respectively. 
since $W$ decay to a tau with subsequent decay to an electron or muon and two neutrinos contributes as well. This channel has the smallest branching ratio, and a low background rate. The experimental signature for this channel consists of two energetic, oppositely-charged central leptons, two or more central jets, and "missing energy" in the transverse plane, presumably due to the unobserved neutrinos.

- The lepton+jets final state: one $W$ decays to a charged lepton and a neutrino, the other decays to quarks (i.e. $W \rightarrow c \bar{s}, W \rightarrow u \bar{d}$ ). This channel has a nominal branching ratio $\mathcal{B}=12 / 27$ (again, the actual branching ratio is slightly larger due to the contribution of leptonic tau decays). The lepton+jets channel suffers from a larger background rate than that of the dilepton channel; these backgrounds can be reduced by requiring that at least one jet have a displaced secondary vertex, indicating it is consistent with the decay of a long-lived b-hadron. The experimental signature for this channel consists of one energetic charged central lepton, three or more energetic central jets (at least one of which must have a displaced secondary vertex " $b$-tag"), and missing energy (again, presumably due to the unobserved neutrino). Since not all partons are manifest as jets in the detector, (e.g. two collinear partons can "merge" and appear as a single jet; a high-rapidity parton could be outside the fiducial region of the detector) we improve the overall event acceptance by requiring three or more jets.

- The all-hadronic final state: both $W^{\prime}$ s decay hadronically. Consequently, this channel has the largest branching fraction, $\mathcal{B}=4 / 9$, and because of QCD processes which can closely mimic this channel's experimental signature, it has a very large background rate. The experimental signature for this channel nominally consists of six energetic jets. As with the lepton+jets channel, backgrounds can be reduced by 


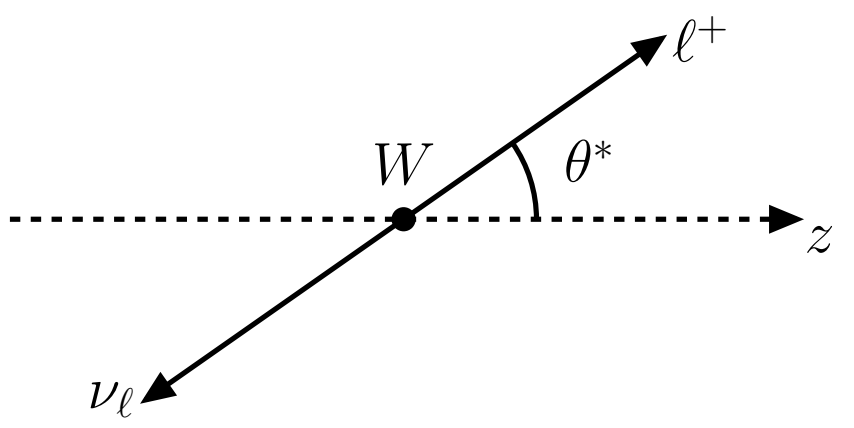

Figure 2.5: The angle between the charged-lepton three-momentum and the $W$ boost vector in the $W$ rest frame.

requiring that one or more jets have a $b$-tag.

\subsection{Kinematic variables with helicity information}

The distributions of several kinematic variables hold information from which we can deduce $F_{0}$, the fraction of longitudinally polarized $W$ bosons produced in top-quark decays. The canonical variable for such an analysis is the polar angle $\theta^{*}$. Shown in Figure $2.3, \theta^{*}$ is the angle between the charged lepton three-momentum and the $z$-axis in the rest frame of the $W$, where the $z$-axis is along the $W$ boost direction in the rest frame of the top quark.

The helicity amplitudes for the process $W^{+} \rightarrow \ell^{+} \nu_{\ell}$ are well known. Ignoring the common factor $g m_{W}$, the helicity amplitudes for the decay of left-handed, longitudinal and right-handed $W$ bosons are ${ }^{1}$

$$
\begin{aligned}
\text { left }- \text { handed }:\left(\lambda_{W}=-1\right) & =-e^{-i \phi_{e}^{*}}\left(\frac{1-\cos \theta_{\ell}^{*}}{2}\right), \\
\text { longitudinal }:\left(\lambda_{W}=0\right) & =-\frac{\sin \theta_{\ell}^{*}}{\sqrt{2}}, \\
\text { right }- \text { handed }:\left(\lambda_{W}=+1\right) & =-e^{i \phi_{\ell}^{*}}\left(\frac{1+\cos \theta_{\ell}^{*}}{2}\right),
\end{aligned}
$$

where $\phi_{\ell}^{*}$ is the azimuthal angle of the charged lepton in the rest frame of $W^{+}$. In terms

\footnotetext{
1 The helicity amplitudes $\left(\lambda_{W}\right)$ for the process $W^{-} \rightarrow \ell^{-} \overline{\nu_{\ell}}$ can be obtained from Equation (2.6) by replacing $\theta_{\ell}^{*}$ with $\pi-\theta_{\ell}^{*}$ and $\phi_{\ell}^{*}$ with $\pi+\phi_{\ell}^{*}$.
} 


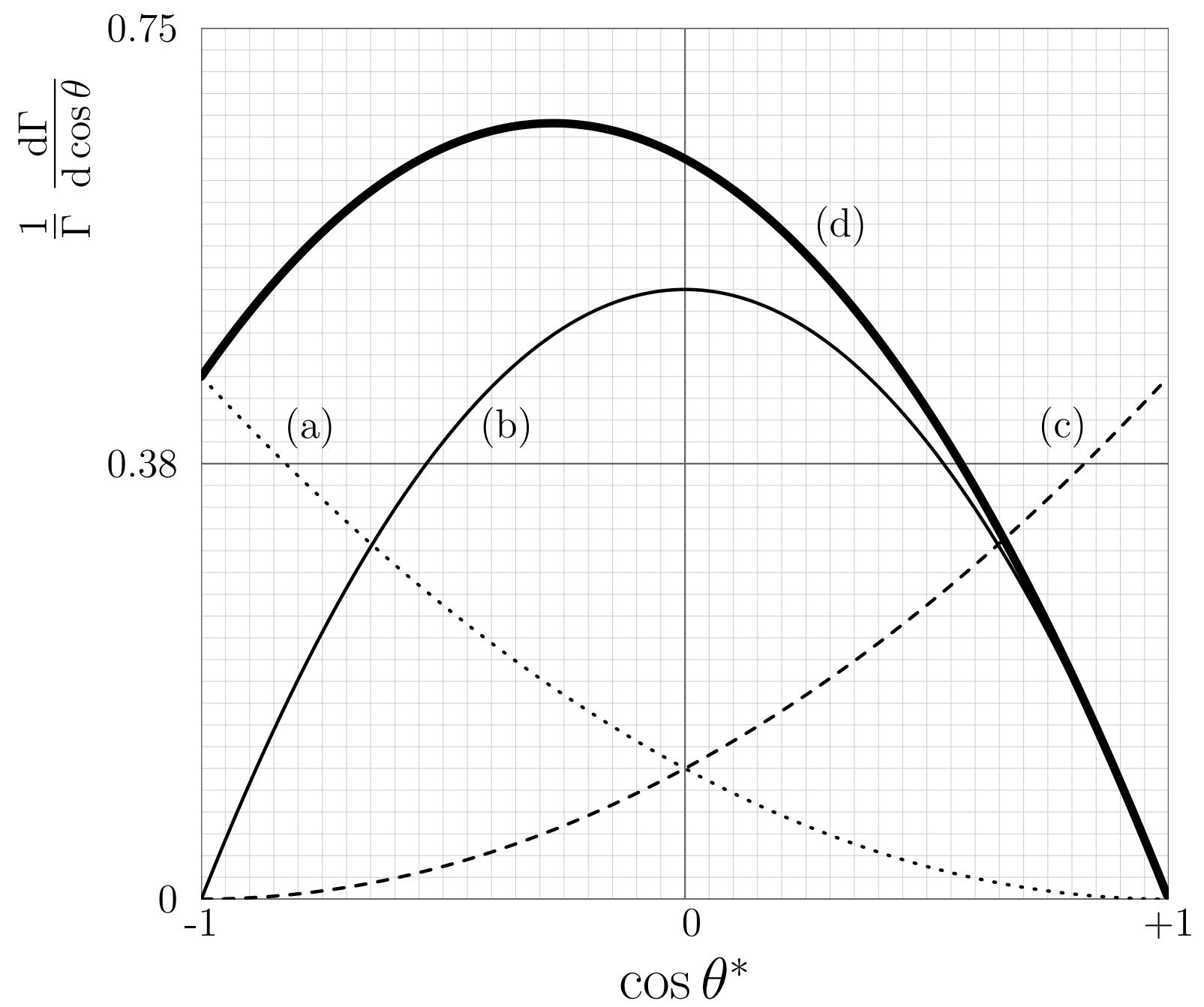

Figure 2.6: Differential decay rates for the process $W_{\lambda} \rightarrow \ell \nu_{\ell}$. The curves labeled (a), (b) and (c) are the distributions for $\lambda_{W}=-1,0$, and +1 , respectively. The curve labeled (d) is the distribution predicted by the Standard Model (i.e. a 70\% longitudinal 30\% left-handed admixture).

of these amplitudes, the differential decay rate is

$$
\frac{1}{\Gamma} \frac{\mathrm{d} \Gamma}{\mathrm{d} \cos \theta^{*}}=\frac{3}{8} F_{-}\left(1-\cos \theta^{*}\right)^{2}+\frac{3}{4} F_{0}\left(1-\cos ^{2} \theta^{*}\right)+\frac{3}{8} F_{+}\left(1+\cos \theta^{*}\right)^{2} .
$$

Figure 2.6 shows the angular distribution of charged leptons whose parent is a $W$ with helicity (a) $\lambda_{W}=-1$, (b) $\lambda_{W}=0$ or (c) $\lambda_{W}=+1$.

A direct approach to measuring $F_{0}$ would be to examine the distribution of reconstructed $\cos \theta^{*}$ from top-enriched data and fit that observed distribution to an expression similar in form to Equation (2.7), i.e. a likelihood function parametrized by $F_{0}$. How- 
ever, we are limited in our knowledge of how each event in the data truly proceeded; we cannot distinguish on an event-by-event basis between signal and background, nor can we determine in this basis if we have properly associated observed jets with the correct pre-hadronization parton. So while we can extract useful information about $F_{0}$ from this distribution, we can expect significant statistical and systematic uncertainties due to the inevitable misidentification and misreconstruction of events.

Another approach is to approximate $\cos \theta^{*}$ using the invariant mass of the bottom quark and the charged lepton. This relationship is simply

$$
\begin{aligned}
\cos \theta^{*} & =\frac{E_{\ell} E_{b}-p_{\ell} \cdot p_{b}}{\left|\mathbf{p}_{\ell}\right|\left|\mathbf{p}_{b}\right|} \\
& \simeq 1-\frac{p_{\ell} \cdot p_{b}}{E_{\ell} E_{b}}=1-\frac{2 m_{\ell b}^{2}}{m_{t}^{2}-M_{W}^{2}}
\end{aligned}
$$

Here the energies $E_{\ell}$ and $E_{b}$ are

$$
\begin{array}{ll}
E_{\ell}=\frac{M_{W}^{2}+m_{\ell}^{2}-m_{\nu_{\ell}}^{2}}{2 M_{W}}, & \left|\mathbf{p}_{\ell}\right|=\sqrt{E_{\ell}^{2}-m_{\ell}^{2}}, \\
E_{b}=\frac{m_{t}^{2}-M_{W}^{2}-m_{b}^{2}}{2 M_{W}}, & \left|\mathbf{p}_{b}\right|=\sqrt{E_{b}^{2}-m_{b}^{2}} .
\end{array}
$$

This approach is favorable, since to reconstruct $m_{\ell b}$, one need only match the charged lepton with the appropriate $b$-jet (i.e. the charged lepton and the $b$ quark must share the same $t$ quark parent). In run II, CDF measured $F_{0}$ in the lepton+jets channel using this method [31]. The result is

$$
\begin{aligned}
& F_{0}=0.99_{-0.35}^{+0.29} \text { (stat.) } \pm 0.19 \text { (syst.) } \\
& F_{0}>0.33 @ 95 \% \mathrm{CL}
\end{aligned}
$$


In run I, CDF measured $F_{+}$in the lepton+jets and dilepton channels using this method [32, 33, 34]. The result is

$$
F_{+}<0.18 @ 95 \% \text { CL }
$$

Another approach is the matrix element method, where the complete invariant matrix element for $t \bar{t}$ production and decay is used as a pseudo-likelihood function, parametrized by the top-quark mass and $F_{0}$. This method offers great statistical precision, since it uses maximal kinematic information from each event. It also eliminates a leading source of systematic error, the uncertainty on top mass. Requisite for this method is a detailed understanding of the calorimeter response, expressed in terms a mapping between jet and parton energies, that $\mathrm{CDF}$ in run II has only recently realized. In run I, D $\varnothing$ measured $F_{0}$ in the lepton+jets channel using this method $[35,36]$. The result is

$$
F_{0}=0.56 \pm 0.31 \text { (stat.) } \pm 0.07 \text { (syst.) }
$$

Considering the modest size of DØ's lepton+jets sample in run I, and the relatively large background content of that sample, the precision of this result is impressive.

In this analysis we have chosen to avoid the problem of combinatoric backgrounds due to event misreconstruction by using as an observable the transverse momentum $\left(p_{T}\right)$ of the charged lepton in the laboratory frame. Charged-lepton $p_{T}$ is the best-measured per-event kinematic variable at CDF, and it is a good discriminant of $W$ helicity. In run I CDF measured $F_{0}$ in the lepton+jets and dilepton channels using this method [37, 38]. The run I result is

$$
F_{0}=0.91 \pm 0.37 \text { (stat.) } \pm 0.13 \text { (syst.). }
$$

The distributions of charged lepton $p_{T}$ differ according to the helicity of the parent $W$. Based on the differential decay rates shown in Figure 2.6, we can make the following qual- 


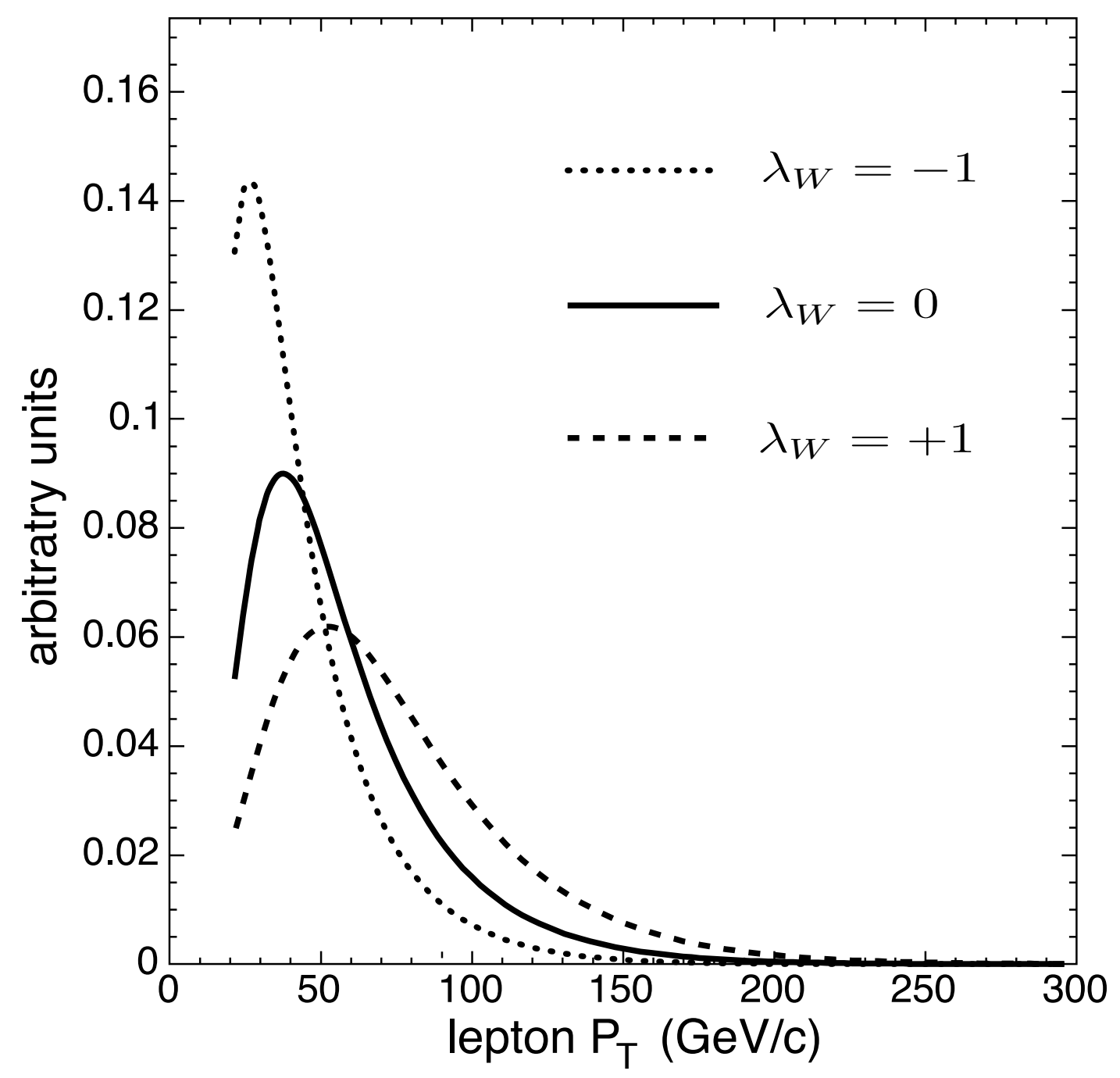

Figure 2.7: Distributions of the transverse momentum of charged leptons from leptonically decaying $W$ bosons in $t \bar{t}$ events, where the $W$-parent of the charged lepton has helicity $\lambda_{W}=-1, \lambda_{W}=0$ or $\lambda_{W}=+1$.

itative argument: left-handed $W$ bosons tend to produce charged leptons with momenta anti-parallel to the boost vector of the parent $W$, giving rise to a "soft" $p_{T}$ distribution in the laboratory frame; longitudinal $W$ bosons tend to produce charged leptons with momenta perpendicular to the boost vector of the parent $W$, giving rise to a less-soft $p_{T}$ distribution; right-handed $W$ bosons tend to produce charged leptons with momenta parallel to the boost vector of the parent $W$, giving rise to a "hard" $p_{T}$ distribution.

Figure 2.7 shows the charged-lepton $p_{T}$ distribution of leptonically decaying $W$ bosons 
in $t \bar{t}$ events, where the $W$-parent has helicity $\lambda_{W}=-1, \lambda_{W}=0$ or $\lambda_{W}=+1$. These distributions are drawn from events generated using a customized version of the HERWIG Monte Carlo (MC) event generator [39] which allows one to simulate $t \bar{t}$ production and decay, where the helicity admixture of either $W$ is fixed by the user. This modified version of HERWIG was developed by Gene Guillian at the University of Michigan [40], and has been vetted by the HERWIG authors [41]. The generated events are passed through version 4.11.1 of the $\mathrm{CDF}$ detector simulation (which models the detector response to these data), and offline reconstruction software (which computes primitive physics objects for later analysis). The event selection requirements of the dilepton are lepton+jets cross section analyses are applied. These are described in detail in Chapter 4. 


\section{CHAPTER 3}

\section{Experimental apparatus}

Since top quarks are so massive, high-energy collisions are needed to produce them. At present only the Tevatron synchrotron at the Fermi National Accelerator Laboratory located in Batavia, Illinois is capable of producing collisions with energies sufficient for direct production of $t \bar{t}$ pairs.

Since the top-quark pair production cross section is so small relative to the total cross section for $p \bar{p}$ collisions at high energy, $\left(\sigma(p \bar{p} \rightarrow t \bar{t}) / \sigma(p \bar{p} \rightarrow\right.$ anything $\left.) \sim 10^{-10}\right)$ many collisions are needed to produce an appreciable sample of $t \bar{t}$ events. In addition, a detector capable of recording detailed event kinematics and a trigger capable of quickly discriminating "interesting" events from overwhelming backgrounds is necessary to study the properties of $t \bar{t}$ production and decay. The Collider Detector at Fermilab (CDF) and its trigger serve this purpose.

The Tevatron [42] and the CDF detector [43, 44] have recently been upgraded for a period of data-taking called run II. The upgraded Tevatron collides protons and antiprotons with a center of mass energy of $1.96 \mathrm{TeV}$. In this analysis we consider run II data taken from July 2001 through September 2003, corresponding to an integrated luminosity of $200 \mathrm{pb}^{-1}$.

In this chapter we describe the Fermilab accelerator complex and the CDF detector 


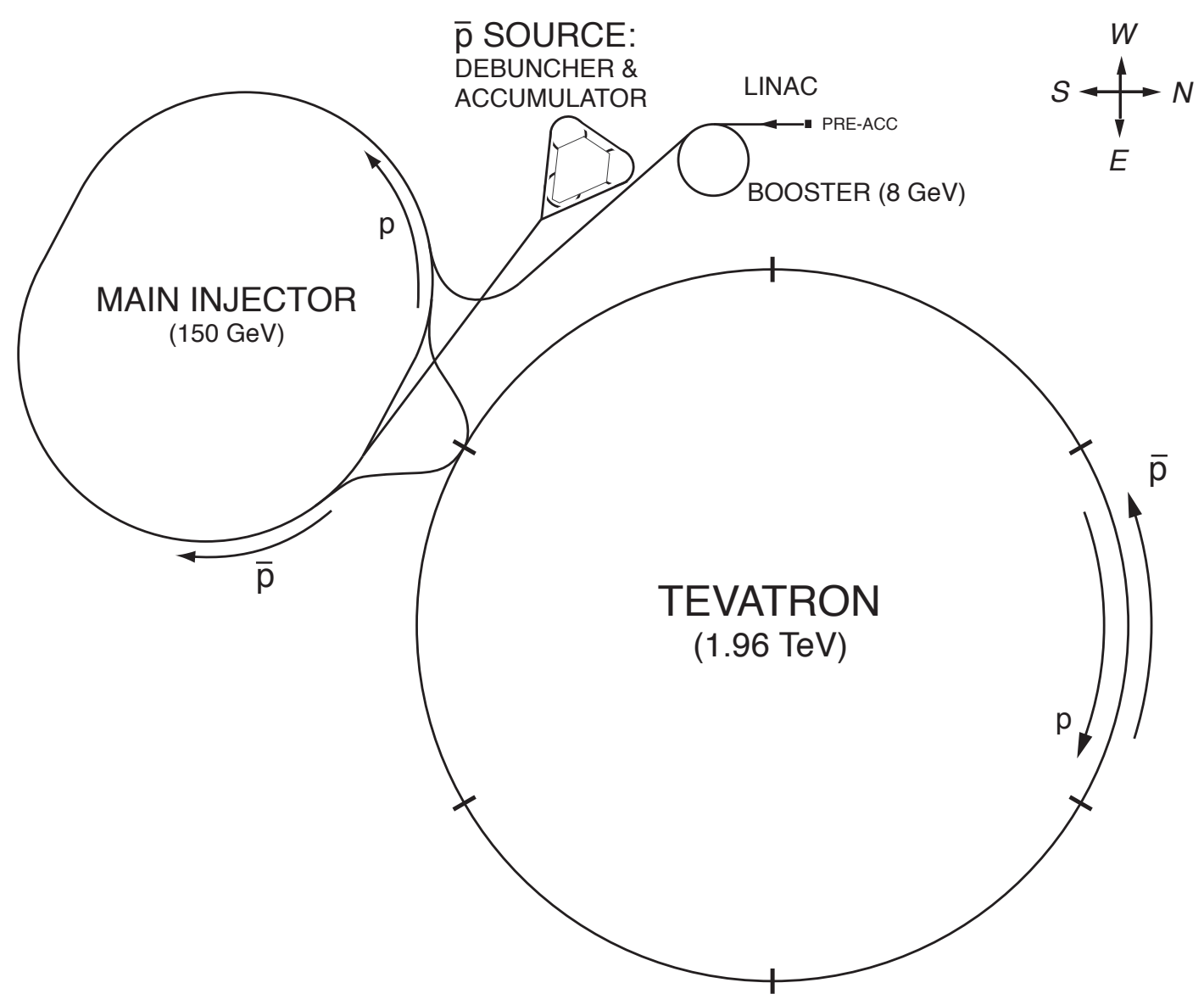

Figure 3.1: A schematic representation of the Fermilab accelerator complex.

during run II.

\subsection{The Fermilab accelerator complex}

The Fermilab accelerator complex consists of a series of eight accelerators, each of increasing final beam energy. The accelerator complex is illustrated in Figure 3.1.

Protons are drawn from $\mathrm{H}^{-}$ions; these ions are accelerated from rest to an energy of $750 \mathrm{keV}$ by a Cockroft-Walton electrostatic generator. The ions are injected into a $150 \mathrm{~m}$ linear RF accelerator (the Linac) which boosts their energy to $400 \mathrm{MeV}$. The $H^{-}$ions then pass through a graphite foil which strips them of their electrons and leaves the bare 
protons.

The protons are injected into the Booster, a synchrotron $474 \mathrm{~m}$ in circumference. Bending magnets guide the protons along the curved path of the Booster while RF cavities accelerate them. The frequency of the accelerating potential and the strength of the magnetic field increase gradually to bring the protons to an energy of $8 \mathrm{GeV}$.

Protons from the Booster are merged into bunches and are sent to the Main Injector, a synchrotron $3 \mathrm{~km}$ in circumference. There, the proton bunches are accelerated to $150 \mathrm{GeV}$. The protons are then injected into the Tevatron, a synchrotron $6.3 \mathrm{~km}$ in circumference, which employs superconducting bending magnets and RF cavities to accelerate the proton bunches to $980 \mathrm{GeV}$.

Some of the protons from the Main Injector are used to produce antiprotons. Protons are accelerated to $120 \mathrm{GeV}$ and are guided to impact a nickel target. This impact produces many particles; antiprotons are produced with an effective efficiency of $10^{-5}$. These antiprotons are isolated and injected into the Debuncher, where the antiproton bunches are spread to form a continuous beam. This beam is then "cooled" (i.e. its average transverse momentum is reduced) via a process called stochastic cooling, where the trajectory of the beam is measured on one side of the Debuncher and corrected on the other. The antiproton beam is then injected into the Accumulator, where the beam is stored and stochastically cooled for several hours, until $\sim 10^{12}$ antiprotons have been collected. The antiprotons are collected into bunches and then injected backwards into the Main Injector at $8 \mathrm{GeV}$. In the Main Injector the antiprotons are accelerated to $150 \mathrm{GeV}$ and are then injected backwards into the Tevatron, where they are accelerated to a final energy of $980 \mathrm{GeV}$. The protons and antiprotons circulate within the Tevatron in opposite directions; they collide with a center of mass energy of $1.96 \mathrm{TeV}$ at the interaction points $\mathrm{B} \varnothing$ and $\mathrm{D} \varnothing$. 


\begin{tabular}{c|c}
\hline center of mass energy & $\sqrt{s}=1.96 \mathrm{TeV}$ \\
number of protons per bunch & $n_{p}=237 \times 10^{9}$ \\
$n_{\bar{p}}=22 \times 10^{9}$ \\
number of antiprotons per bunch \\
revolution frequency & $f=2.5 \mathrm{MHz}=(396 \mathrm{~ns})^{-1}$ \\
\hline number of proton bunches & 36 \\
number of antiproton bunches & 36 \\
interactions per bunch crossing & 2.3 \\
\hline
\end{tabular}

Table 3.1: Tevatron operational parameters from July 2001 through September 2003.

\subsubsection{Properties of $p \bar{p}$ collision at the Tevatron}

The number of events for a particular process at a given center of mass energy depends upon the cross section, e.g. Equation (2.5), and the instantaneous luminosity (i.e. the intensity of colliding proton and antiproton beams) integrated over the total data-taking period. The instantaneous luminosity is defined:

$$
\mathscr{L}=f \frac{n_{p} n_{\bar{p}}}{4 \pi \sigma_{p} \sigma_{\bar{p}}}
$$

where $f$ is the revolution frequency, $n_{p}$ and $n_{\bar{p}}$ are the average numbers of protons and antiprotons per bunch, and $\sigma_{p}$ and $\sigma_{\bar{p}}$ are the average cross-sectional areas of the bunches $\left(\sim 4 \mathrm{~mm}^{2}\right)$. The expected number of events for a particular process $i$ is then

$$
N_{i}=\sigma_{i} \times \int \mathscr{L} d t
$$

\subsection{The CDF detector}

The CDF detector is a general-purpose solenoidal detector with nearly complete coverage about the interaction region. The detector design emphasizes charged-particle tracking, lepton identification and fast projective calorimetry. The detector allows for the identification and characterization of electrons, muons, photons and jets in $p \bar{p}$ collisions at high energy. We describe the configuration of the CDF detector during run II. 


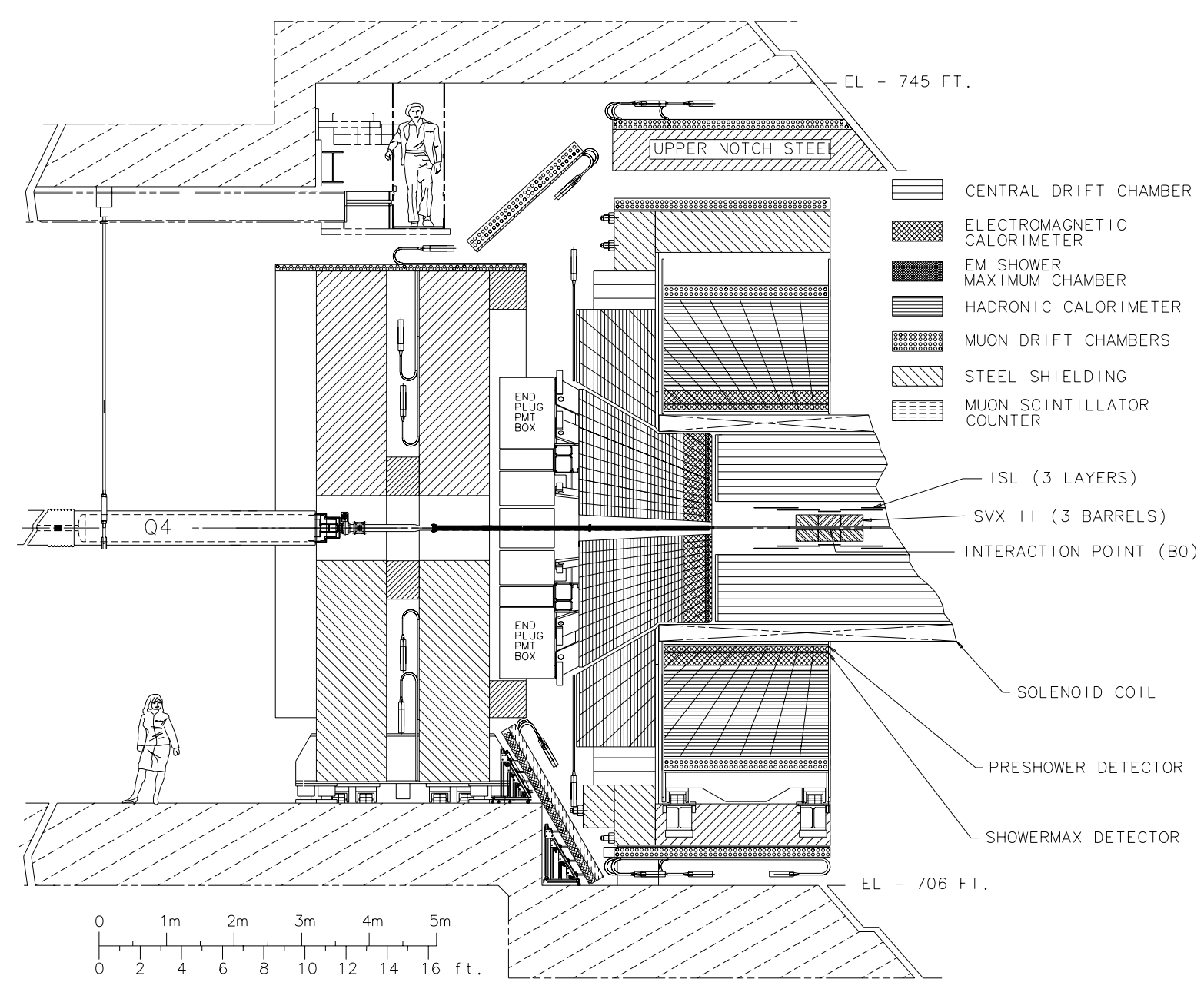

Figure 3.2: A cross-section view of the CDF detector.

The coordinate system used at CDF is right-handed: the $\hat{z}$ axis points along the direction of the proton beam, the $\hat{x}$ axis points out radially within the plane of the Tevatron. A cross-sectional view of the CDF detector is shown in Figure 3.2. The detector is symmetric about the $x-y$ plane.

Polar coordinates are commonly used: the polar angle $\theta$ is measured from the $\hat{z}$ axis; the azimuthal angle $\phi$ is measured from the $\hat{x}$ axis, in the $x-y$ plane. The coordinates 
$r, \phi$ and $\theta$ are defined

$$
\begin{aligned}
r & =\sqrt{x^{2}+y^{2}}, \\
\phi & =\tan ^{-1}\left(\frac{y}{x}\right), \\
\theta & =\tan ^{-1}\left(\frac{r}{z}\right) .
\end{aligned}
$$

The variable pseudorapidity is defined

$$
\eta=-\log \left(\tan \left(\frac{\theta}{2}\right)\right) .
$$

Pseudorapidity is a convenient variable to use: it is relatively insensitive to boosts along the $z$ axis, and the multiplicity of high-energy particles in $p \bar{p}$ collisions is roughly constant in $\eta$.

\subsubsection{Charged-particle tracking}

The central region of the CDF detector, $|\eta|<1.0$, is contained within a superconducting solenoid, $5 \mathrm{~m}$ in length and $3.2 \mathrm{~m}$ in diameter, which supports a $1.4 \mathrm{~T}$ magnetic field which is oriented parallel to the $z$ axis. Electrically charged particles produced within this volume will follow a helical trajectory; the particle's momentum in the $x-y$ plane and its electric charge are determined by observing the radius of curvature of the helix and its orientation relative to the magnetic field. The volume within the superconducting solenoid is instrumented by detectors which allow for precise determination of the trajectory of electrically charged particles. As charged particles pass through matter, they lose energy through the processes of ionization and bremsstrahlung. The tracking systems at CDF locate charged particles in space by sampling the deposited electrical charge due to ionization along the particle trajectory.

The Central Outer Tracker is an open-cell drift chamber with a large tracking volume, capable of measuring the momentum and position of charged particles with good preci- 


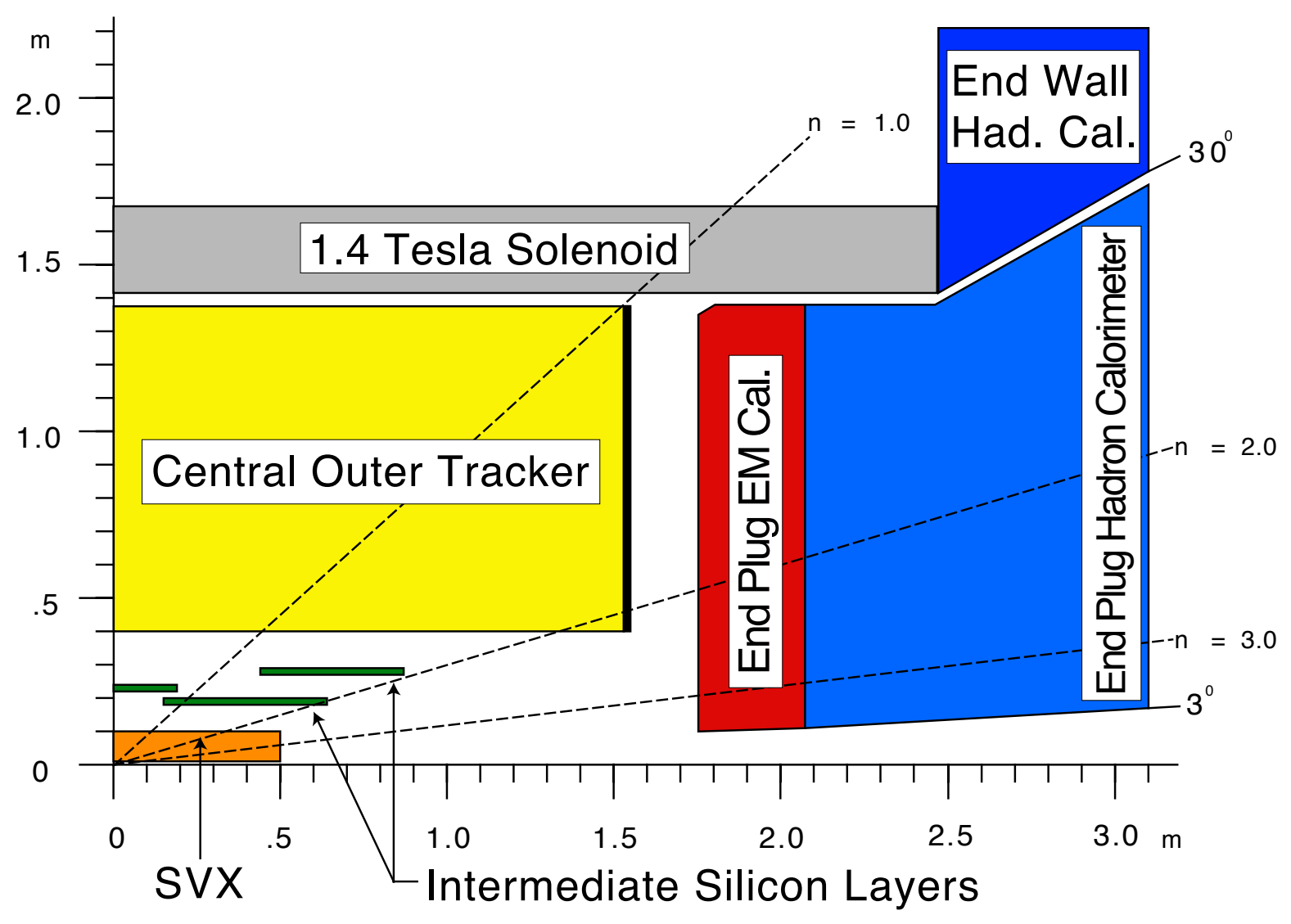

Figure 3.3: The CDF tracking volume.

sion [45]. The silicon microstrip detectors [46] (Layer 00, the Silicon Vertex Detector, and the Intermediate Silicon Layers) are capable of measuring the position of charged particles near the interaction region with excellent precision. A schematic view of the CDF tracking volume is shown in Figure 3.3.

\section{The silicon detectors}

The volume immediately surrounding the beam-pipe is instrumented by three silicon detector systems: Layer 00 (L00), the Silicon Vertex Detector (SVX), and the Intermediate Silicon Layers (ISL). A transverse view of the silicon detectors is shown in Figure 3.4.

The silicon detectors locate the charged-particle tracks with excellent spatial resolu- 


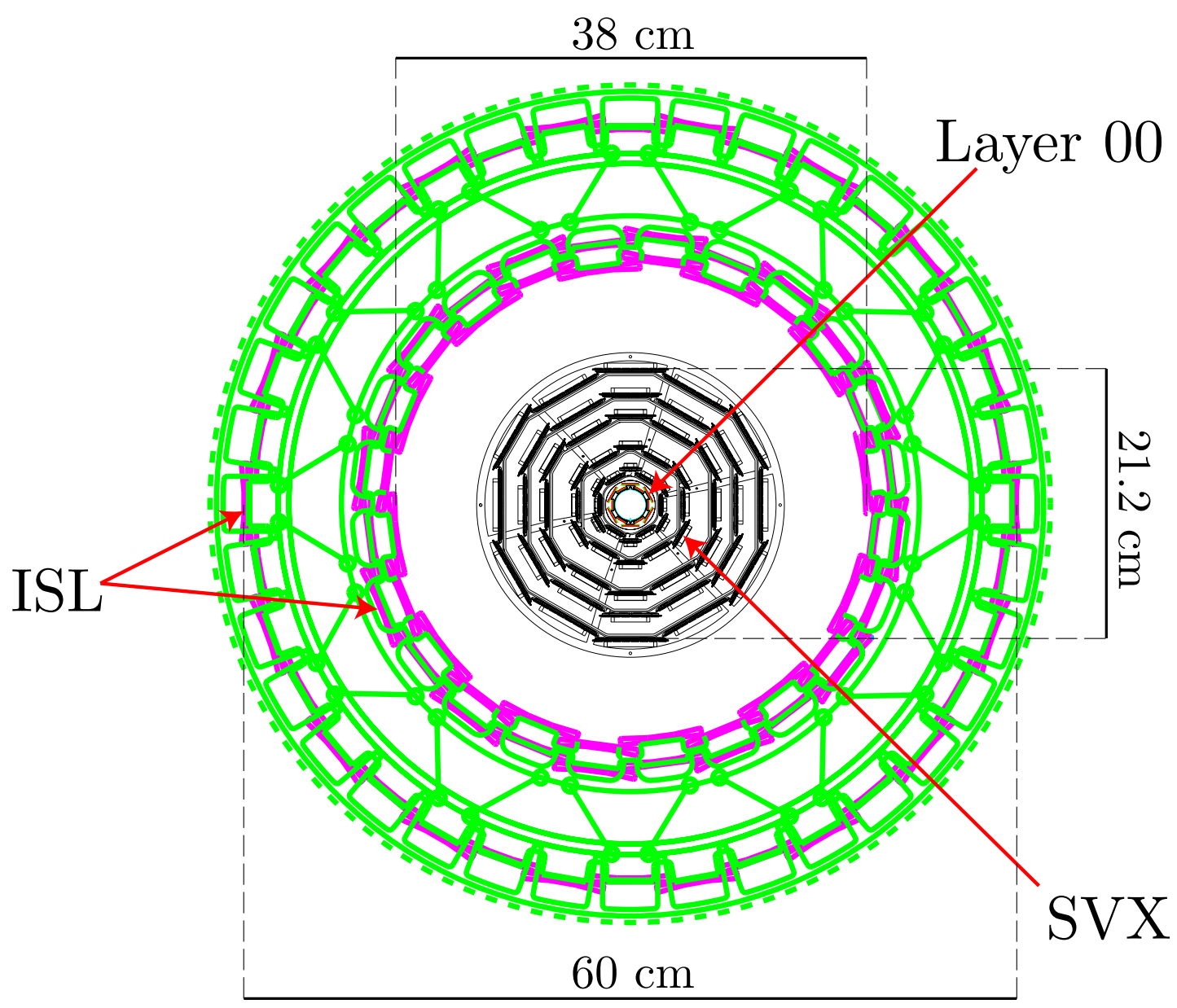

Figure 3.4: A transverse view of the CDF silicon detectors.

tion; tracks reconstructed with silicon information have an impact parameter resolution $\sigma_{d_{0}} \sim 60 \times 10^{-6} \mathrm{~m}$. Reconstructed jets which include tracks with data from the SVX can be identified as being consistent with the decay of a long-lived $b$ hadron, provided the reconstructed jet vertex is sufficiently displaced from the primary event vertex.

Layer 00 [47] is a single-sided radiation-hard silicon microstrip detector. It immediately surrounds the beryllium beam-pipe with an inner radius of $1.15 \mathrm{~cm}$ and an outer radius of $2.1 \mathrm{~cm}$. L00 is designed to enhance the track impact parameter resolution. At the time of this analysis, L00 was not fully commissioned and information from this system was not used. 


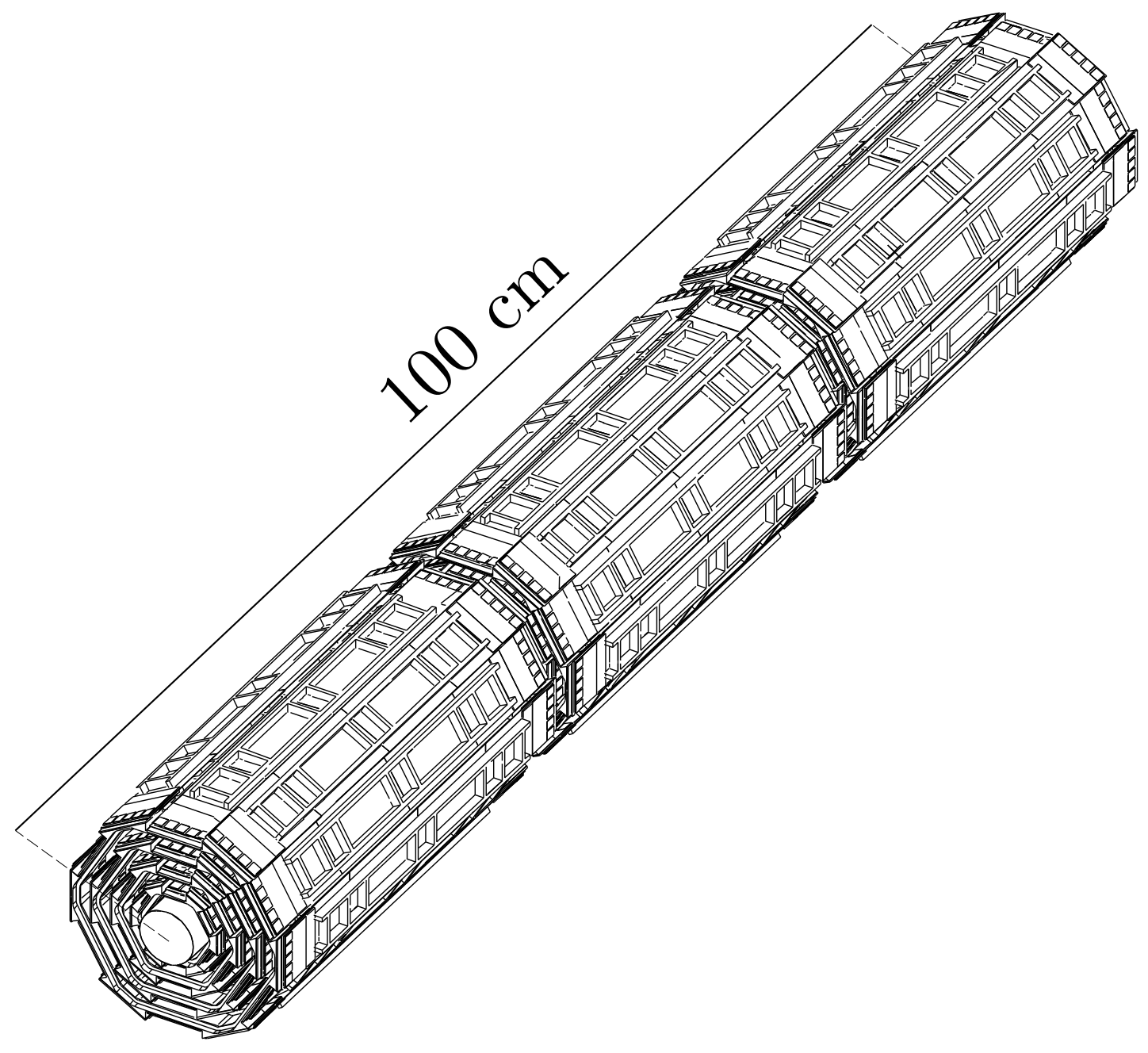

Figure 3.5: The SVX assembly.

The Silicon Vertex Detector (SVX) [46] is composed of five layers of double-sided silicon microstrip detectors; this detector covers the radial region from 2.5 to $10.6 \mathrm{~cm}$. The SVX detector is composed of six cylindrical barrels, each measuring $16 \mathrm{~cm}$ along the $z$ axis. The SVX detector possesses 12 -fold symmetry in $\phi$ (see Figure 3.4). The SVX can reconstruct three-dimensional tracks: one side of each silicon microstrip detector provides tracking information in the $r-\phi$ plane, the other side provides tracking information in the $r-z$ plane. Three of the five SVX layers provide $90^{\circ}$ stereo information, two SVX layers provide $\pm 1.2^{\circ}$ small-angle stereo information. The entire SVX assembly is illustrated in 


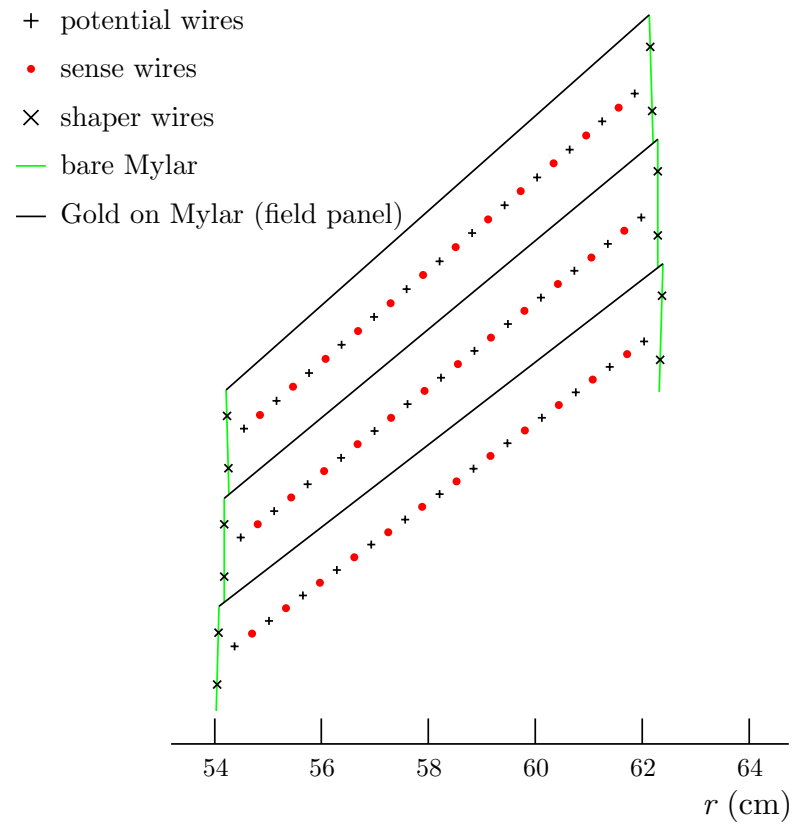

(a) The nominal cell layout for COT superlayer 2

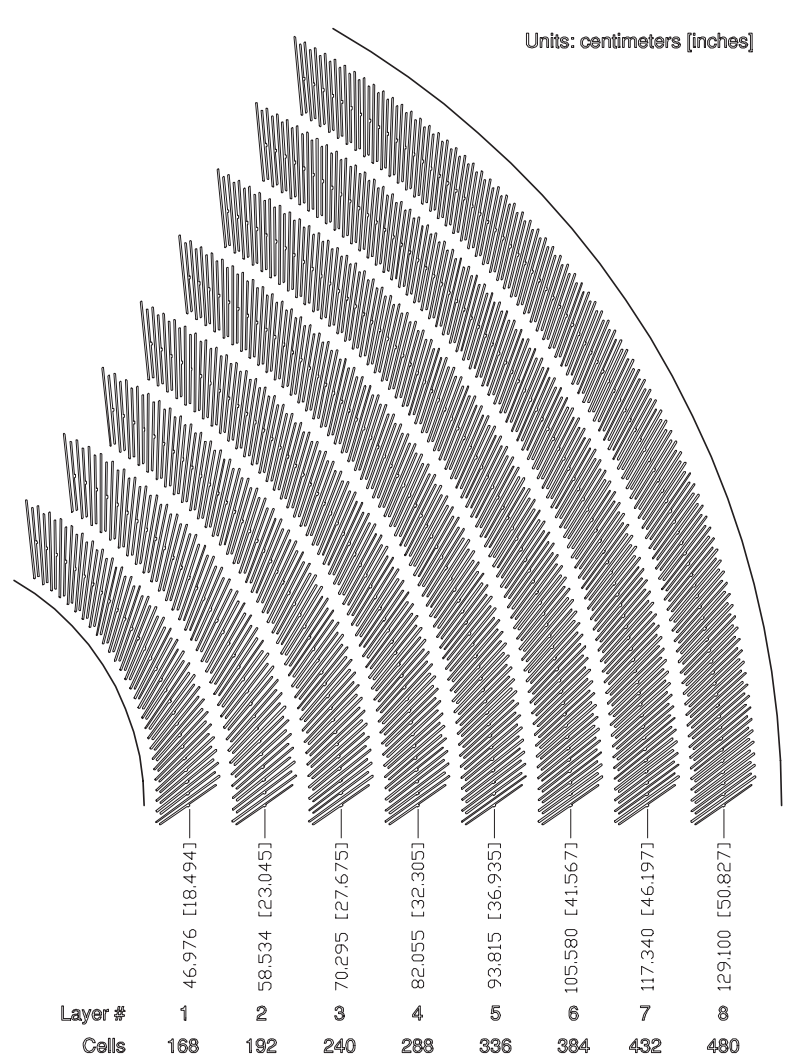

(b) $1 / 6^{\text {th }}$ of the COT east end plate

Figure 3.6: Transverse views of (a) the nominal cell layout for COT superlayer 2 , and (b) $1 / 6^{\text {th }}$ of the COT east end plate

Figure 3.5.

The Intermediate Silicon Layers (ISL) [48] are composed of overlapping layers of double-sided silicon microstrip detectors, staggered at radii between 19 and $30 \mathrm{~cm}$. The ISL improves the tracking coverage in the forward region of the detector. This system is useful for matching tracks within the Central Outer Tracker to those within the SVX.

\section{The central outer tracker}

The Central Outer Tracker (COT) [45] is a cylindrical open-cell drift chamber. It is designed to measure the three-dimensional trajectories of charged particles in the central region, $|\eta|<1$. The COT occupies the radial region 40 to $138 \mathrm{~cm}$, and measures $310 \mathrm{~cm}$ along the $z$ axis. 
The COT volume is filled with a mixture of argon, ethane and isopropyl alcohol gases, at a ratio of $500: 500: 173$. The basic element of the COT design is the cell, a structure which spans the length of the COT. Within each cell are high-voltage field panels, potential wires and shaper wires which serve to support a regular electrostatic field. Each cell has twelve sense wires interspersed with the potential wires. A transverse view of typical cells is shown in Figure 3.6.

As charged particles pass through the cell, the gas mixture is ionized. The electrostatic field guides the ions towards sense wires; as the ions approach the sense wires the electric field strength increases and more ions are produced in a cascade. The charge deposited on the sense wire is amplified and the timing and charge deposition information of the "hit" is digitized.

The cells of the COT are arranged into eight superlayers. Four superlayers have their wires arranged parallel to the $z$ axis allowing for track measurements in the $r-\phi$ plane. Four superlayers have their wires offset $3^{\circ}$ from the $z$ axis so that stereo information may be recorded, allowing for track measurements in the $r-z$ plane. Given a detailed understanding of the geometry of the COT, its material composition, the topology of the magnetic field within and the timing and charge deposition information from hits on the sense wires, three-dimensional tracks can be reconstructed. Tracks with only COT tracking information have a momentum resolution $\delta p_{T} / p_{T}^{2}=0.0030 / \mathrm{GeV} / \mathrm{c}$; tracks with information from the COT and the silicon detectors have a momentum resolution $\delta p_{T} / p_{T}^{2}=0.0012 / \mathrm{GeV} / \mathrm{c}$

\subsubsection{The calorimeter systems}

The calorimeter systems at CDF measure the energy of particles by sampling the electromagnetic and hadronic showers produced as the particles traverse and interact 


\begin{tabular}{|c|c|c|c|}
\hline System & $|\eta|$ Range & Thickness & Energy Resolution \\
\hline \hline CEM & $0.0-1.1$ & $19 X_{0}, 1 \lambda$ & $13.7 \% / \sqrt{E_{T}} \oplus 2 \%$ \\
PEM & $1.1-3.6$ & $21 X_{0}, 1 \lambda$ & $16 \% / \sqrt{E} \oplus 1 \%$ \\
\hline CHA & $0.0-0.9$ & $4.5 \lambda$ & $50 \% / \sqrt{E_{T}} \oplus 2 \%$ \\
WHA & $0.7-1.3$ & $4.5 \lambda$ & $75 \% / \sqrt{E} \oplus 4 \%$ \\
PHA & $1.2-3.6$ & $7 \lambda$ & $80 \% / \sqrt{E} \oplus 5 \%$ \\
\hline
\end{tabular}

Table 3.2: Properties of the CDF II calorimeter systems. The energy resolutions for the electromagnetic calorimeters are for electrons and photons; the resolutions for the hadronic calorimeters are for isolated pions.

with regions of dense material. The general calorimeter design is comprised of alternating layers of inert, dense material (i.e. lead or iron) and layers of active scintillator which sample the shower energy.

These systems cover $2 \pi$ in azimuth and the pseudorapidity region $|\eta|<3.6$. The calorimeters are segmented into projective towers: alternating layers of scintillator and inert material, stacked to form a "tower" oriented towards the interaction region. Wavelengthshifting fibers (WLS) are embedded within the layers of scintillator, and transmit the scintillation light via acrylic light guides to photomultiplier tubes (PMTs) located at the tops of the towers. Integrating the charge collected by the PMT gives a measure of the energy deposited in the calorimeter. A typical calorimeter wedge composed of several towers is shown in Figure 3.7.

The CDF calorimeter is roughly divided into two regions: the central calorimeter covers the region $|\eta|<1.1$; the forward calorimeter covers the region $1.1<|\eta|<3.6$. Table 3.2 gives the segmentation of the whole of the CDF calorimeter for different regions of pseudorapidity, as well as the energy resolution and thickness of these systems.

Each calorimeter system consists of two components: an electromagnetic component and a hadronic component. The electromagnetic calorimeter measures the energy of elec- 


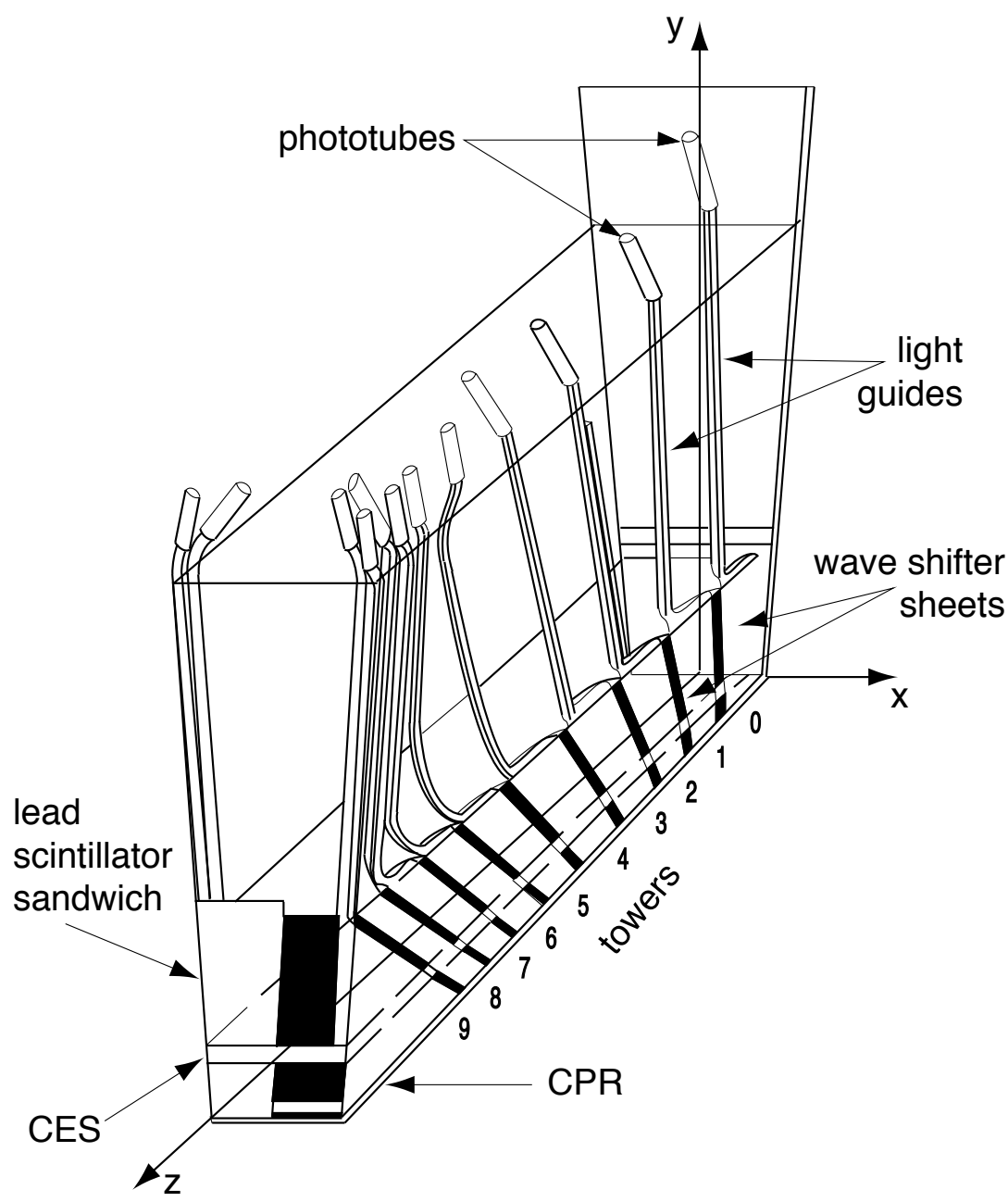

Figure 3.7: A typical central calorimeter wedge, showing the electromagnetic lead/scintillator stack, the wavelength-shifting sheets, and the strip chamber.

trons and photons by sampling the electromagnetic showers initiated by bremsstrahlung of the electron, or $e^{+} e^{-}$pair production of the photon. The hadronic calorimeter measures the energy of single hadrons and jets (due to the fragmentation of quarks or gluons) by sampling the electromagnetic showers due to neutral meson production and their subsequent electromagnetic decay, and the hadronic showers due to strong interactions of hadrons and mesons with the heavy atomic nuclei. 


\section{The central calorimeter}

The Central Electromagnetic Calorimeter (CEM) [49] towers consists of alternating layers of lead and polystyrene scintillator with a total thickness of 19 radiation lengths ${ }^{1}$. The CEM features two proportional chambers, used to characterize the evolution of the electromagnetic shower within the tower by observing its lateral profile. The shower maximum detector (CES) is a argon-carbon dioxide proportional chamber which sits six radiation lengths within the CEM, where electromagnetic showers reach their maximum lateral extent. The central pre-radiator $(\mathrm{CPR})$ is a proportional chamber which sits between the first lead layer and the solenoid. Together these wire chambers are used to improve efficiency of electron and photon identification as well as the the purity of these samples.

The central hadronic calorimeter (CHA) [50] consists of alternating layers of iron and scintillator, with a total thickness of 4.5 nuclear interaction lengths ${ }^{2}$. The design and function is similar to that of the CEM.

\section{The end-wall and plug calorimeters}

To compensate for the limited forward coverage of the CHA, the end-wall hadronic calorimeter (WHA) covers the region $0.7<|\eta|<1.3$. The plug calorimeter covers the region $1.1<|\eta|<3.6$. The EM component (PEM) consists of alternating layers of lead and scintillator with a total thickness of 21 radiation lengths. Pre-shower and shower max wire chambers are embedded within the PEM. The hadronic component of the plug calorimeter (PHA) has a thickness of seven nuclear interaction lengths.

\footnotetext{
${ }^{1}$ The radiation length, $X_{0}$, is defined as the distance over which a high-energy electron loses all but $1 / e$ of its energy by bremsstrahlung.

${ }^{2}$ The nuclear interaction length, $\lambda$, is defined as the mean free path of a particle before undergoing an inelastic nuclear interaction.
} 


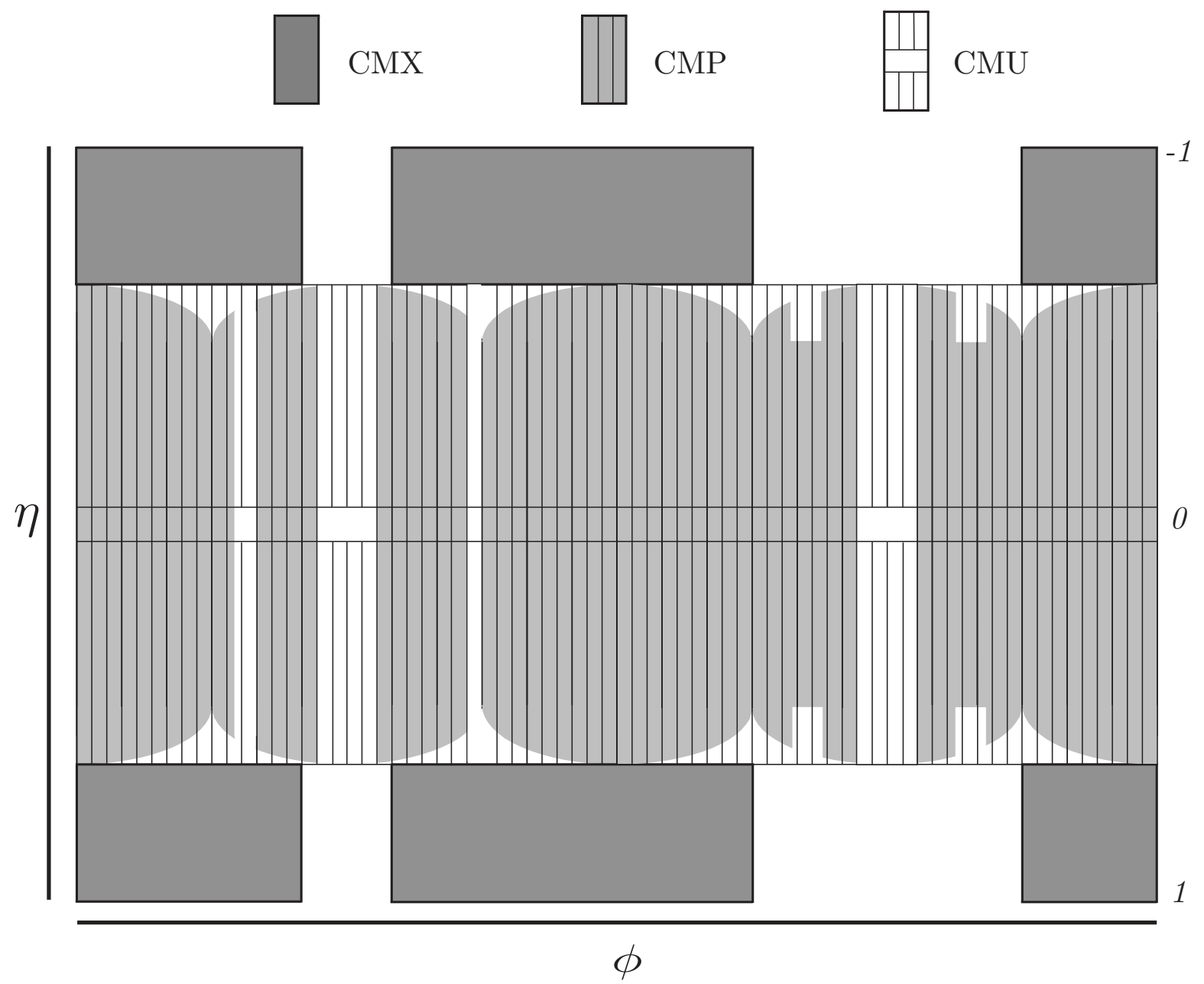

Figure 3.8: A map of the coverage of the CDF muon systems.

\subsubsection{The muon detectors}

Due to their relatively large mass, muons interact with matter through bremsstrahlung at a much lower rate than electrons. Muons lose energy primarily through ionization, though they rarely produce electromagnetic showers. Because energetic muons tend to traverse regions of dense material without great energy loss, the CDF muon detectors are generally the outermost detector systems. These systems are separated from the rest of the detector by steel shielding; this shielding serves to absorb charged pions which can traverse the whole of the hadronic calorimeter and could be interpreted as muons. 


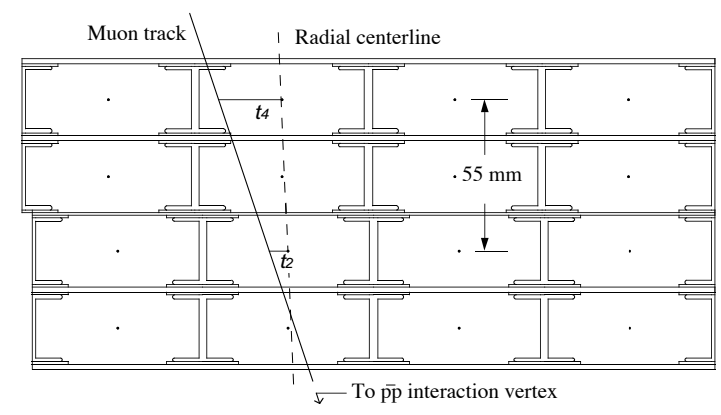

(a) A transverse view of a CMU module

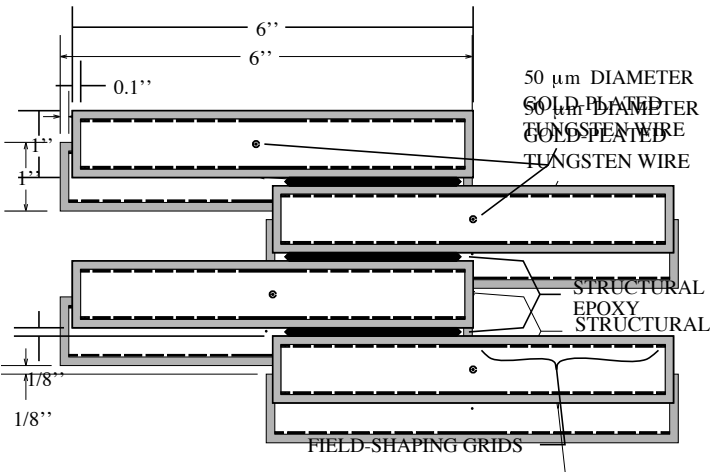

(b) A transverse view of a CMP stack

Figure 3.9: The central muon detector elements.

The CDF muon detectors [51] consist of stacked argon-ethane drift tubes, some backed with scintillation counters. A map of the geometrical coverage of the CDF muon systems is shown in Figure 3.8. Muons which pass through the drift tubes leave a trail of ionized gas along their trajectory; muons which pass through the scintillator panels induce scintillation light pulses which are collected by PMTs. A coarse measurement of the muon trajectory can be reconstructed given charge deposition and timing information from the drift tubes, and timing information from the scintillators.

\section{Central muon detectors}

The central muon detector (CMU) consists of rectangular cells, consisting of a sense wire within a regular electrostatic field. The CMU is composed of 144 modules, each with 16 cells, as illustrated in Figure 3.9(a).

The central muon upgrade (CMP) sits behind $60 \mathrm{~cm}$ of steel shielding. The CMP consists of four layers of staggered rectangular drift tubes. The outer surface of the CMP is covered by the central scintillator upgrade (CSP), a layer of scintillator tiles read out by PMTs. Figure 3.9(b) shows a typical CMP stack. The central muon extension (CMX) consists of conical sections of drift tubes, and covers the pseudorapidity range 
$0.65<|\eta|<1.0$. Each section of CMX has $15^{\circ}$ of azimuthal coverage and consists of 12 drift tubes stacked in four layers. The outer surface of the CMX is covered by the Central Scintillator Extension, (CSX) a layer of trapezoidal scintillator tiles, read out by PMTs.

\subsubsection{Trigger and data acquisition}

The overwhelming majority of proton-antiproton interactions at $\sqrt{s}=1.96 \mathrm{TeV}$ are due to phenomena which are not relevant to the study of the top quark. CDF employs an event trigger and a data acquisition system (DAQ) which are constructed to select events which are likely due to relevant physical phenomena and to reject uninteresting events. The event trigger is a three-level pipelined system. At each successive level a more detailed examination of the event is performed and the event accept rate is reduced. Collisions occur at a rate of approximately $2.5 \mathrm{MHz}$. To accommodate the limitations inherent to the process of physically recording event information for later detailed analysis, the trigger system outputs accepted events at a rate of approximately $100 \mathrm{~Hz}$.

\section{Level 1 trigger}

The Level 1 trigger (L1) uses custom hardware and coarse detector information to quickly select events. Within the DAQ electronics of each detector component, there is a 42 "bucket" data pipeline. The pipeline is synchronized with the Tevatron master clock, which has a period of 132 ns. For each proton-antiproton bunch crossing, event data enters the pipeline. A trigger decision must be made before the data reaches the end of the pipeline; if no decision is made, the data are lost. The decision time for the L1 trigger is $5.544 \mu \mathrm{s}$. During this decision time, data from the calorimeter, the COT, and the muon detectors are collected and fed into three synchronous streams. The calorimeter stream decision is based upon the energy deposited in calorimeter towers, along with the magnitude of unbalanced transverse energy. The eXtremely Fast Tracker (XFT) uses 
information from the COT to reconstruct tracks; events are (in part) accepted or rejected based on the multiplicity and transverse momenta of these tracks. The muon stream uses information from the XFT to match tracks to hits in the muon chambers to produce muon candidates. The data used in this analysis were collected while the Tevatron operated with a bunch spacing of $396 \mathrm{~ns}$. The maximum accept rate for the L1 trigger is $20 \mathrm{kHz}$; the nominal accept rate during the period of data taking for this analysis was $12 \mathrm{kHz}$.

\section{Level 2 trigger}

Events which meet the requirements of the L1 trigger are passed to the Level 2 (L2) trigger. At L2, an event is written into one of four buffers within the DAQ electronics for each detector component. These buffers differ from the data pipeline in that data are resident in the buffer until a decision is made, and will not be lost. While event data in a buffer are being processed, they cannot be overwritten by another event from L1. If a L1 accept does occur while all four L2 buffers are occupied, then "deadtime" is incurred. In order to minimize deadtime, the latency of the L2 decision must be less than approximately $80 \%$ of the average time between L1 accepts. Therefore, the L2 latency is designed to be $20 \mu$ s.

The L2 trigger is controlled by a DEC Alpha processor, which is programmed to identify primitive physics objects in event data. The L2 trigger uses all of the data available to the L1 trigger, as well as information from the CES and SVX detectors. The data available to the L2 trigger are more detailed than that which is available to the L1 trigger, allowing for better resolution, better purity and more stringent cuts. The L2 trigger is designed to work with a maximum accept rate of $300 \mathrm{~Hz}$. The accept rate during data taking varied from $100 \mathrm{~Hz}$ to $300 \mathrm{~Hz}$, depending on the instantaneous luminosity. 


\section{Level 3 trigger}

The Level 3 (L3) trigger consists of two components: the event builder and the processing farm. The event builder uses custom hardware to assemble all detector and trigger data from a single event. The processing farm is composed of 16 sub-farms; each sub-farm consists of 16 dual-CPU processing nodes. These, along with their networking infrastructure, constitute the L3 trigger.

Once an event is accepted at L2, the trigger data in the L2 buffers are collected and sent via optical fibers to the event builder. The event builder then collects all detector data which correspond to the same bunch crossing as the L2 accept. In this way, data from multiple bunch crossings are not mixed. Once all event data have been collected, they are fed to the L3 processing farm for complete event reconstruction. A trigger decision is then made based upon detailed particle identification and event topology. Once an event is accepted, it is sent from the processing node to the offline processing farm and eventual permanent storage. The accept rate from the L3 trigger was approximately $75 \mathrm{~Hz}$ during the data-taking period of this analysis.

\section{Data processing}

Events which pass a L3 trigger are collected and processed with the CDF offline reconstruction software. The reconstruction software applies detector calibration information to the raw data. From these data, physics objects such as electrons, muons, jets, etc. can be reconstructed. Once the raw data of an event have been reconstructed, the event is written to magnetic tape for permanent storage and future analysis. 


\section{CHAPTER 4}

\section{Data samples}

In Chapter 2 we discussed the most prolific channels for top-quark production and decay at the Tevatron. Here we describe the event selection used in this analysis to isolate top-enriched data in the lepton+jets and dilepton channels. We present estimates of the background content of these samples, and we describe for each sample the models of signal and background used in this analysis.

\subsection{The lepton+jets sample}

\subsubsection{Event selection}

The event selection for the lepton+jets sample is well-described elsewhere [52]. With this selection, we identify events consistent with the lepton+jets signature of $t \bar{t}$ decay. Here we summarize the selection.

\section{High $-P_{T}$ charged-lepton identification}

At Levels 1 and 2 of the CDF trigger, electrons are required to have an XFT track with $p_{T} \geq 8 \mathrm{GeV} / \mathrm{c}$ matched to an EM cluster with $E_{T} \geq 16 \mathrm{GeV}$. The ratio of hadronic to electromagnetic energy for this cluster must be less than 0.125. Muons are required to have an XFT track with $p_{T} \geq 8 \mathrm{GeV} / \mathrm{c}$ matched to muon stubs in the joint CMUP configuration or in the CMX. A complete version of the offline lepton selection is performed 
at Level 3, and repeated in offline processing with updated calibration constants.

\section{Offline electron selection}

Offline, electrons are required to have an EM cluster with $E_{T} \geq 20 \mathrm{GeV}$ matched to a track with $p_{T} \geq 10 \mathrm{GeV} / c$. The cluster is required to have an electromagnetic fraction and shower shape consistent with that of an electron. The extrapolated track is required to match the shower location as measured in the shower maximum strip detector, and to have a momentum consistent with the shower energy. Finally, since the electron from $W$ decay is expected to be isolated from other energy deposits in the calorimeter, the energy in a cone of radius $\Delta R=0.4$ around the electron cluster, but not including the cluster itself, is measured, and the ratio of the energy in the cone to the energy of the electron is required to be less than 0.1 .

Photon conversions in the detector material are a source of electron backgrounds. Electrons that are part of an identified conversion pair are rejected.

\section{Offline muon selection}

Offline, muons are required to have a COT track with $p_{T} \geq 20 \mathrm{GeV} / c$ matched to a CMUP or CMX muon stub. The matching is based on an extrapolation of the track to the outer muon chambers, where the effects of multiple scattering are taken into account. The energy in the calorimeter tower containing the muon is required to be consistent with the deposition expected for a minimum-ionizing particle. Backgrounds from cosmic rays are removed by requiring that the track originates at the origin of the detector coordinate system, and that the tower energy deposit is within a narrow timing window around the time of the beam crossing. 


\section{Missing transverse energy $\left(E_{T}\right)$}

Neutrinos from the process $W \rightarrow \ell \nu$ are not observed. However, we deduce their presence and their energy when we observe a large imbalance of energy deposited in the central calorimeter wedges. The $\mathbb{E}_{T}$ is calculated as the vector sum of the energy in each calorimeter tower multiplied by the azimuthal direction of the tower. If isolated high-momentum muons are found in the event, the $\mathbb{E}_{T}$ is corrected by subtracting the muon energy in the calorimeter, and adding the muon $p_{T}$ to the vector sum. We require $E_{T} \geq 20 \mathrm{GeV}$

\section{Hadronic jets}

The final state partons from $t \bar{t}$ decay (and background processes) tend to hadronize quickly, and are manifest as showers of charged and neutral particles. In the lepton+jets channel of $t \bar{t}$ decay, there are four final state partons which can be detected as jets. To improve acceptance we require three or more hadronic jets per event. Jets are defined within a cone of radius $\Delta R=0.4$; they are required to have $E_{T} \geq 15 \mathrm{GeV}$ and $|\eta| \leq 2.0$. We remove energy deposited in calorimeter towers traversed by a primary electron, and correct the tower $E_{T}$ for the location of the primary vertex $z$ coordinate in calculating the jet energy.

\section{SECVTX $b$-tags}

We improve background rejection by requiring that at least one jet per event have a displaced secondary vertex (called a SECVTX $b$-tag, or a $b$-tag), indicating it is consistent with the decay of a long-lived $b$-hadron. ${ }^{1}$ The primary vertex for the event (i.e. the point from which all prompt tracks originate) is determined by extrapolating well-measured tracks to a common point of origin. Secondary vertex tagging is done on a per-jet basis,

\footnotetext{
${ }^{1}$ The mean $b$-hadron lifetime is $\tau \sim 2$ ps [17]. Because of the long lifetime, $b$-hadrons traverse a relatively large distance $(\sim 1 \mathrm{~mm})$ before hadronizing to form a jet.
} 


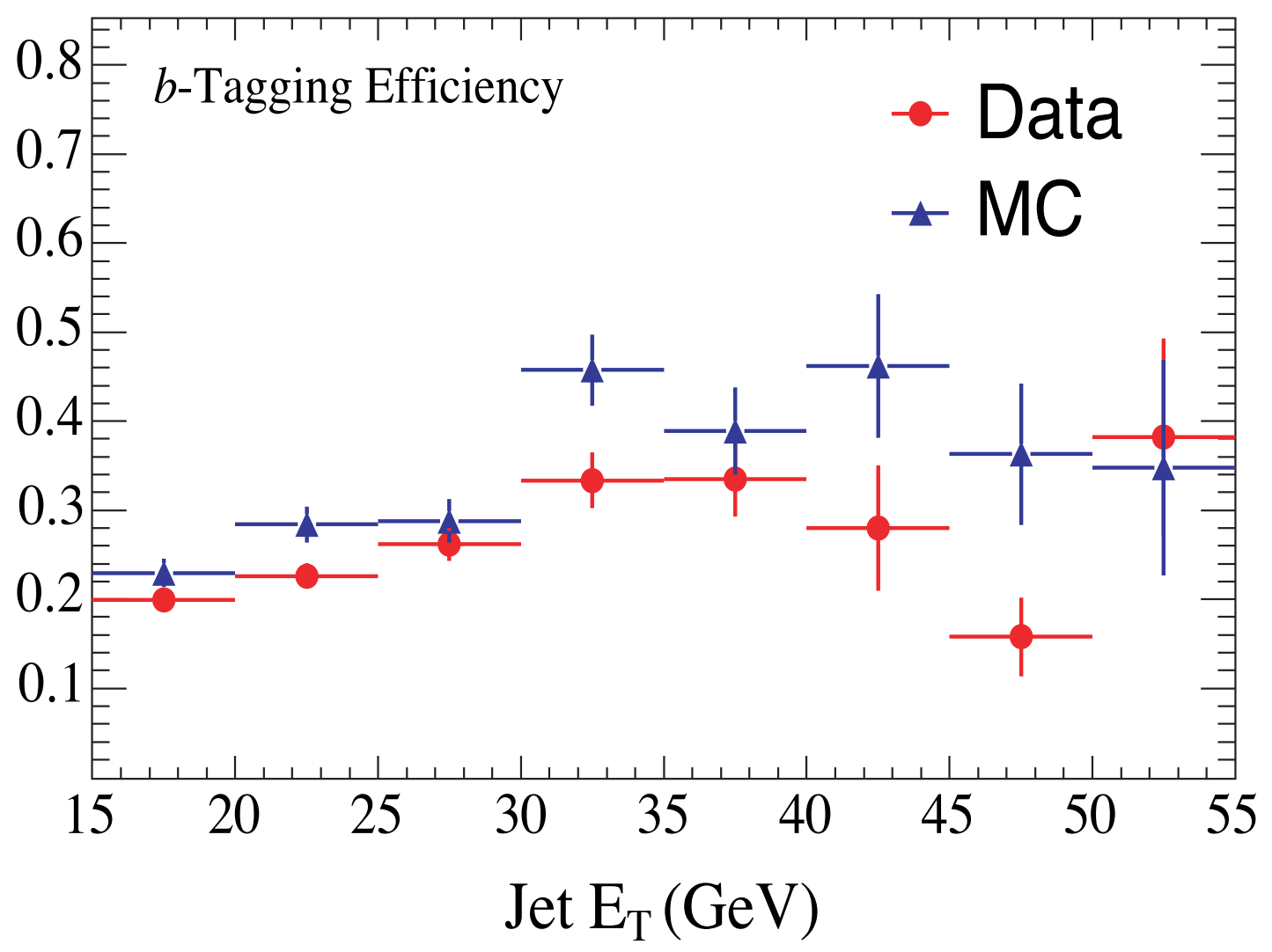

Figure 4.1: Efficiency to tag a $b$-jet as a function of jet $E_{T}$

where only tracks within the jet cone are considered. Cuts on the transverse momentum, the number of silicon hits attached to the tracks, the quality of those hits, and the $\chi^{2}$ of the final track fit are applied, and poorly reconstructed tracks are rejected. Only jets with at least two "good tracks" can produce a displaced vertex.

Displaced tracks in the jet are selected based on the significance of their impact parameter with respect to the primary vertex. Once a secondary vertex is found in a jet, the two-dimensional decay length of the secondary vertex $L_{2 D}$ is calculated as the projection onto the jet axis, in the $r-\phi$ plane, of the vector pointing from the primary vertex to the secondary vertex. Secondary vertices corresponding to the decay of $b$ - and $c$-hadrons are expected to have large positive $L_{2 D}$, while the distribution of secondary vertices from random poorly-measured tracks is expected to be symmetric about the primary vertex. 


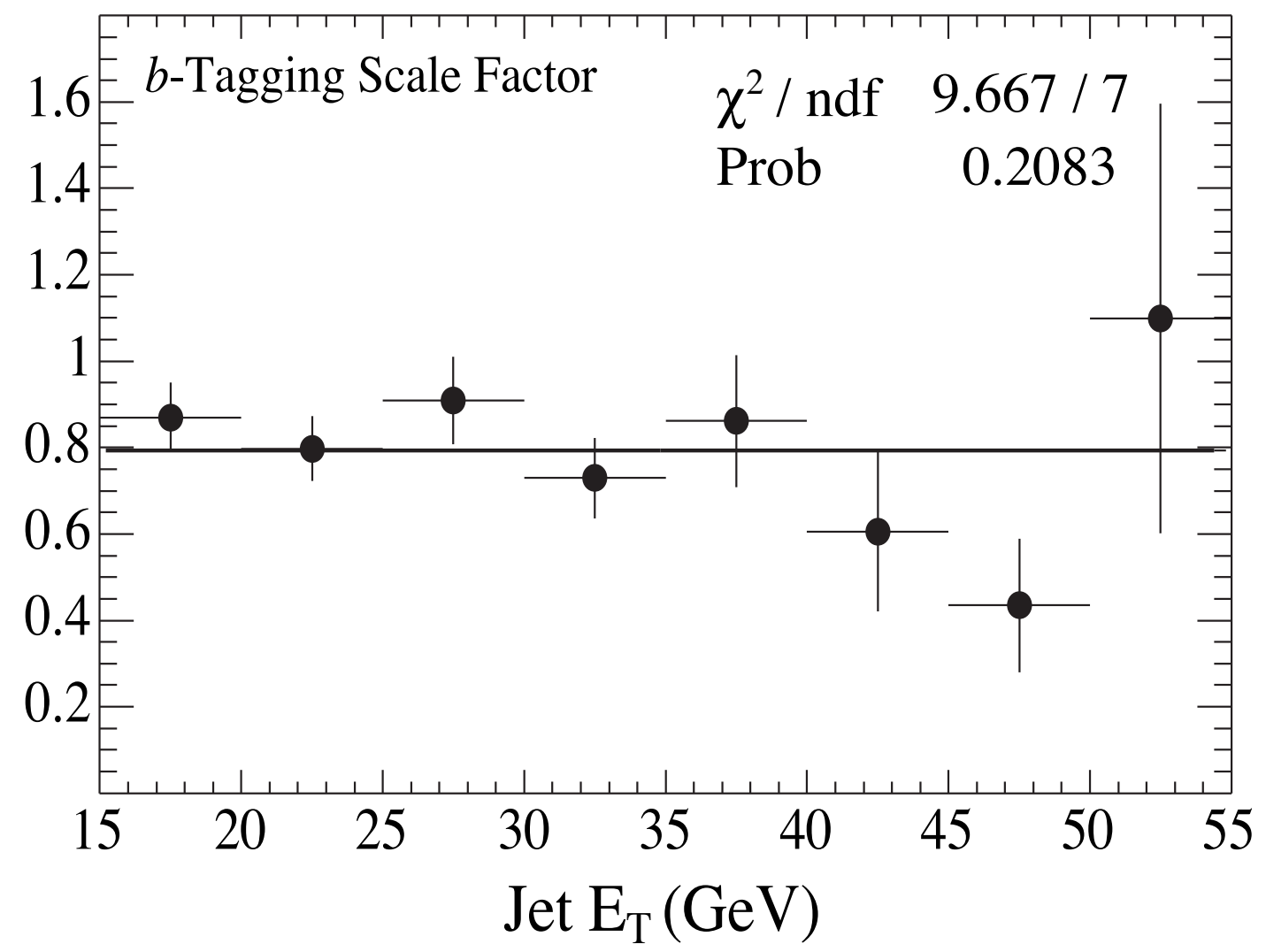

Figure 4.2: Data/Monte Carlo scale factor for tagging a $b$-jet, as a function of jet $E_{T}$.

To reduce the background from these false secondary vertices (mistags), secondary vertices are required to have $L_{2 D} / \sigma_{L_{2 D}}>3$, where $\sigma_{L_{2 D}}$ is the total estimated uncertainty on $L_{2 D}$, including the error on the primary vertex. A $b$-tagged jet is defined as be a jet containing such a secondary vertex.

The efficiency to tag a $b$-jet is estimated using a control sample in the data and a matching Monte Carlo sample. The tagging scale factor is defined as the ratio of efficiencies in data and Monte Carlo. This scale factor is used to correct the tagging efficiency in $t \bar{t}$ Monte Carlo samples, so that the geometrical acceptance and energy dependence of the tagger are taken from the simulation, but with the overall normalization determined from the data. Figure 4.1 shows the efficiency to tag a $b$-jet as a function of the jet $E_{T}$ in data and Monte Carlo. Figure 4.2 shows the $b$-tagging scale factor as a 
function of jet $E_{T}$; from this plot it is apparent that the data/Monte Carlo scale factor does not vary with jet $E_{T}$.

Vetos for $t \bar{t} \rightarrow$ dileptons and $Z \rightarrow \ell^{+} \ell^{-}$

The processes $Z \rightarrow \ell^{+} \ell^{-}$and $t \bar{t} \rightarrow$ dileptons contribute to the inclusive high $p_{T}$ lepton selection. To reduce this contribution, any event containing two objects which satisfy the lepton identification is removed. In addition, we remove those events where the second lepton is an electron in the plug calorimeter or a muon that fails the CMUP requirement, but has one CMU or CMP muon segment. Remaining $Z$ bosons are removed by requiring that there be no second object which forms an invariant mass between 76 and $106 \mathrm{GeV} / c^{2}$ with the primary lepton. For primary muons the other objects considered are oppositelycharged isolated tracks with $p_{T}>10 \mathrm{GeV} / \mathrm{c}$. For primary electrons the second objects considered are tracks, electromagnetic clusters, and jets with $E_{T}>15 \mathrm{GeV}$ and $|\eta| \leq 2.0$ which have fewer than three tracks and an electromagnetic energy fraction greater than $95 \%$.

Total transverse energy $\left(H_{T}\right)$

Events from $t \bar{t}$ production have, on average, a significantly greater total transverse energy $H_{T}$ than background events. The total transverse energy, i.e. the scalar sum of the transverse energy of all the kinematic objects in the event, including all jets with $E_{T}>8 \mathrm{GeV}$ and $|\eta|<2.5$, is required to be greater than $200 \mathrm{GeV}$.

\subsubsection{Estimates of background content}

We use the estimates of the background content of the tagged lepton+jets data presented in Reference [52]. To improve our sensitivity, we partition the data by primary lepton type, jet multiplicity, and SECVTX tag multiplicity. We consider eight individual 


\begin{tabular}{|c|c|c|}
\hline primary lepton type & CEM & CMUP+CMX \\
\hline \hline single-tag 3 jet events & 17 & 9 \\
single-tag 4 jet events & 13 & 10 \\
\hline double-tag 3 jet events & 2 & 1 \\
double-tag 4 jet events & 3 & 2 \\
\hline
\end{tabular}

Table 4.1: Yield of events in the $161 \mathrm{pb}^{-1}$ lepton+jets sample.

subsamples; the expected background content of each subsample is indicated in Tables 4.2 through 4.5 .

In Figure 4.3 we show distribution of charged-lepton $p_{T}$ from the $\geq 3$ jet lepton+jets sample, where one $b$-tag is required. The total integrated luminosity is $161.6 \mathrm{pb}^{-1}$ for the samples where the primary lepton is a CEM electron or a CMUP muon, and $149.8 \mathrm{pb}^{-1}$ for the sample where the primary lepton is a CMX muon. These data are plotted against the total signal and background expectation. The background normalizations are taken from tables 4.2 through 4.5. The signal component is normalized to correspond to the $t \bar{t}$-production cross section $\sigma_{t \bar{t}}=6.7_{-0.9}^{+0.7} \mathrm{pb}[30]$.

\subsubsection{Lepton+jets background models}

Tables 4.2 through 4.5 show the background normalization estimates for the lepton+jets separated by primary lepton type, jet multiplicity and SECVTX tag multiplicity. Using these estimates we assemble PDFs to model the overall background shape as a function of charged-lepton $p_{T}$.

For each lepton+jets sample we compose a model of the background probability density as a function of charged-lepton $p_{T}$ by assembling histograms representing the contribution of each physics process to the overall acceptance. These histograms are weighted according to the background estimates given in Tables 4.2 through 4.5. We model all but the QCD component of the background using events generated with Monte Carlo. We model the QCD background using real lepton+jets events where the primary lepton is non-isolated. 


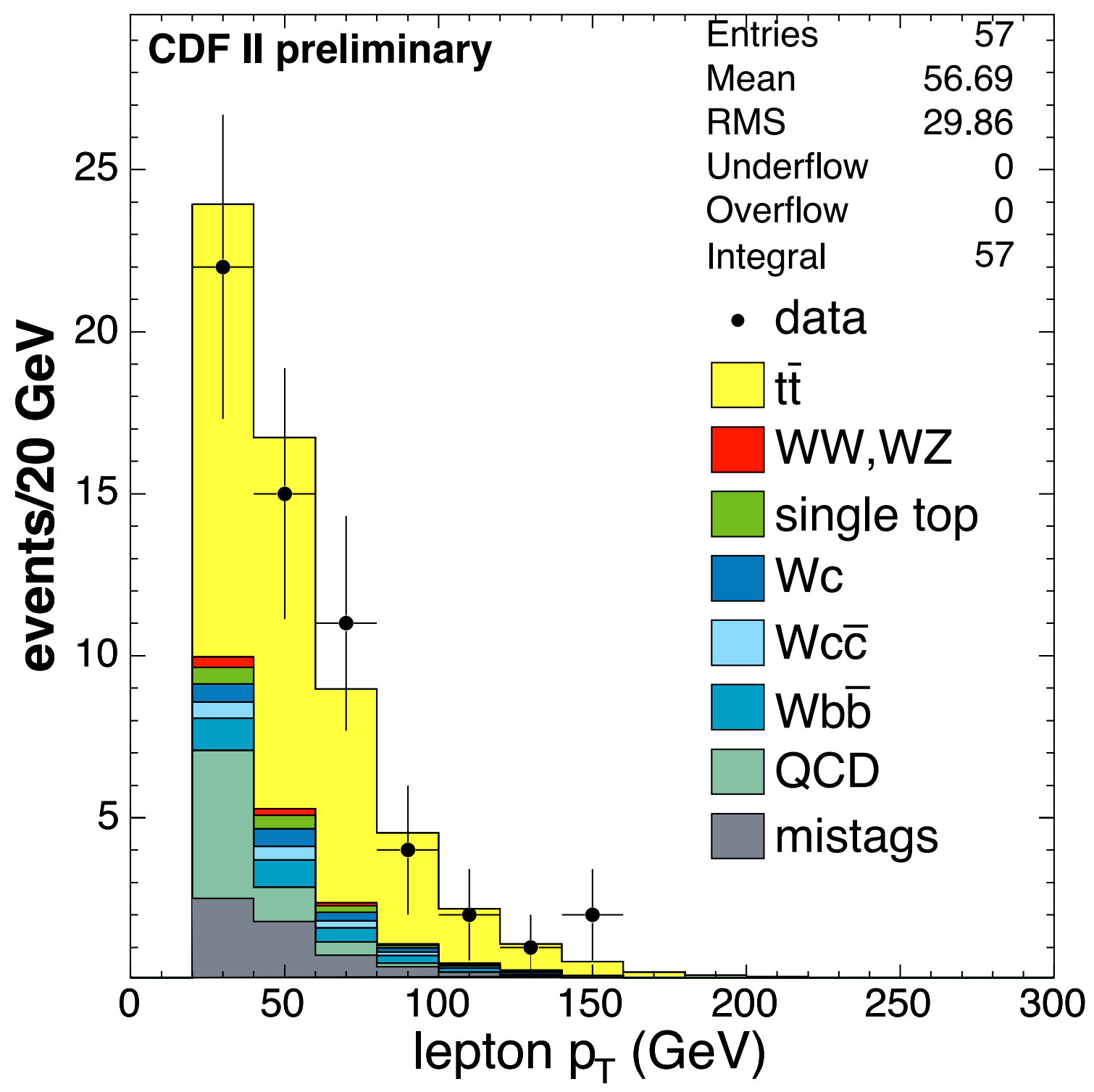

Figure 4.3: Distribution of charged-lepton $p_{T}$ in the $\geq 3$ jet SECVTX-tagged lepton+jets sample. 


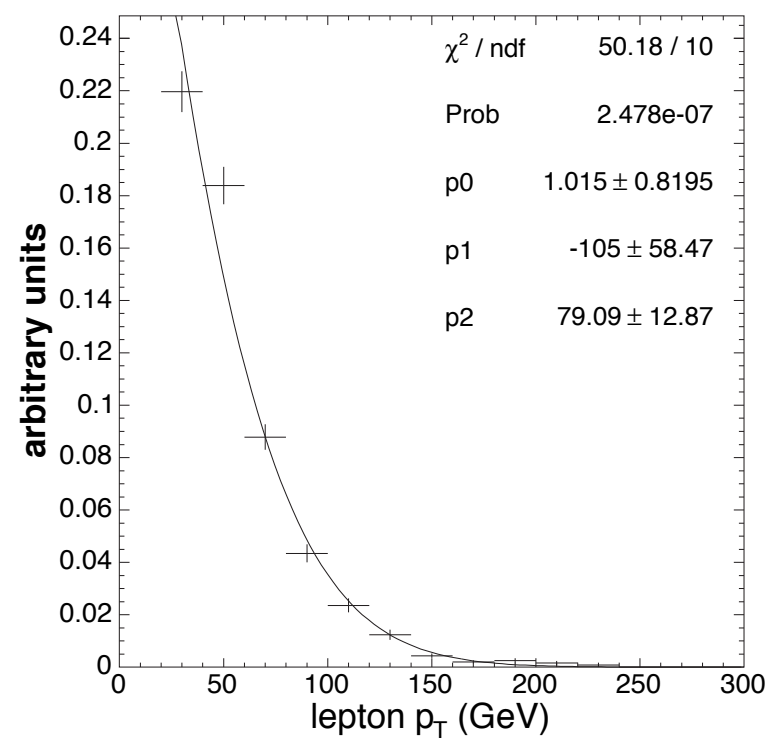

(a)

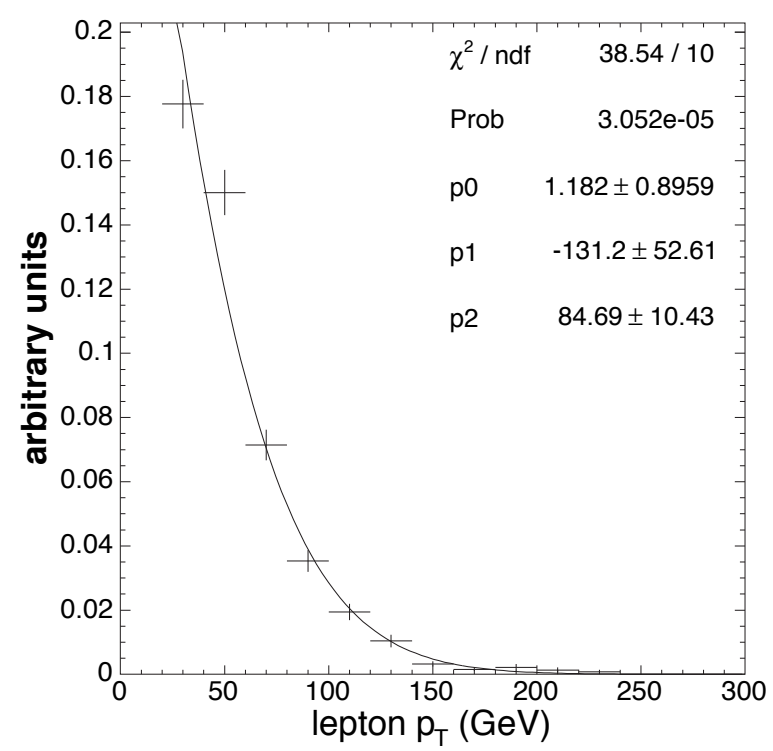

(c)

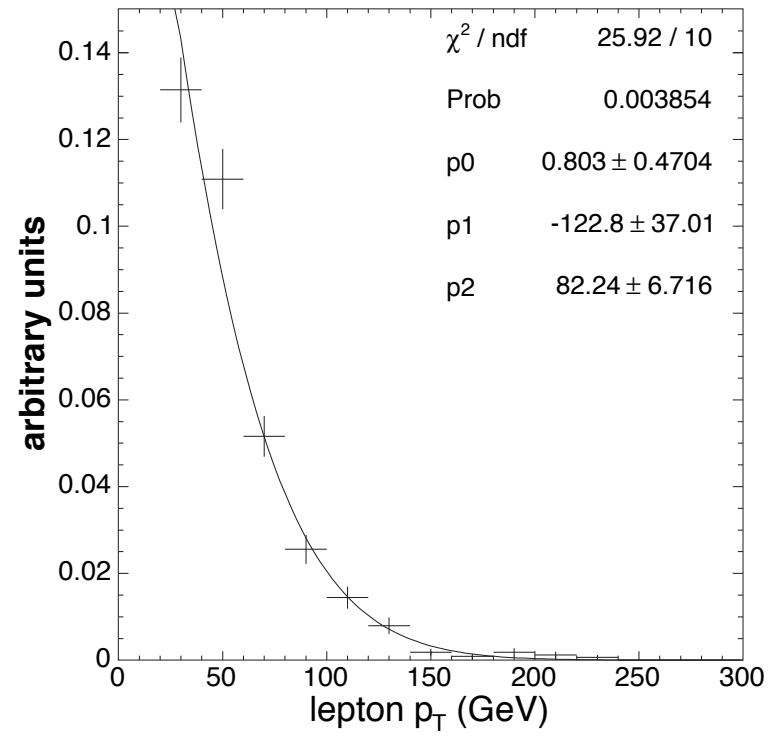

(b)

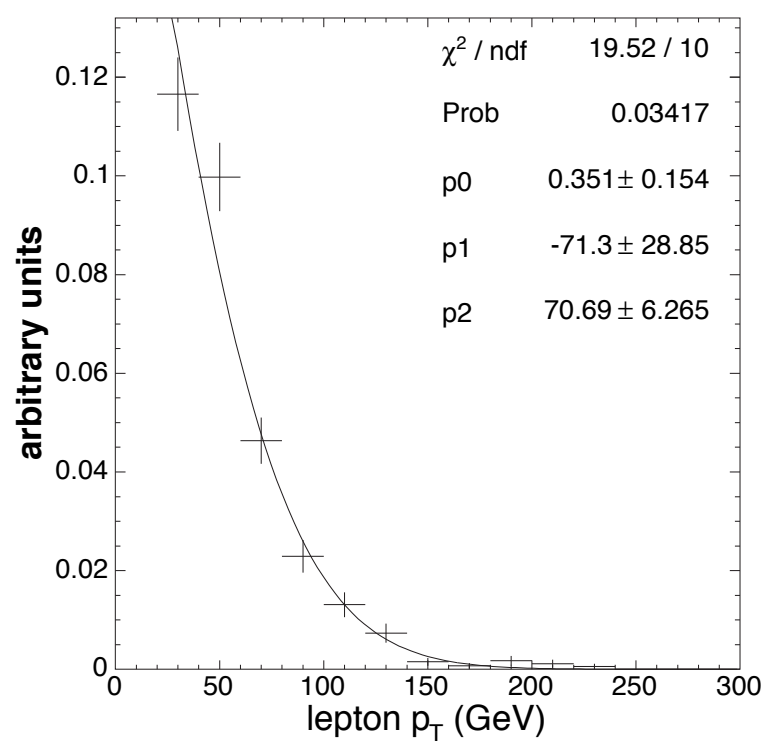

(d)

Figure 4.4: Parametrizations of the total expected backgrounds in the double-tag lepton+jet samples. (a) CEM 3-jet double-tag sample (b) CEM 4-jet double-tag sample (c) CMUP/CMX 3-jet double-tag sample (d) CMUP/CMX 4-jet double-tag sample. The functional form is that of a Gaussian function, $f(x) \propto \exp \left[\left(x-p_{1}\right)^{2} / 2 p_{2}^{2}\right]$. 


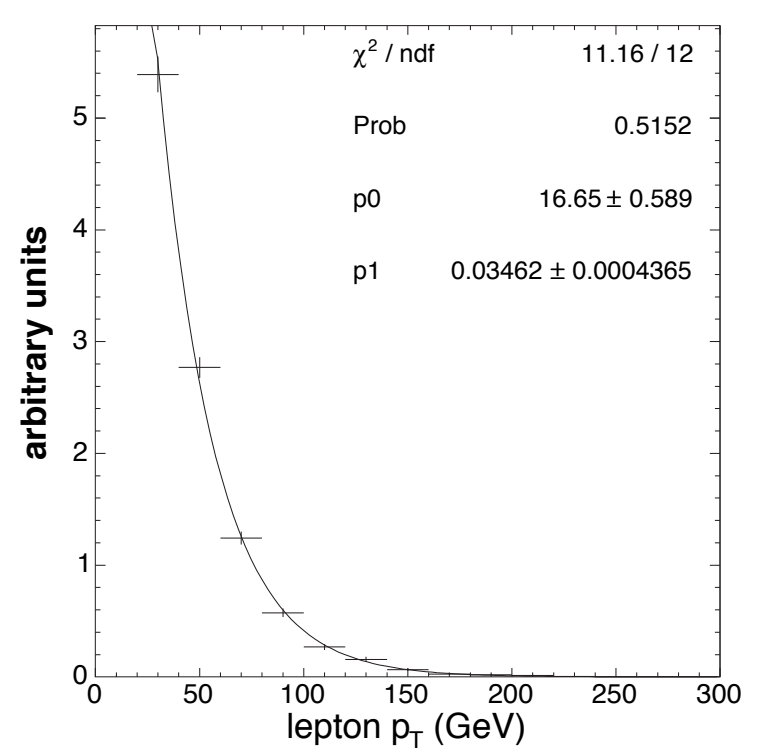

(a)

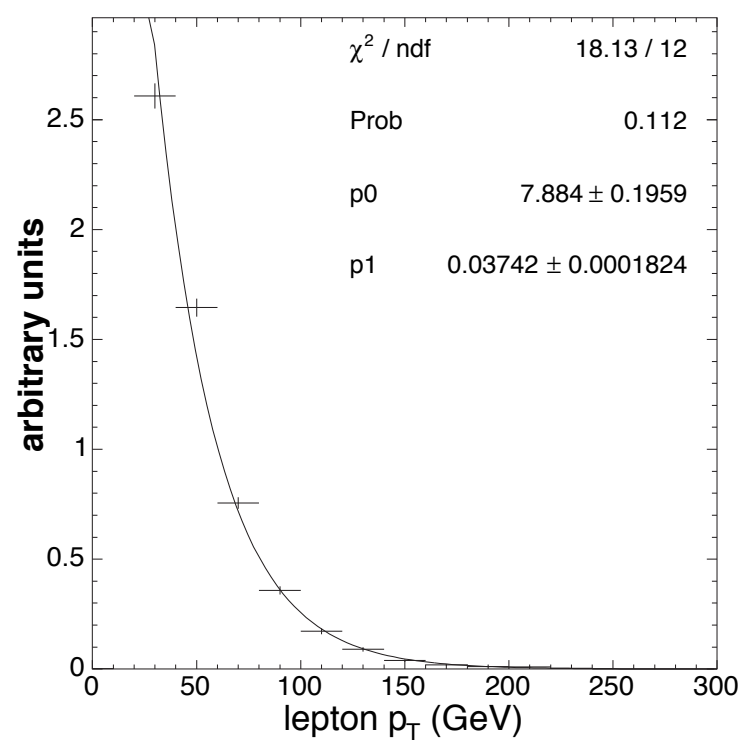

(c)

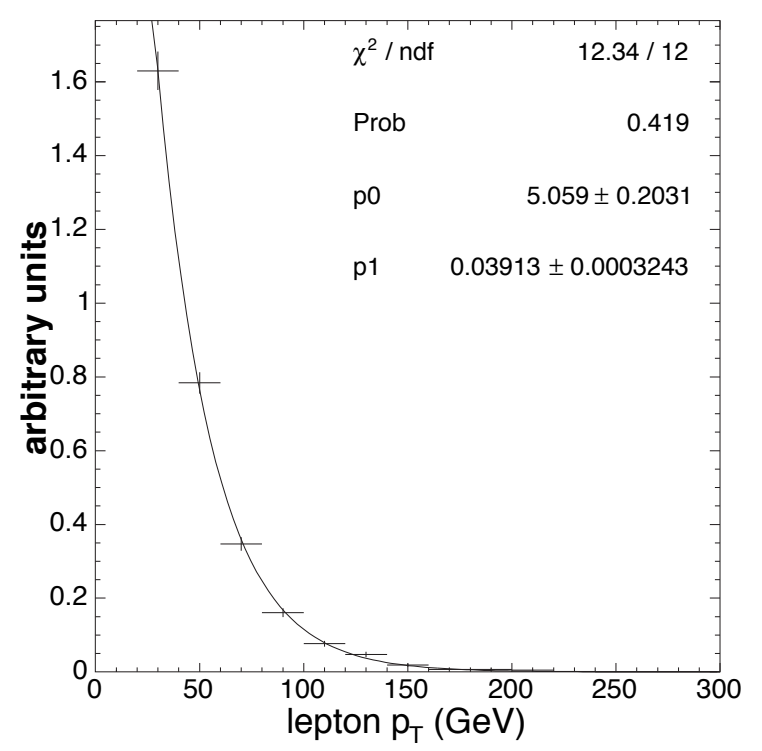

(b)

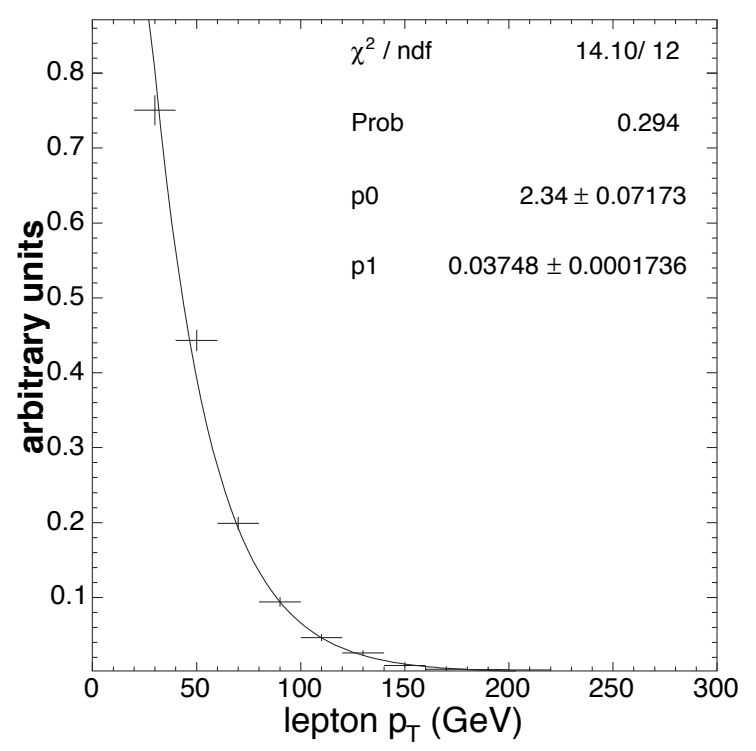

(d)

Figure 4.5: Parametrizations of the total expected backgrounds in the single-tag lepton+jets samples. (a) CEM 3-jet single-tag sample (b) CEM 4-jet single-tag sample (c) CMUP/CMX 3-jet single-tag sample (d) CMUP/CMX 4-jet single-tag sample. The functional form is $f(x) \propto \exp \left[-p_{1} x\right]$. 


\begin{tabular}{|c|c|c|}
\hline Background & $W+3$ jets & $W+\geq 4$ jets \\
\hline \hline Pretag & 241 & 69 \\
\hline QCD & $3.9 \pm 1.5$ & $1.3 \pm 0.5$ \\
single top & $0.6 \pm 0.1$ & $0.1 \pm 0.0$ \\
$W W / W Z$ & $0.3 \pm 0.1$ & $0.1 \pm 0.0$ \\
\hline mistags & $2.9 \pm 0.7$ & $0.8 \pm 0.3$ \\
$W b \bar{b}$ & $1.7 \pm 0.6$ & $0.4 \pm 0.2$ \\
$W c \bar{c}$ & $0.7 \pm 0.3$ & $0.2 \pm 0.1$ \\
$W c$ & $0.9 \pm 0.3$ & $0.1 \pm 0.1$ \\
\hline total background & $11.1 \pm 2.0$ & $2.9 \pm 0.6$ \\
\hline \hline observed & 17 & 13 \\
\hline
\end{tabular}

Table 4.2: Sample composition estimate of the 1 SECVTX-tag CEM+jets sample. All uncertainties include statistical and systematic errors.

\begin{tabular}{|c|c|c|}
\hline Background & $W+3$ jets & $W+\geq 4$ jets \\
\hline \hline single top & $0.08 \pm 0.04$ & $0.02 \pm 0.01$ \\
$W Z / W Z$ & $0.01 \pm 0.01$ & $0.01 \pm 0.01$ \\
\hline$W b \bar{b}$ & $0.23 \pm 0.08$ & $0.04 \pm 0.02$ \\
$W c \bar{c}$ & $0.02 \pm 0.01$ & $0.01 \pm 0.00$ \\
mistags & $0.04 \pm 0.01$ & $0.01 \pm 0.00$ \\
\hline total background & $0.39 \pm 0.10$ & $0.08 \pm 0.03$ \\
\hline \hline observed & 2 & 3 \\
\hline
\end{tabular}

Table 4.3: Sample composition estimate of the $\geq 2$ SECVTX-tag CEM+jets sample. All uncertainties include statistical and systematic errors.

We fit the resulting distribution to a simple form, either an exponential in the case of the single-tag samples, or a Gaussian in the case of the double-tag samples. The fits to the double-tag samples are shown in Figure 4.4. The fits to the single-tag samples are shown in Figure 4.5.

\subsubsection{Lepton+jets acceptance ratios}

We estimate the ratios of acceptances which parametrize the acceptance correction function discussed in section 5.1.1. These ratios are taken from the HERWIG Monte Carlo samples where the helicity of one of the $W \mathrm{~s}$ in $t \bar{t}$ events is fixed to be left-handed, longitudinal, or right-handed. The other $W$ in each event has left-handed polarization $30 \%$ of the time, and longitudinal polarization $70 \%$ of the time. We apply the event selection described in Section 4.1 with the exception that we do not require a SECVTX 


\begin{tabular}{|c|c|c|}
\hline Background & $W+3$ jets & $W+\geq 4$ jets \\
\hline \hline Pretag & 146 & 37 \\
\hline QCD & $1.1 \pm 0.6$ & $0.4 \pm 0.2$ \\
single top & $0.5 \pm 0.1$ & $0.1 \pm 0.0$ \\
$W W / W Z$ & $0.3 \pm 0.1$ & $0.0 \pm 0.0$ \\
\hline mistags & $1.7 \pm 0.3$ & $0.5 \pm 0.1$ \\
$W b \bar{b}$ & $1.2 \pm 0.4$ & $0.2 \pm 0.1$ \\
$W c \bar{c}$ & $0.5 \pm 0.2$ & $0.1 \pm 0.0$ \\
$W \bar{c}$ & $0.6 \pm 0.2$ & $0.1 \pm 0.0$ \\
\hline total background & $5.7 \pm 1.0$ & $1.4 \pm 0.3$ \\
\hline \hline observed & 9 & 10 \\
\hline
\end{tabular}

Table 4.4: Estimated background content of the SECVTX-tag CMUP/CMX+jets sample. All uncertainties include statistical and systematic errors.

\begin{tabular}{|c|c|c|}
\hline Background & $W+3$ jets & $W+\geq 4$ jets \\
\hline \hline single top & $0.06 \pm 0.04$ & $0.02 \pm 0.01$ \\
$W Z$ & $0.01 \pm 0.01$ & $0.00 \pm 0.01$ \\
\hline$W b \bar{b}$ & $0.16 \pm 0.05$ & $0.03 \pm 0.01$ \\
$W c \bar{c}$ & $0.01 \pm 0.00$ & $0.00 \pm 0.00$ \\
mistags & $0.01 \pm 0.00$ & $0.00 \pm 0.00$ \\
\hline total background & $0.25 \pm 0.07$ & $0.05 \pm 0.02$ \\
\hline \hline observed & 1 & 2 \\
\hline
\end{tabular}

Table 4.5: Estimated background content of the $\geq 2$ SECVTX-tag CMUP/CMX+jets sample. All uncertainties include statistical and systematic errors.

tag. The acceptance ratios are given in tables 4.8 and 4.9 .

\subsubsection{Lepton + jets signal models}

We construct probability density functions of charged-lepton $p_{T}$ for $t \bar{t}$ production and decay in the lepton+jets channel by applying the event selection described in Section 4.1.1 to $t \bar{t}$ events generated with the HERWIG program $[39,40]$, where the leptonicallydecaying $W$ has helicity $\lambda_{W}=-1,0$ or +1 (c.f. Section 2.3). After the selection we parametrize the probability density by fitting the resulting distributions to the function

$$
f(x)=L\left(x, p_{1}, p_{2}\right) \cdot G\left(x, p_{3}, p_{4}\right),
$$

where the second term is the familiar normal distribution

$$
G\left(x, p_{3}, p_{4}\right)=\frac{1}{p_{3} \sqrt{2 \pi}} \exp \left[-\frac{\left(x-p_{4}\right)^{2}}{2 p_{3}^{2}}\right],
$$




\begin{tabular}{|c|c|c|c|}
\hline \multicolumn{2}{|c|}{ jet multiplicity } & 3 & 4 \\
\hline \hline \multirow{3}{*}{ CEM } & $p_{1}$ & $38.4 \pm 1.0$ & $38.7 \pm 1.7$ \\
& $p_{2}$ & $11.1 \pm 1.0$ & $11.7 \pm 1.7$ \\
& $p_{3}$ & $76.3 \pm 2.9$ & $62.9 \pm 2.6$ \\
\hline \multirow{3}{*}{ CMUP/CMX } & $p_{1}$ & $38.6 \pm 1.2$ & $38.3 \pm 3.3$ \\
& $p_{2}$ & $11.3 \pm 1.1$ & $13.9 \pm 2.8$ \\
& $p_{3}$ & $72.8 \pm 2.9$ & $55.8 \pm 2.6$ \\
\hline
\end{tabular}

Table 4.6: Parametrizations of the total expected background in the double-tag samples. The functional form is the product of Landau and Gaussian functions, $f\left(p_{T}\right) \propto L\left(p_{T}, p_{1}, p_{2}\right) G\left(p_{T}, 30, p_{3}\right)$.

\begin{tabular}{|c|c|c|c|}
\hline \multicolumn{2}{|c|}{ jet multiplicity } & 3 & 4 \\
\hline \hline $\mathrm{CEM}$ & $p_{1}$ & $0.0346 \pm 0.0004$ & $0.0391 \pm 0.0003$ \\
\hline CMUP/CMX & $p_{1}$ & $0.0374 \pm 0.0002$ & $0.0375 \pm 0.0002$ \\
\hline
\end{tabular}

Table 4.7: Parametrizations of the total expected background in the single-tag samples. The functional form is $f\left(p_{T}\right) \propto \exp \left[\left(-p_{1} \cdot p_{T}\right)\right]$.

and the first term is the Landau distribution [53],

$$
L\left(x, p_{1}, p_{2}\right)=\frac{1}{2 \pi i} \int_{\sigma-i \infty}^{\sigma+i \infty} \exp \left[u \ln u+\frac{x-p_{1}}{p_{2}} u\right] d u \text {. }
$$

Here $\sigma$ is any finite real number greater than zero, and $p_{1}$ and $p_{2}$ are the "most probable value" and "width" of the distribution. The Landau distribution is approximated by rational functions [54], as implemented in the CERNLIB software libraries [55].

The results of these fits are listed in Table 4.10 .

\subsection{The dilepton sample}

We include the $t \bar{t}$ dilepton sample, where two charged leptons, two jets, $\mathbb{E}_{T}>25 \mathrm{GeV}$, and $H_{T}>200 \mathrm{GeV}$ are required. The full event selection for this sample is described elsewhere [56]. The total integrated luminosity for this sample is $193 \mathrm{pb}^{-1}$.

\subsubsection{Dilepton background models}

Table 4.11 shows the expected background composition of the $e e, \mu \mu$ and $e \mu$ dilepton samples. For each sample we model the background probability density as a function of charged-lepton $p_{T}$ by assembling histograms representing the contribution of each 


\begin{tabular}{|c|c|c|c|}
\hline & $a_{L 0}$ & $a_{R 0}$ & $a_{L R}$ \\
\hline$C E M$ & $0.790 \pm 0.036$ & $0.977 \pm 0.042$ & $0.809 \pm 0.036$ \\
$C M U P+C M X$ & $0.712 \pm 0.035$ & $0.945 \pm 0.043$ & $0.753 \pm 0.037$ \\
$C E M+C M U P / C M X$ & $0.754 \pm 0.025$ & $0.962 \pm 0.030$ & $0.783 \pm 0.026$ \\
\hline
\end{tabular}

Table 4.8: Acceptance ratios for the lepton +3 jet samples.

\begin{tabular}{|c|c|c|c|}
\hline & $a_{L 0}$ & $a_{R 0}$ & $a_{L R}$ \\
\hline CEM & $0.715 \pm 0.030$ & $1.060 \pm 0.040$ & $0.674 \pm 0.027$ \\
CMUP+CMX & $0.751 \pm 0.034$ & $1.011 \pm 0.043$ & $0.743 \pm 0.033$ \\
CEM+CMUP/CMX & $0.732 \pm 0.022$ & $1.038 \pm 0.029$ & $0.704 \pm 0.021$ \\
\hline
\end{tabular}

Table 4.9: Acceptance ratios for the lepton +4 jet samples.

background process to the overall acceptance. These histograms are weighted according to the background composition estimates given in Table 4.11. We model all but the fake lepton component of the background using Monte Carlo-generated events. The fake lepton background component is predominantly due to $W+$ jets events where the $W$ decays leptonically and one of the light-quark jets manages to pass the lepton ID requirements. We model the fake lepton component using real lepton+jets events which have one jet which could fake a charged lepton.

We parametrize the background probability density by fitting the resulting histograms to the function given in Equation (4.1). The parametrizations of the overall background probability densities are shown in Figure 4.7. In Figure 4.6 we show distribution of charged-lepton $p_{T}$ from the inclusive dilepton sample sample plotted against the total background expectation. Note that the observed $p_{T}$ spectrum is softer than expected; this feature and its impact on the measurement of $F_{0}$ is discussed in Section 7.2.

\begin{tabular}{|c|c|c|c|}
\hline parent of charged-lepton & left-handed $W$ & longitudinal $W$ & right-handed $W$ \\
\hline$p_{1}$ & $28.0 \pm 0.9$ & $41.6 \pm 0.6$ & $63.9 \pm 1.6$ \\
$p_{2}$ & $8.8 \pm 0.4$ & $13.7 \pm 0.6$ & $23.6 \pm 1.1$ \\
$p_{3}$ & $63.0 \pm 3.6$ & $82.1 \pm 3.8$ & $89.0 \pm 3.7$ \\
\hline
\end{tabular}

Table 4.10: Parametrization of the signal PDFs. The functional form is the product of Landau and Gaussian functions, $f\left(p_{T}\right) \propto L\left(p_{T}, p_{1}, p_{2}\right) G\left(p_{T}, 30, p_{3}\right)$ 


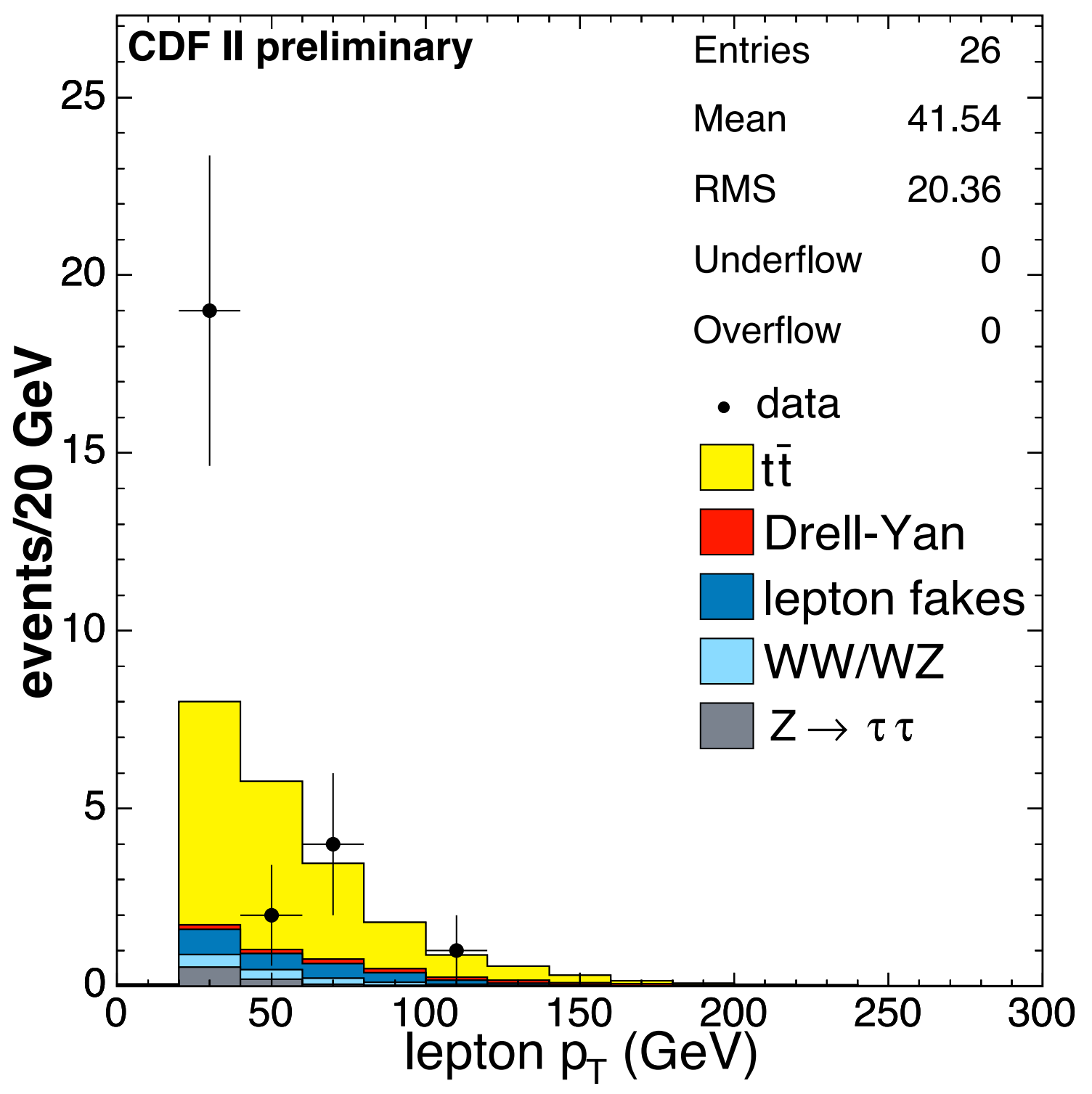

Figure 4.6: Distribution of charged-lepton $p_{T}$ from the inclusive dilepton sample. 


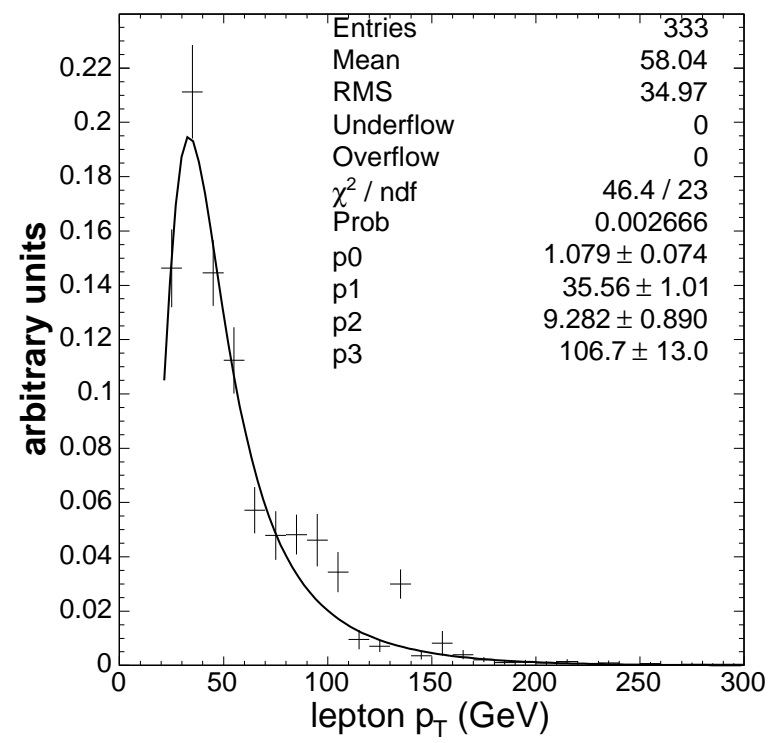

(a)

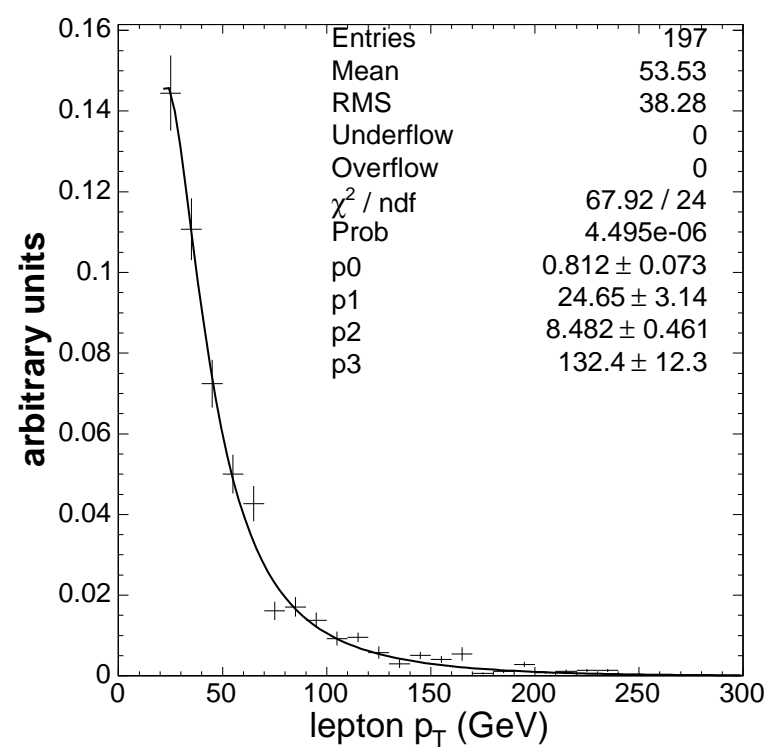

(c)

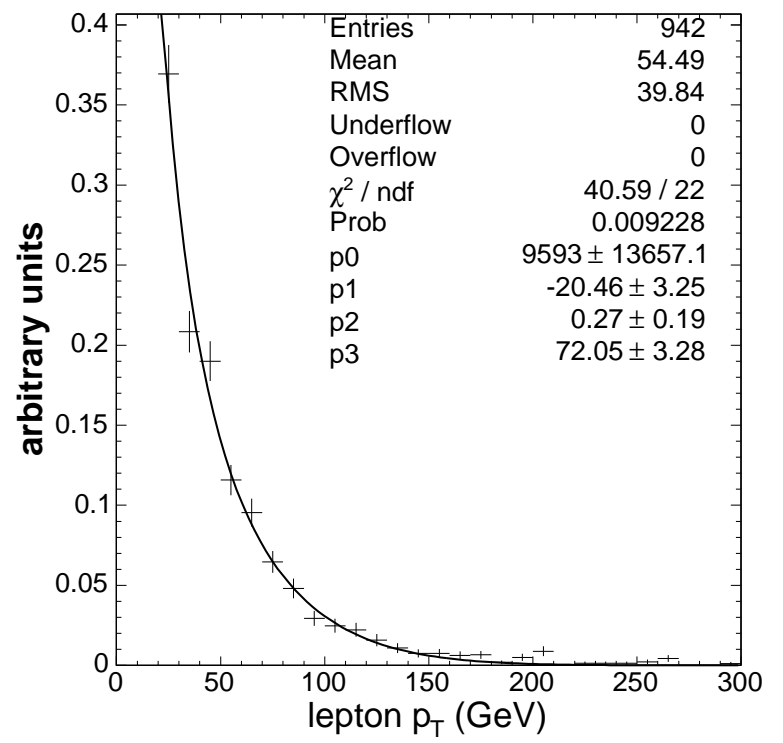

(b)

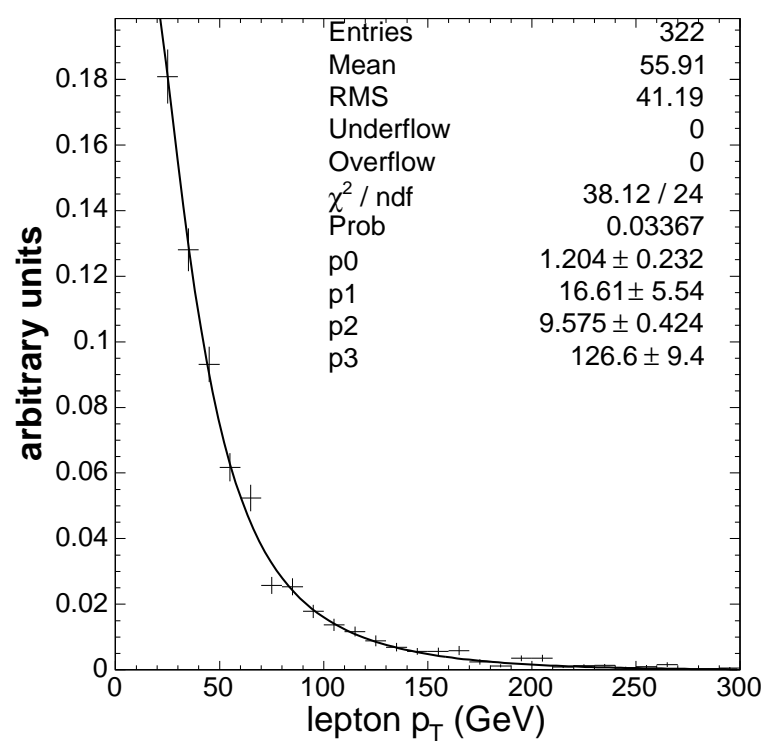

(d)

Figure 4.7: Parametrizations of the total expected backgrounds in the dilepton samples. (a) The ee sample (b) The $e \mu$ sample (c) The $\mu \mu$ sample (d) The $\ell \ell$ sample. 


\begin{tabular}{|c|c|c|c|c|}
\hline event type & $e e$ & $\mu \mu$ & $e \mu$ & $\ell \ell$ \\
\hline$W W / W Z$ & $0.21 \pm 0.06$ & $0.18 \pm 0.05$ & $0.34 \pm 0.10$ & $0.74 \pm 0.21$ \\
Drell-Yan & $0.36 \pm 0.28$ & $0.07 \pm 0.34$ & - & $0.43 \pm 0.44$ \\
$Z \rightarrow \tau \tau$ & $0.09 \pm 0.03$ & $0.11 \pm 0.03$ & $0.22 \pm 0.07$ & $0.42 \pm 0.13$ \\
Fakes & $0.26 \pm 0.11$ & $0.16 \pm 0.07$ & $0.69 \pm 0.28$ & $1.1 \pm 0.45$ \\
\hline Total Background & $0.9 \pm 0.4$ & $0.5 \pm 0.1$ & $1.3 \pm 0.3$ & $2.7 \pm 0.7$ \\
\hline$t \bar{t}(\sigma=6.7 \mathrm{pb})$ & $1.9 \pm 0.3$ & $1.8 \pm 0.3$ & $4.5 \pm 0.6$ & $8.2 \pm 1.1$ \\
\hline Total SM expectation & $2.8 \pm 0.5$ & $2.4 \pm 0.3$ & $5.7 \pm 0.7$ & $10.8 \pm 1.4$ \\
\hline \hline observed & 1 & 3 & 9 & 13 \\
\hline
\end{tabular}

Table 4.11: Background composition estimates for the dilepton samples.

\begin{tabular}{|c|c|c|c|c|}
\hline sample & $e e$ & $\mu \mu$ & $e \mu$ & $\ell \ell$ \\
\hline$A_{00}$ & $0.0024 \pm 0.0002$ & $0.0027 \pm 0.0002$ & $0.0060 \pm 0.0003$ & $0.0112 \pm 0.0004$ \\
$A_{0 L}$ & $0.0019 \pm 0.0001$ & $0.0023 \pm 0.0002$ & $0.0049 \pm 0.0002$ & $0.0091 \pm 0.0003$ \\
$A_{L L}$ & $0.0016 \pm 0.0001$ & $0.0022 \pm 0.0002$ & $0.0040 \pm 0.0002$ & $0.0079 \pm 0.0003$ \\
\hline
\end{tabular}

Table 4.12: Acceptances for the dilepton samples.

\subsubsection{Dilepton acceptance}

We estimate the acceptances given the dilepton event selection for the cases where both $W$ 's from $t \bar{t} \rightarrow$ dileptons are longitudinally polarized, $A_{00}$, one $W$ is longitudinally polarized and one is left-handed, $A_{0 L}$, or both $W^{\prime}$ 's are left handed, $A_{L L}$. To do this it was necessary to re-weight the HERWIG Monte Carlo samples ttopmi-ri according to the angle between the charged lepton and the top quark in the rest-frame of the "second" $W$, since these samples were generated such that the "second" $W$ does not have fixed helicity. These acceptances are given in table 4.12. 


\section{CHAPTER 5}

\section{Method}

We measure the fraction of longitudinal $W$ bosons produced in top-quark decays by analyzing the charged-lepton $P_{T}$ distributions of top-enriched samples. We employ the method of maximum-likelihood: we construct unbinned likelihood functions composed of the data and probability density functions (PDFs) of charged-lepton $P_{T}$ representing the modeled signal and background components of these samples. The fraction of longitudinal $W$ 's is a parameter of these functions; our estimates of $F_{0}$ are those values $\hat{F}_{0}$ which maximize their respective likelihoods.

\subsection{The likelihood function}

We construct an unbinned likelihood function of the form

$$
\mathscr{L}\left(F_{0}, F_{+}, \beta_{1}, \ldots, \beta_{S}\right)=\prod_{s=1}^{S} G\left(\beta_{s} ; \mu_{s}, \sigma_{s}\right) \prod_{i=1}^{N_{s}} P_{s}\left(x_{i} ; F_{0}, F_{+}, \beta_{s}\right) .
$$

Here the first product is over the number of samples, $S$. The second product is over $N_{s}$, the number of reconstructed charged-leptons in sample $s$. The term $G\left(\beta_{s} ; \mu_{s}, \sigma_{s}\right)$ is a Gaussian constraint on $\beta_{s}$, the fraction of events due to background processes in sample $s$. The mean $\mu_{s}$ and width $\sigma_{s}$ of the constraint term describe an a priori estimate of the background content of the sample. The term $P_{s}\left(x_{i} ; F_{0}, F_{+}, \beta_{s}\right)$ is the conditional probability density of observing a charged-lepton in sample $s$ with $P_{T}=x_{i}$, given $F_{0}, F_{R}$ 
and $\beta_{s}$. In this analysis, the fraction of right-handed $W$ 's from top decay is fixed to zero (see Section 1.2.2). We express the per-charged-lepton probability density,

$$
\begin{aligned}
P_{s}\left(x_{i} ; F_{0}, \beta_{s}\right) / \epsilon_{s}\left(x_{i}\right)=\left(1-\beta_{s}\right)[ & F_{0, s}^{\mathrm{obs}}\left(F_{0}\right) P_{s}\left(x_{i} ; \lambda_{W}=0\right) \\
& \left.+\left(1-F_{0, s}^{\mathrm{obs}}\left(F_{0}\right)\right) P_{s}\left(x_{i} ; \lambda_{W}=-1\right)\right] \\
& +\beta_{s} P_{s}\left(x_{i} ; \text { b.g. }\right),
\end{aligned}
$$

where $\epsilon_{s}\left(x_{i}\right)$ is the efficiency for sample $s$ to trigger on a charged-lepton with $P_{T}=x_{i}$. $P_{s}\left(x_{i} ;\right.$ b.g. $)$ is the probability density for charged leptons in sample $s$ with $P_{T}=x_{i}$, which are due to background processes. $P_{s}\left(x_{i} ; \lambda_{W}=-1\right)$ and $P_{s}\left(x_{i} ; \lambda_{W}=0\right)$ are the PDFs for sample $s$ of reconstructed charged-leptons with $P_{T}=x_{i}$ from the decay chain $t \rightarrow W b$, $W \rightarrow \ell \nu_{\ell}$, where the $W$ has helicity $\lambda_{W}=-1$ or $\lambda_{W}=0$, respectively.

In Chapter 4 we describe the construction of the background and signal PDFs for each sub-sample. We also describe parametrizations of the trigger efficiency for these samples.

\subsubsection{Acceptance correction}

The functions $F_{0, s}^{\text {obs }}\left(F_{0}\right)$ in (5.2) serve to correct the bias on imposed by the event selection. For all samples we require that reconstructed charged leptons have $P_{T} \geq 20 \mathrm{GeV}$. Since charged leptons from left-handed $W$ 's have a softer $P_{T}$ distribution than chargedleptons from longitudinally-polarized $W$ 's, the minimum $P_{T}$ requirement introduces a positive bias on $F_{0}$. Here we derive the correction terms.

\section{Acceptance correction for lepton+jets events}

In the channel $t \bar{t} \rightarrow$ lepton+jets, only the helicity of the leptonically decaying $W$ significantly affects the event acceptance; the helicity information of the hadronically decaying $W$ is largely lost. We can separate $n$, the number of charged-leptons arising 
from $t \bar{t}$ production and decay in the lepton+jets channel,

$$
n=n_{-}+n_{0}
$$

where $n_{-}$and $n_{0}$ are the numbers of charged-leptons from left-handed and longitudinal $W$ 's produced in top decays. The observed number of charged-leptons in sample $s$ is

$$
n_{s}^{\mathrm{obs}}=A_{s}^{-} n_{-}+A_{s}^{0} n_{0}
$$

Here $A_{s}^{-}$and $A_{s}^{0}$ are the fractions of charged leptons from left-handed and longitudinal $W$ 's produced in top decays within events that pass the event selection for sample $s$. The true and the observed fractions of longitudinal $W$ 's are

$$
\begin{gathered}
F_{0}=\frac{n_{0}}{n}, \\
F_{0, s}^{\mathrm{obs}}=\frac{A_{s}^{0} n_{0}}{n_{s}^{\mathrm{obs}}} .
\end{gathered}
$$

We can express the observed fraction relative to the true fraction. To ensure that the correction function is well-behaved for $F_{0} \notin[0,1]$, we define

$$
F_{0, s}^{\mathrm{obs}}\left(F_{0}\right)=\left\{\begin{array}{cc}
\left(1+\frac{A_{s}^{-}}{A_{s}^{0}}\left(\frac{1}{F_{0}}-1\right)\right)^{-1} & 0<F_{0} \leq 1 \\
F_{0} & \text { otherwise. }
\end{array}\right.
$$

We estimate the acceptances $A_{s}^{-}$and $A_{s}^{0}$ by counting reconstructed charged leptons whose parent is a $W$ with $\lambda_{W}=-1$ or $\lambda_{W}=0$ in $t \bar{t}$ events, generated by the HERWIG Monte Carlo program, which pass the event selection requirements for sample $s$. The acceptances for each sub-sample are given in Chapter 4.

\section{Acceptance correction for dilepton events}

In the channel $t \bar{t} \rightarrow$ dileptons, the helicity of both $W^{\prime}$ 's significantly affect the event acceptance. We separate the number of $t \bar{t}$ events

$$
n_{t \bar{t}}=n_{00}+n_{-0}+n_{--}
$$


where $n_{00}, n_{0-}$, and $n_{--}$are the numbers of $t \bar{t}$ events where both $W$ 's have longitudinal polarization, one $W$ has longitudinal and one has left-handed polarization, and both $W$ s have left-handed polarization, respectively. The observed number of $t \bar{t}$ events in sample $s$ is then

$$
n_{t \bar{t}, s}^{\mathrm{obs}}=A_{s}^{00} n_{00}+A_{s}^{0-} n_{0-}+A_{s}^{--} n_{--} .
$$

Here, $A_{s}^{00}, A_{s}^{0-}$, and $A_{s}^{--}$are the acceptances for events where both $W^{\prime}$ 's have longitudinal polarization, one $W$ has longitudinal and one has left-handed polarization, and both $W$ 's have left-handed polarization, respectively. The true and observed fractions of longitudinally polarized $W$ 's from top decay are

$$
\begin{gathered}
F_{0}=\frac{2 n_{00}+n_{0-}}{2 n_{t \bar{t}}}, \\
F_{0, s}^{\mathrm{obs}}=\frac{2 A_{s}^{00} n_{00}+A_{s}^{0-} n_{0-}}{2 n_{t \bar{t}, s}^{\mathrm{obs}}} .
\end{gathered}
$$

Assuming the helicities of the $W$ 's in the event are uncorrelated,

$$
\begin{aligned}
\frac{n_{00}}{n_{t \bar{t}}} & =F_{0}^{2}, \\
\frac{n_{0-}}{n_{t \bar{t}}} & =2 F_{0}\left(1-F_{0}\right), \\
\frac{n_{--}}{n_{t \bar{t}}} & =\left(1-F_{0}\right)^{2} .
\end{aligned}
$$

We express the observed fraction as a function of the true fraction,

$$
F_{0, s}^{\mathrm{obs}}\left(F_{0}\right)=\left\{\begin{array}{cc}
\frac{2 A_{00} F_{0}^{2}+2 A_{0-} F_{0}\left(1-F_{0}\right)}{2\left(A_{00} F_{0}^{2}+2 A_{0-} F_{0}\left(1-F_{0}\right)+A_{--}\left(1-F_{0}\right)^{2}\right)} & F_{0} \in[0,1] \\
F_{0} & \text { otherwise. }
\end{array}\right.
$$

\subsubsection{Trigger correction}

We correct for inefficiency in the trigger by weighting the per-charged-lepton probability 5.2 by the expected efficiency to trigger on such a lepton. In dilepton events where 


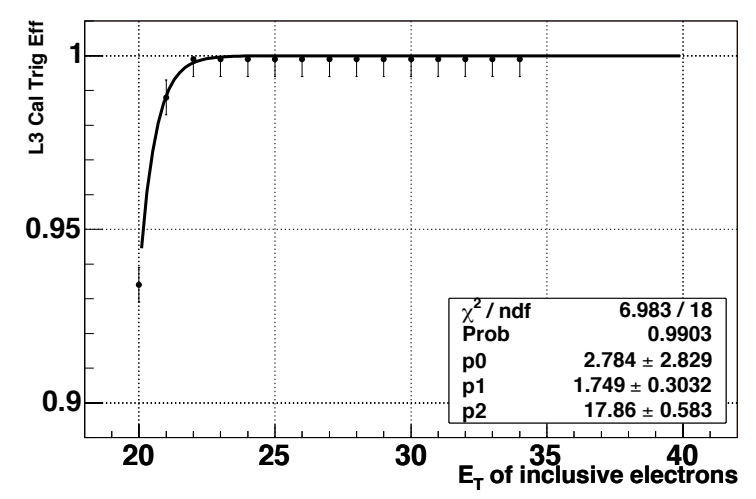

Figure 5.1: Parametrization of the efficiency to trigger on a central electron as a function of the electron $E_{T}$.

both leptons are consistent with the event triggers, we apply the appropriate corrections to each lepton.

We use the parametrization of the L3_CEM_18 trigger efficiency as a function of electron $E_{T}[57]$ to weight CEM electrons. We use the parametrization of the MET_PEM trigger efficiency as a function of electron $E_{T}[58]$ to weight triggered plug electrons in the dilepton sample. We apply no correction for the MUON_CMUP18 and MUON_CMX18 triggers, as there is no apparent $p_{T}$ dependence for muons with track $p_{T}>20 \mathrm{GeV}$. Figure 5.1 shows the efficiency to trigger on a central electron as a function of the electron $E_{T}$. Figure 5.2 shows the efficiency to trigger on a plug electron as a function of the electron $E_{T}$.

\subsection{Minimization procedure}

Our estimates of $F_{0}$ and $\beta_{s}$ (labeled $\hat{F}_{0}$ and $\hat{\beta}_{s}$ ) are the values of these parameters which maximize Equation (5.1). We refer to these as Maximum Likelihood Estimates (MLEs). We determine the MLEs in the usual way, by finding those values which minimize $-\log \mathscr{L}$. To do this we calculate numerically

$$
-\log \left(\mathscr{L}\left(F_{0}, F_{+}, \beta_{1}, \ldots, \beta_{S}\right)\right)=\sum_{s=1}^{S} \frac{\left(\beta_{s}-\mu_{s}\right)^{2}}{2 \sigma_{s}^{2}}-\sum_{i=1}^{N_{s}} \log \left(P_{s}\left(x_{i} ; F_{0}, \beta_{s}\right)\right)+\text { constant }
$$




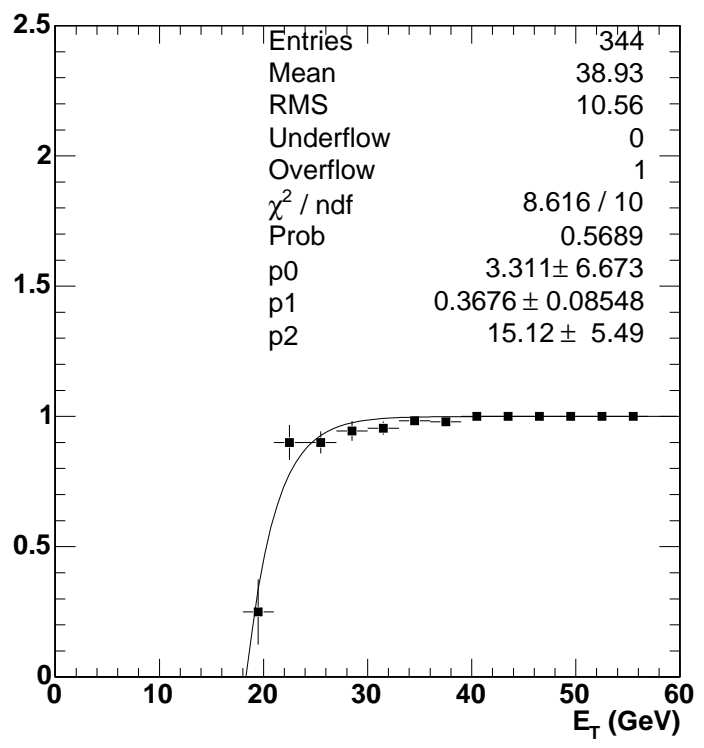

Figure 5.2: Parametrization of the efficiency to trigger on a plug electron as a function of the electron $E_{T}$.

We calculate (5.16) for all values of the parameters $F_{0}$ and $\beta_{s}$ as a double-precision floating point number, in accordance with IEEE standard 754 [59]. For $F_{0}$ or $\beta_{s} \notin[0,1]$, $P_{s}\left(x_{i} ; F_{0}, \beta_{s}\right)$ can take on values less than or equal to zero. When this happens, we add "positive infinity" to Eq. 5.16, following the IEEE standard. In this way we avoid "unmathematical" regions of the likelihood function and we preserve its normalization. We then use the MIGRAD algorithm of the MINUIT [60] software package to scan over the parameters $F_{0}$ and $\beta_{s}$ and find the MLEs $\hat{F}_{0}$ and $\hat{\beta}_{s}$. 


\section{CHAPTER 6}

\section{Estimates of statistical and systematic uncertainty}

The true fraction $F_{0}$ is a real number defined within the interval

$$
F_{0} \in[0,1]
$$

However, as a parameter of the likelihood function (5.1), we do not restrict $F_{0}$ to this range. Doing so would introduce either a positive bias in the case where the true value is close to zero, or a negative bias in the case where the true value is close to one. In order to make a statement about the observed value of $F_{0}$ which is consistent with (6.1), we employ the method of Feldman and Cousins [61], a variant of the classical Neyman method.

\subsection{The Feldman-Cousins method}

By construction, the Feldman-Cousins (FC) method always produces intervals within the defined interval and gives the proper coverage in ensemble for all values of the true parameter. The method also makes a natural transition from situations where it is appropriate to quote an upper or lower limit rather than a central interval. These properties are desirable since we are measuring a parameter to a precision similar to the breadth

of its defined range. We apply the FC method to estimate central $68 \%$ CL intervals and $95 \%$ CL limits. 
For each analysis we produce a large ensemble of Monte Carlo pseudo-experiments which we use as a model of the experimental resolution and bias. Each pseudo-experiment consists of background events generated in accordance with expected background content of each sample, and signal events generated in accordance with a fixed value of $F_{0}$. These pseudo-experiments fully describe our expectation of the experimental circumstance for arbitrary values of $F_{0}$.

For each pseudo-experiment in the ensemble, the value of the true parameter $F_{0}$ is selected at random from a uniform distribution within the interval $[0,1]$. From the ensemble we determine the distribution of the measured parameter $\hat{F}_{0}$ for all possible values of the true parameter. We then construct a parametrization of this distribution called the resolution function $P\left(\hat{F}_{0} ; F_{0}\right)$, i.e. the probability density of $\hat{F}_{0}$ given $F_{0}$.

Suppose we are measuring a single parameter $\mu \in[a, b]$ and we have a reliable estimate of the resolution function $P(\hat{\mu} ; \mu)$. The FC method of interval estimation at the $(100 \times \alpha) \%$ confidence level is as follows. We define an ordering rule

$$
R(\hat{\mu})=\frac{P(\hat{\mu} ; \mu)}{P\left(\hat{\mu} ; \mu_{\text {best }}\right)},
$$

where

$$
\mu_{\text {best }}= \begin{cases}a & \hat{\mu}<a \\ \hat{\mu} & a \leq \hat{\mu} \leq b . \\ b & \hat{\mu}>b\end{cases}
$$

For each value of $\mu$, we finds the values $x_{1}$ and $x_{2}$ such that

$$
R\left(x_{1}\right)=R\left(x_{2}\right),
$$

and

$$
\int_{x_{1}}^{x_{2}} P(x ; \mu) d x=\alpha .
$$




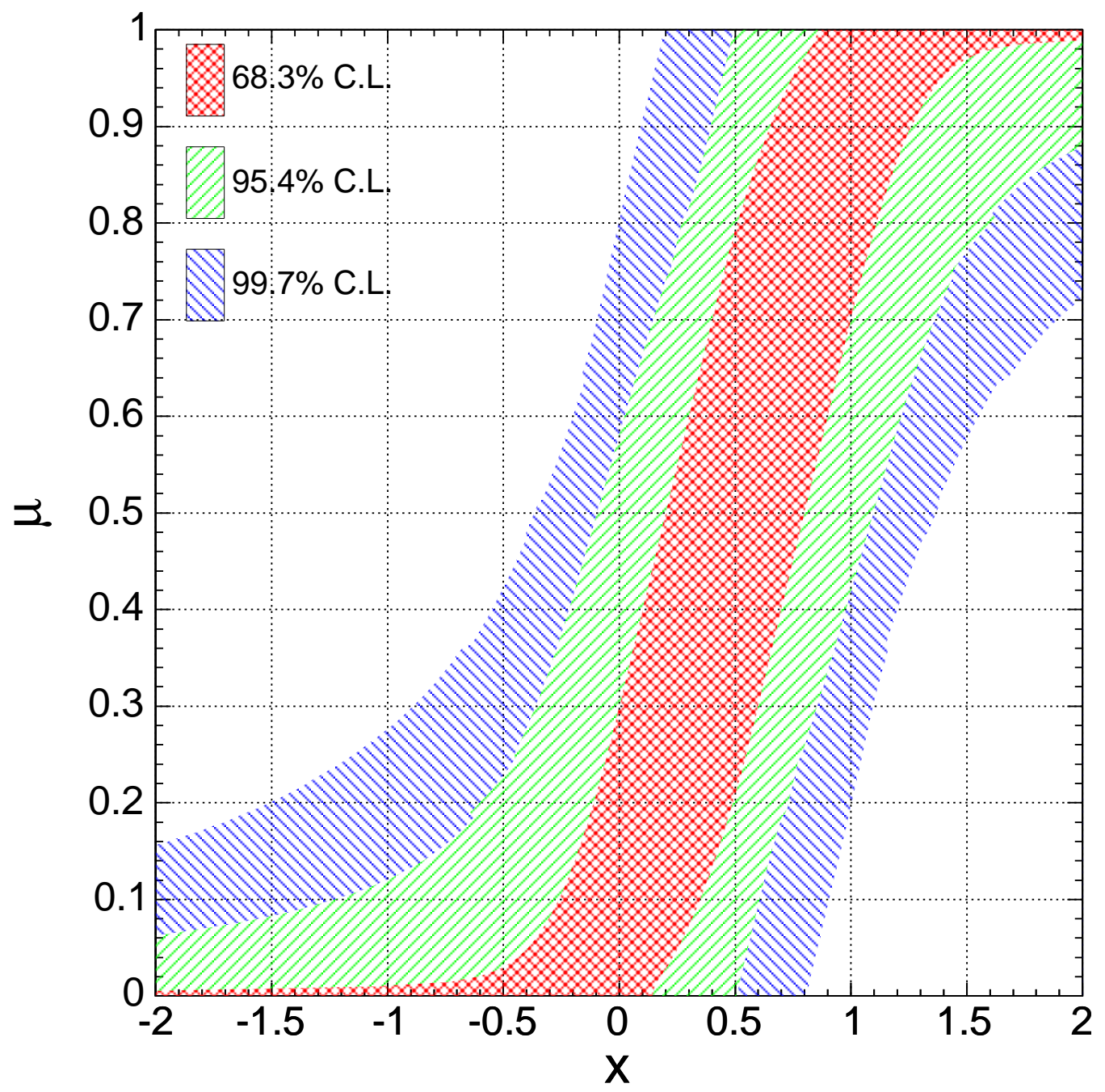

Figure 6.1: Toy Feldman-Cousins confidence belts at the $68.3 \%, 95.4 \%$ and $99.7 \%$ confidence levels for measurement of a parameter $\mu \in[0,1]$ with the experimental resolution function $P(\hat{\mu} ; \mu)=G(\hat{\mu} ; \mu, 0.3)$.

The $(100 \times \alpha) \%$ confidence interval for a measurement $\hat{\mu}$ is the union for all values of $\mu$ where $\hat{\mu} \in\left[x_{1}, x_{2}\right]$.

For example, Figure 6.1 shows the 1,2 and $3 \sigma$ confidence belts for the case where we measure a parameter $\mu \in[0,1]$ with a Gaussian resolution function with mean $m(\mu)=\mu$ (i.e. the measurement is unbiased) and constant width, $\sigma=0.3$. If an experiment yields the result $\hat{\mu}=0.50$, we could make the statement $\mu=0.50 \pm 0.29$ at the $68 \%$ confidence level; we would not be able to quote an upper or lower limit at the $95 \%$ or $99 \%$ confidence 
levels. If an experiment yields the unlikely result $\hat{\mu}=-1.5$, we could make the statement $\mu<0.08$ at the $95 \%$ confidence level and $\mu<0.20$ at the $99 \%$ confidence level.

\subsubsection{Calculating Feldman-Cousins confidence intervals}

It is difficult to compute FC confidence intervals analytically; we take the numerical approach. We assume the resolution function is for all values of the true parameter a Gaussian with width $\sigma(\mu)$ and mean $a<m(\mu)<b$. We step through values of the true parameter with a step-size much smaller than the width of the resolution function. For each value of the true parameter, we find analytically the values $x_{1}^{\prime}$ and $x_{2}^{\prime} \forall R \in(0,1]$ which satisfy Eq. 6.4. We can express the area under the resolution function as a function of $R$

$$
\int_{x_{1}^{\prime}}^{x_{2}^{\prime}} P(x ; \mu) d x=f\left(x_{1}^{\prime}, x_{2}^{\prime}\right)=A(R) .
$$

Using MIGRAD, we allow $R$ to vary, and find the value $R_{\text {min }}$ which minimizes the quantity

$$
\left(\int_{x_{1}^{\prime}}^{x_{2}^{\prime}} P(x ; \mu) d x-\alpha\right)^{2}=(A(R)-\alpha)^{2} .
$$

From $R_{\min }$ we find those values $x_{1}$ and $x_{2}$ which simultaneously satisfy Eqns. 6.4 and 6.5.

\subsubsection{Feldman Cousins resolution functions}

To construct confidence belts according to the Feldman Cousins method, it is necessary to develop an understanding of experimental resolution and bias for all possible values of the parameter(s) to be measured. We establish this understanding by casting many pseudo-experiments, each consistent with our expected sample size and background composition. In these pseudo-experiments we allow the true parameter $F_{0}$ to vary uniformly between 0 and 1 and generate events accordingly. We fit these pseudo-data according

to the procedure described in Chapter 5 to obtain the MLE $\hat{F}_{0}$. We observe that the distribution of MLEs is Gaussian for constant $F_{0}$. We construct parametrizations of the 
mean and width of the distribution of MLEs as a function of the true parameter, i.e. $m\left(F_{0}\right)$ and $\sigma\left(F_{0}\right)$. We assemble these to form the resolution function

$$
P\left(\hat{F}_{0} ; F_{0}\right)=G\left(\hat{F}_{0} ; m\left(F_{0}\right), \sigma\left(F_{0}\right)\right)
$$

\subsection{Resolution function for the lepton+jets analysis}

Figure 6.2(a) shows a parametrization of the mean of the distribution of MLEs as a function of the true parameter $F_{0}$ for pseudo-experiments where only the lepton+jets samples are included. Each bin along the $x$-axis contains the MLEs $\hat{F}_{0}$ from 1000 pseudoexperiments where $F_{0}$ varies uniformly within the range of the bin. The values along the $y$-axis are the means of Gaussian fits to the distribution of $\hat{F}_{0}$ in each bin. Based on this ensemble of pseudo-experiments, we conclude that our procedure for estimating $F_{0}$ in the lepton + jets analysis is unbiased.

Figure 6.2(b) shows a parametrization of the width of the distribution of MLEs as a function of the true parameter $F_{0}$ for pseudo-experiments where only the lepton+jets samples are included. Each bin along the $x$-axis contains the MLEs $\hat{F}_{0}$ from 1000 pseudoexperiments where $F_{0}$ varies uniformly within the range of the bin. The values along the $y$-axis are the widths of Gaussian fits to the distributions of $\hat{F}_{0}$ in each bin.

\subsection{Resolution function for the dilepton analysis}

Figure 6.3(a) shows a parametrization of the mean of the distribution of MLEs as a function of the true parameter $F_{0}$ for pseudo-experiments where only the dilepton samples are included. Each bin along the $x$-axis contains the MLEs $\hat{F}_{0}$ from 1000 pseudoexperiments where $F_{0}$ varies uniformly within the range of the bin. The values along the $y$-axis are the means of Gaussian fits to the distribution of $\hat{F}_{0}$ in each bin. Based on this ensemble of pseudo-experiments, we conclude that our procedure for estimating $F_{0}$ in the 


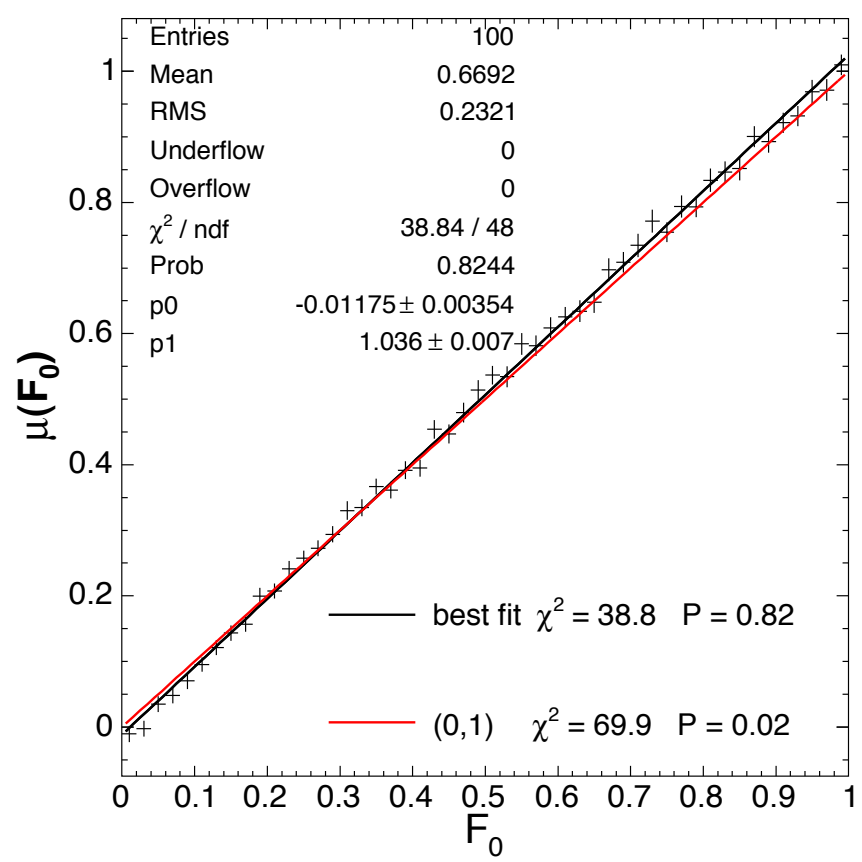

(a) Parameterization of $m\left(F_{0}\right)$ for pseudo-experiments where only the lepton+jets samples are included. The functional form is a first-order polynomial. The black curve is the best fit, the red curve has zero intercept and unit slope.

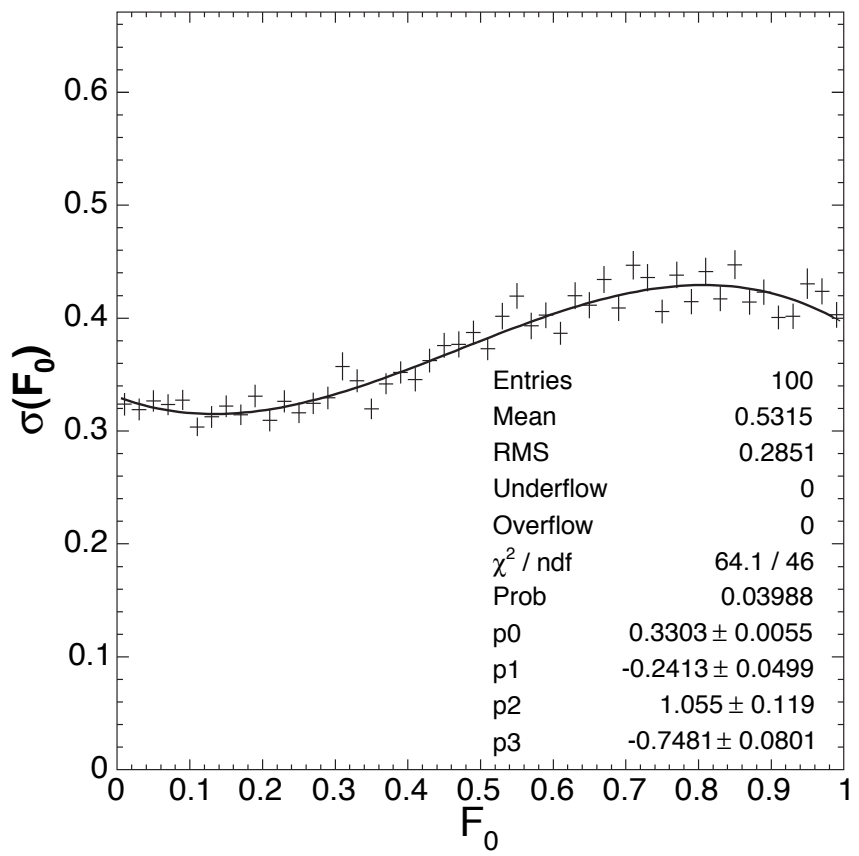

(b) Parameterization of $\sigma\left(F_{0}\right)$ for pseudo-experiments where only the lepton+jets samples are included. The functional form is a third-order polynomial.

Figure 6.2: Ensemble tests for the lepton+jets only analysis. 


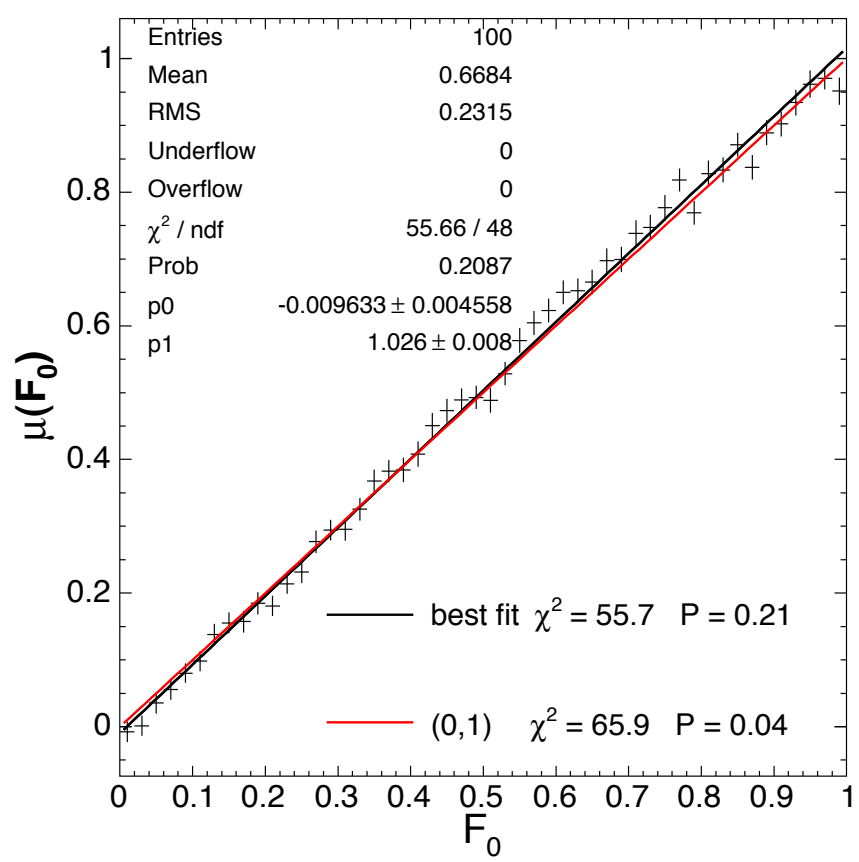

(a) Parameterization of $m\left(F_{0}\right)$ for pseudo-experiments where only the dilepton samples are included. The functional form is a firstorder polynomial. The black curve is the best fit, the red curve is a first-order polynomial with zero intercept and unit slope.

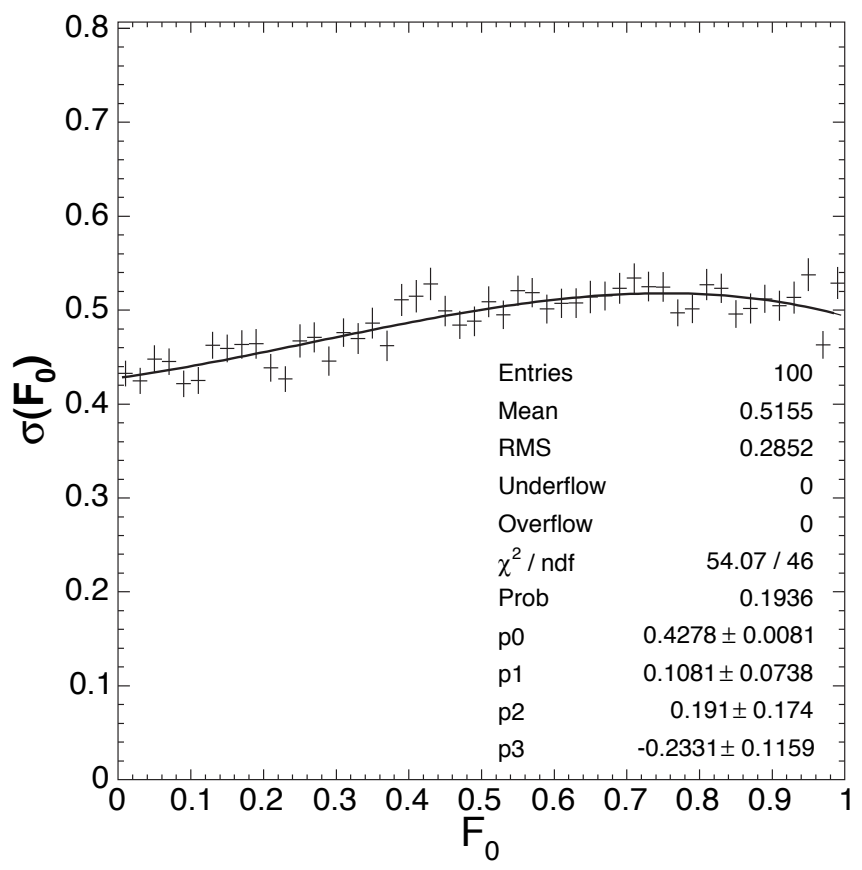

(b) Parameterization of $\sigma\left(F_{0}\right)$ for pseudo-experiments where only the dilepton samples are included. The functional form is that of a third-order polynomial.

Figure 6.3: Ensemble tests for the dilepton-only analysis. 
dilepton analysis is unbiased.

Figure 6.3(b) shows a parametrization of the width of the distribution of MLEs as a function of the true parameter $F_{0}$ for pseudo-experiments where only the dilepton samples are included. Each bin along the $x$-axis contains the MLEs $\hat{F}_{0}$ from 1000 pseudoexperiments where $F_{0}$ varies uniformly within the range of the bin. The values along the $y$-axis are the widths of Gaussian fits to the distributions of $\hat{F}_{0}$ in each bin.

\subsection{Resolution function for the combined analysis}

Figure 6.4(a) shows a parametrization of the mean of the distribution of MLEs as a function of the true parameter $F_{0}$ for pseudo-experiments where only the lepton + jets and dilepton samples are included. Each bin along the $x$-axis contains the MLEs $\hat{F}_{0}$ from 1000 pseudo-experiments where $F_{0}$ varies uniformly within the range of the bin. The values along the $y$-axis are the means of Gaussian fits to the distribution of $\hat{F}_{0}$ in each bin. Based on this ensemble of pseudo-experiments, we conclude that our procedure for estimating $F_{0}$ in the combined analysis is unbiased.

Figure 6.4(b) shows a parametrization of the width of the distribution of MLEs as a function of the true parameter $F_{0}$ for pseudo-experiments where the lepton+jets and dilepton samples are included. Each bin along the $x$-axis contains the MLEs $\hat{F}_{0}$ from 1000 pseudo-experiments where $F_{0}$ varies uniformly within the range of the bin. The values along the $y$-axis are the widths of Gaussian fits to the distributions of $\hat{F}_{0}$ in each bin.

\subsection{Estimates of systematic uncertainty}

We incorporate systematic uncertainties with the Feldman-Cousins method by modifying the resolution function such that the statistical uncertainty $\sigma\left(F_{0}\right)$ is added in quadra- 


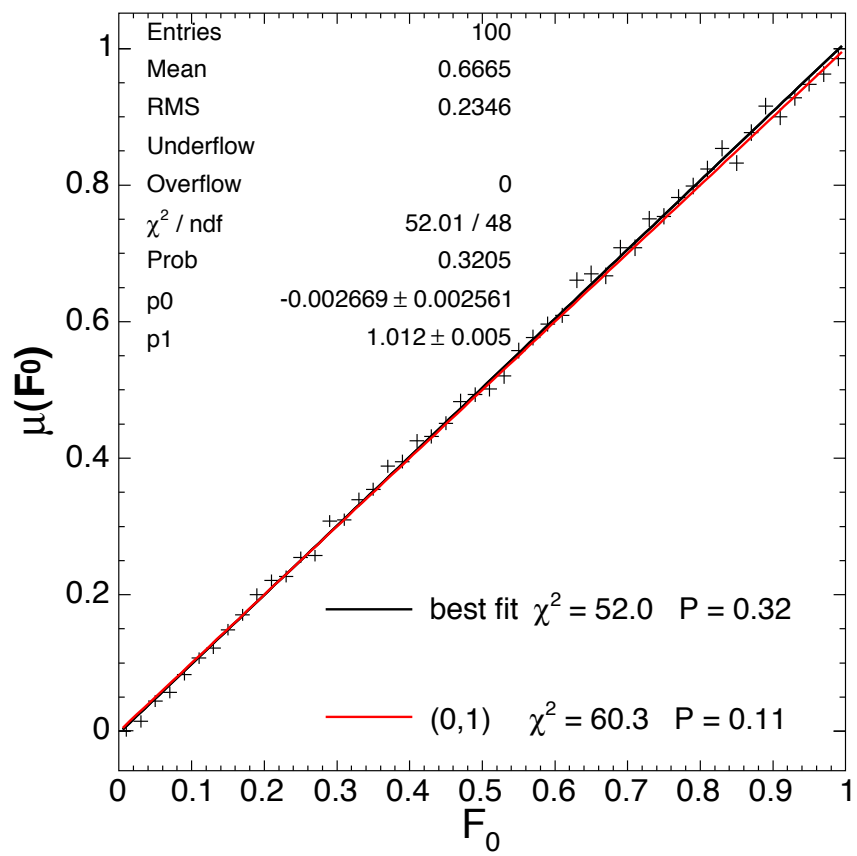

(a) Parameterization of $m\left(F_{0}\right)$ for pseudo-experiments where the lepton+jets and dilepton samples are included. The functional form is a first-order polynomial. The black curve is the best fit, the red curve is a first-order polynomial with zero intercept and unit slope.

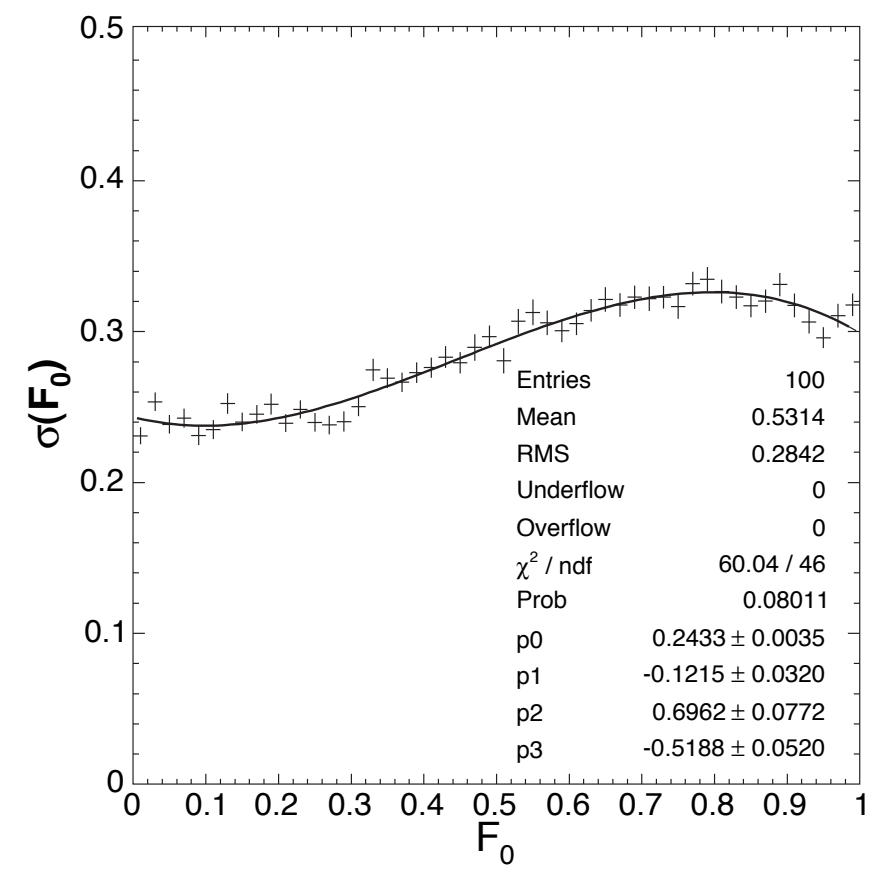

(b) Parameterization of $\sigma\left(F_{0}\right)$ for pseudo-experiments where the lepton+jets and dilepton samples are included. The functional form is a third-order polynomial.

Figure 6.4: Ensemble tests for the combined analysis. 
ture with our estimate of the systematic uncertainty $\sigma_{\text {syst. }}$,

$$
P\left(\hat{F}_{0} ; F_{0}\right)=G\left(\hat{F}_{0} ; \mu\left(F_{0}\right), \sigma\left(F_{0}\right) \oplus \sigma_{\text {syst. }}\right)
$$

Systematic uncertainties on $F_{0}$ arise from two basic sources: uncertainties inherent to the models of the charged-lepton $p_{T}$ distribution of the signal, and uncertainties inherent to the models of the charged-lepton $p_{T}$ distributions of the background. The latter include uncertainties on estimates of the rates of various contributions to the background, and uncertainties in modeling of the charged-lepton $p_{T}$ distributions of each background component. We consider the effect of the following sources of uncertainty:

- Modeling of top signal:

- top-quark mass

- uncertain aspects of models of initial- and final-state radiation

- choice of parton density functions

- MC statistics

- acceptance correction

- trigger correction

- Modeling of the background:

- normalization of background components

- shapes of background components

All systematic uncertainties are determined by casting pseudo-experiments. We carry out a procedure similar to the one used to estimate the resolution functions, described in section 6.1.2. However, in these cases we vary the probability density functions used to generate the pseudo-data by varying each uncertain aspect of our model within the limits 
of those uncertainties. We then fit the varied pseudo-data using our default signal and background models and acceptance corrections. We compare the mean of the distribution of measured $\hat{F}_{0}$ for the modified pseudo-experiments with the mean from the default experiments; we take the maximum separation in means as the systematic due to the uncertainty on the varied parameter. We take the quadrature sum of each variation to be our total estimate of systematic uncertainty.

\subsubsection{Systematics from the signal models}

We illustrate the process of estimating systematic uncertainty by casting pseudoexperiments by examining the uncertainty due to our limited knowledge of the top-quark mass. To test our sensitivity to the $5 \mathrm{GeV}$ uncertainty on the top mass, we compare the results obtained from pseudo-experiments where the signal model is consistent with a $175 \mathrm{GeV}$ top-quark to the results of pseudo-experiments where a $170 \mathrm{GeV}$ or $180 \mathrm{GeV}$ top-quark is assumed. The maximum separation of means for these three cases is 0.11 (absolute); we take this as our systematic uncertainty due to uncertainty on the top-quark mass.

To test our sensitivity to initial- and final-state radiation, we throw pseudo-experiments using a $t \bar{t}$ sample where initial-state radiation is turned off (ttop0e) and a sample generated with PYTHIA Tune B (ttop5e). Final-state radiation systematics are tested with a sample that uses a low value of $\alpha_{s}$ to reduce FSR (ttop6e). These give shifts in mean $F_{0}$ of $0.02,0.03$ and 0.04 respectively for the combined measurement. As turning radiation off completely is probably an overestimate, we take half of the first uncertainty and add it in quadrature with the others for a total uncertainty of 0.05 due to uncertainty on modeling of radiation.

The charged-lepton $p_{T}$ spectrum from top-quark decay is sensitive to the parton dis- 
tributions used in the simulation. We compare pseudo-experiments thrown assuming CTEQ5L PDFs (ttopei) and MRST72 and MRST75 PDFs (ttop3e and ttop4e). We find the separation in means to be 0.03 in the combined samples, and take this as our estimate of systematic uncertainty due to choice of PDFs.

Another uncertainty in our model of the top-quark decay is due to our parametrization of the charged-lepton $p_{T}$ spectrum. We estimate the systematic uncertainty due to limited Monte Carlo statistics by generating pseudo-experiments. We take the histograms of charged-lepton $p_{T}$ used in the parametrization of the signal shapes and Poisson-fluctuate the contents of each bin, using the actual bin content as the mean. We fit the fluctuated histogram to obtain new a new parametrization of the signal. We then throw pseudoexperiments using the fluctuated signal model and default background model, and fit using the default signal model. We create 500 new sets of templates in this way; for each set of templates, we throw 1000 pseudo-experiments. We take the mean of the distribution of measured $F_{0}$ for each set of templates as the result of each fluctuation. We then plot the distribution of means, and take its width as our estimate of systematic uncertainty. In the combined measurement this is 0.01 .

\subsubsection{Systematics from the background models}

The systematics due to uncertainties in the background models can be broken into two categories - those due to uncertainty on the normalization of the background components, and those due to uncertainties on the parametrization of the background distributions. We begin by studying the effects of varying the background component normalization.

For the dilepton sample we assume that the normalizations of each of the background components are uncorrelated, and we fluctuate each one by its uncertainties. We throw pseudo-experiments for each new background configuration, and take the maximum sep- 
aration in means as an estimate of systematic uncertainty due to uncertainty on dilepton background normalization. In the dilepton-only measurement this uncertainty is 0.04 . In the combined analysis, this uncertainty is 0.02 .

Estimating the systematic due to uncertainties on the background normalizations in the lepton+jets samples is more subtle, because various components of the background are correlated with each other. The correlated components must be varied simultaneously, and the resulting changes must then be propagated through the sample-composition estimates. We vary the QCD normalization within its quoted uncertainties; this affects the predicted amounts of $W$ plus heavy flavor events. The amount of $W$ plus heavy flavor in the sample depends on the heavy-flavor fractions; we vary them within their quoted uncertainties. The various backgrounds estimated through simulation samples (dibosons, single top, $Z \rightarrow \tau \tau)$ are correlated through their dependence on the scale factor. We vary the scale factor within its uncertainties, and propagate the results through the determination of the sample composition. The mistag rate is also varied independently. The differences in mean $\hat{F}_{0}$ from pseudo-experiments representing the lepton + jets measurement with each of these variations are given in Table 6.1. We add these in quadrature to obtain an overall systematic of 0.11 from uncertainties on lepton+jets background normalization in the lepton+jets measurement. We estimate a systematic of 0.10 due to uncertainties on lepton+jets background normalization in the combined measurement.

\begin{tabular}{|l|c|}
\hline Factor varied & $\Delta \hat{F}_{0}$ \\
\hline QCD non- $W$ & 0.09 \\
Heavy-flavor fractions & 0.05 \\
Scale factor & 0.02 \\
Mistags & 0.01 \\
\hline Total & 0.11 \\
\hline
\end{tabular}

Table 6.1: Shifts in $\hat{F}_{0}$ due to variations in the normalizations of lepton+jets backgrounds in the lepton+jets measurement. 


\begin{tabular}{|l|c|}
\hline Factor varied & $\Delta \hat{F}_{0}$ \\
\hline QCD non- $W$ & 0.09 \\
Heavy-flavor fractions & 0.04 \\
Scale factor & 0.01 \\
Mistags & 0.01 \\
\hline Total & 0.10 \\
\hline
\end{tabular}

Table 6.2: Shifts in $\hat{F}_{0}$ due to variations in the normalizations of lepton + jets backgrounds in the combined measurement.

We estimate the systematic due to uncertainties in the background shape model in the dilepton measurement by fluctuating each parameter of the three background parametrizations within its uncertainties. We cast pseudo-experiments with the fluctuated background models and take the maximum separation in means as the systematic due to shape uncertainties. In the dilepton sample this systematic is 0.02 .

For the lepton+jets samples, we vary the shapes of two of the background components to get to represent the effects of shape uncertainties. We change the non- $W$ shape by replacing our usual model of non-isolated leptons with a spectrum determined through the angular correlations method described in CDF 6559 [62]. This is believed to give a sample that is more enriched in real QCD events, and depleted of $W$ production. We also try several different models of $W b \bar{b}$ production, using simulation samples made with different $Q^{2}$ scales (atop0s, atop1s, atop2s, atop3s). We take the systematic to be 0.03 in the lepton+jets measurement and 0.02 in the combined measurement.

We estimate the systematic due to uncertainties in the acceptance and trigger corrections by fluctuating each parameter of these corrections within their uncertainties, casting pseudo-experiments for each variation and taking the maximum separation in $\hat{F}_{0}$ due to these variations as the overall systematic. In the combined measurement we estimate the systematic due to the acceptance correction to be 0.01. In the combined measurement we also estimate the systematic due to the trigger correction to be 0.01 . 
Summaries of all the systematic uncertainties are given in tables $6.3,6.4$ and 6.5 .

\begin{tabular}{|l|c|}
\hline Source & $\Delta \hat{F}_{0}$ \\
\hline background normalization & 0.11 \\
top mass uncertainty & 0.09 \\
ISR/FSR & 0.04 \\
PDF uncertainty & 0.03 \\
shape uncertainty & 0.03 \\
MC statistics & 0.01 \\
acceptance correction & 0.01 \\
trigger correction & 0.01 \\
\hline total & 0.17 \\
\hline
\end{tabular}

Table 6.3: Estimates of systematic uncertainty in the lepton+jets analysis.

\begin{tabular}{|l|l|}
\hline Source & $\Delta \hat{F}_{0}$ \\
\hline top mass uncertainty & 0.12 \\
ISR/FSR & 0.06 \\
background normalization & 0.04 \\
PDF uncertainty & 0.04 \\
acceptance correction & 0.03 \\
shape uncertainty & 0.02 \\
trigger correction & 0.02 \\
MC statistics & 0.01 \\
\hline total & 0.16 \\
\hline
\end{tabular}

Table 6.4: Estimates of systematic uncertainty in the dilepton analysis. 


\begin{tabular}{|l|l|}
\hline Source & $\Delta \hat{F}_{0}$ \\
\hline top mass uncertainty & 0.11 \\
l+jets bg. normalization & 0.10 \\
ISR/FSR & 0.05 \\
PDF uncertainty & 0.03 \\
dilepton bg. normalization & 0.02 \\
l+jets shape uncertainty & 0.02 \\
acceptance correction & 0.02 \\
trigger correction & 0.02 \\
dilepton shape uncertainty & 0.01 \\
MC statistics & 0.01 \\
\hline total & 0.17 \\
\hline
\end{tabular}

Table 6.5: Estimates of systematic uncertainty in the combined analysis 


\section{CHAPTER 7}

\section{Results}

We apply the parameter estimation procedure described in Chapter 5 along with the sample composition estimates of Chapter 4 to the data. We first consider the lepton+jets and dilepton samples separately. We then find the value $\hat{F}_{0}$ which maximizes the joint dilepton and lepton+jets likelihood.

\subsection{Result from the lepton+jets analysis}

Here we include in the likelihood function only the eight lepton + jets samples. Using MIGRAD we find the MLE for this sample, $\hat{F}_{0}=0.88$. Figure 7.1 shows the projection of $-\log (\mathscr{L})$ along the $F_{0}$ axis, where the background fractions for each sub-sample are fixed to those values which minimize $-\log (\mathscr{L})$ when $F_{0}=0.88$. Figure 7.1 also shows the distribution of charged-lepton $p_{T}$ data for the eight lepton+jets samples overlaid with the total signal and background PDFs normalized according to their MLEs.

Using the resolution function derived in section 6.1.2 for the lepton+jets only measurement, and the estimate of systematic uncertainty from table 6.3 we construct Feldman Cousins confidence belts at the $68.3 \%, 95.4 \%$ and $99.7 \%$ confidence levels. These belts are plotted in figure 7.2, along with MLE for this measurement. From this construction

we find $F_{0}=0.88_{-0.47}^{+0.12}$ (stat. + syst.) and $F_{0}>0.24 @ 95 \%$ CL in the lepton+jets only 
measurement. This result is consistent with the SM prediction.

\subsection{Result from the dilepton analysis}

Here we include in the likelihood function only the three dilepton sub-samples. Using MIGRAD, we find the MLE for this sample, $\hat{F}_{0}=-0.54$. Figure 7.3 shows the projection of $-\log (\mathscr{L})$ along the $F_{0}$ axis, where the background fractions for each sub-sample are fixed to those values which minimize $-\log (\mathscr{L})$ when $F_{0}=-0.54$. Figure 7.3 also shows the distribution of charged-lepton $p_{T}$ data for the three dilepton samples overlaid with the total signal and background PDFs normalized according to their MLEs. In this case, the distribution of charged-lepton $p_{T}$ from the data is softer than any component of the signal or background in our model. As a consequence, the longitudinal component, which has a harder $p_{T}$ distribution than the left-handed component, is forced to be negative to fit the data. However, we can make a statement about the true value of $F_{0}$ by applying the Feldman Cousins method.

Using the resolution function derived in section 6.1 .2 for the lepton+jets only measurement, and the estimate of systematic uncertainty from table 6.4 we construct Feldman Cousins confidence belts at the $68.3 \%, 95.4 \%$ and $99.7 \%$ confidence levels. These belts are plotted in figure 7.4, along with MLE for this measurement. From this construction we find $F_{0}<0.52 @ 95 \% \mathrm{CL}$, and $F_{0}<0.94 @ 99 \% \mathrm{CL}$ in the dilepton only measurement.

The dilepton data are inconsistent with the standard model prediction $F_{0}=0.70$ at the 1 and $2 \sigma$ levels. We are unable to make a statement about $F_{0}$ at the $>3 \sigma$ level. However, the dilepton data are consistent with the lepton+jets data at the $2 \sigma$ level. Given this

level of agreement, it is reasonable to assume that we observe the same physical process in both samples. Because of this level of agreement, and because it was our original intent to measure $F_{0}$ in the combined samples, we will do so. 


\subsection{Result from the combined analysis}

Here we include in the likelihood function the three dilepton and the eight lepton+jets sub-samples. Using MIGRAD, we find the MLE for the combined measurement, $\hat{F}_{0}=$ 0.27. Figure 7.5 shows the projection of $-\log (\mathscr{L})$ along the $F_{0}$ axis, where the background fractions for each sub-sample are fixed to those values which minimize $-\log (\mathscr{L})$ when $F_{0}=0.27$. Figure 7.5 also shows the distribution of charged-lepton $p_{T}$ data for the three dilepton and eight lepton+jet samples overlaid with the total signal and background PDFs normalized according to their MLEs. The MLE for the combined measurement is consistent with the $2 \sigma$ intervals from the dilepton and lepton+jets only measurements.

Using the resolution function derived in section 6.1.2 for the combined measurement, and the estimate of systematic uncertainty from table 6.5 we construct Feldman Cousins confidence belts at the $68.3 \%, 95.4 \%$ and $99.7 \%$ confidence levels. These belts are plotted in figure 7.6, along with MLE for the combined measurement. From this construction we find $F_{0}=0.27_{-0.21}^{+0.35}$ (stat. + syst.) and $F_{0}<0.88 @ 95 \%$ CL in the combined analysis. This result is inconsistent with the standard model prediction $F_{0}=0.70$ at the $1 \sigma$ level; it is consistent with the standard model prediction at the $2 \sigma$ level. 


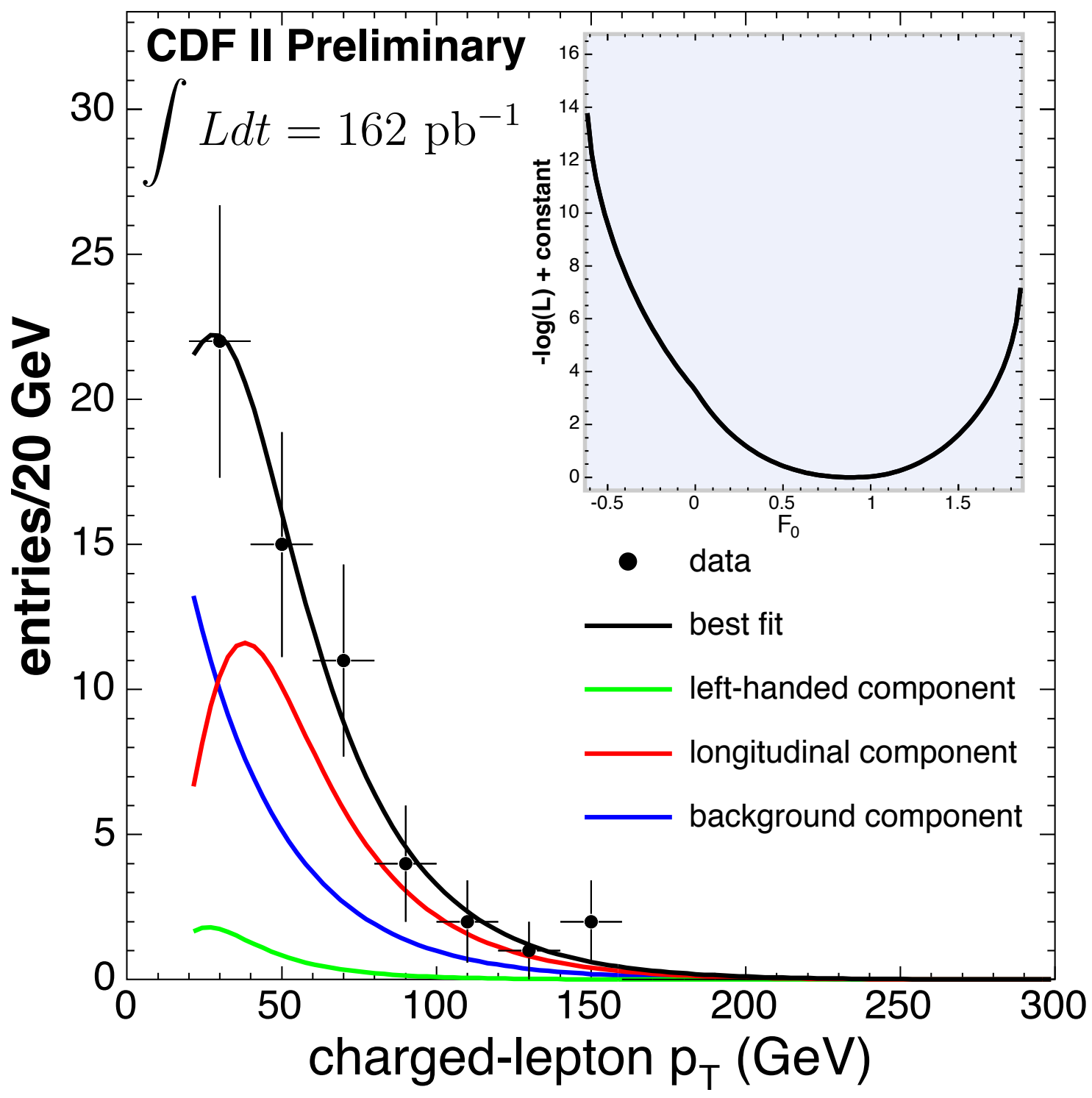

Figure 7.1: Result of the lepton+jets analysis. Left: Projection of $-\log (\mathscr{L})$ along the $F_{0}$ axis for the fit to the lepton + jets samples only. The background fractions are fixed to the values which absolutely maximize the likelihood function. The green band shows the MINOS $1 \sigma$ interval. Right: The distribution of charged-lepton $p_{T}$ for the lepton + lets samples overlaid with the total signal and background PDFs normalized according to their MLEs. 


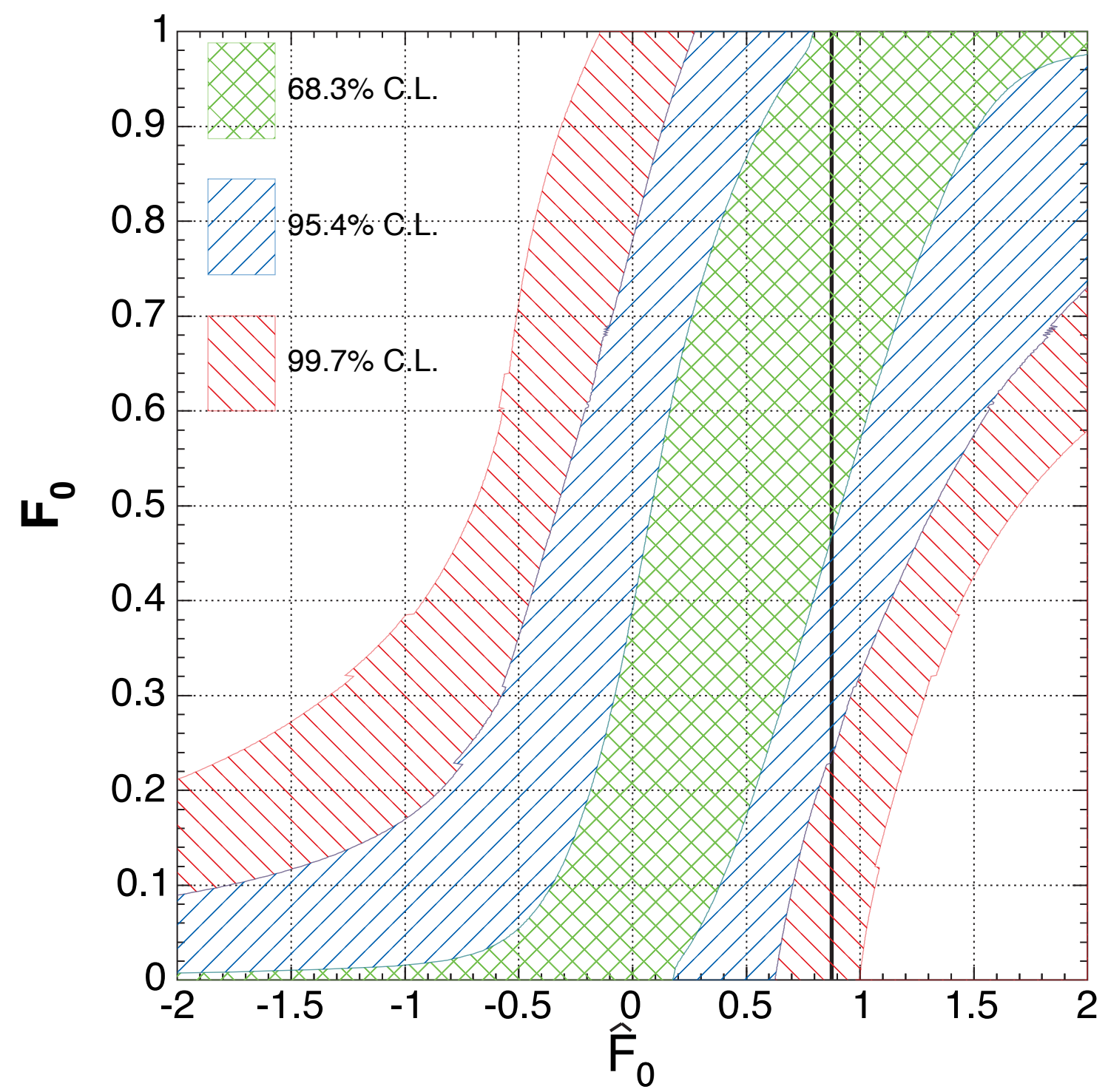

Figure 7.2: 1, 2 and $3 \sigma$ Feldman Cousins confidence belts for an experiment including the lepton+jets samples. These belts include systematic uncertainties. The thick vertical line indicates the experimental outcome, $\hat{F}_{0}=0.88$. 


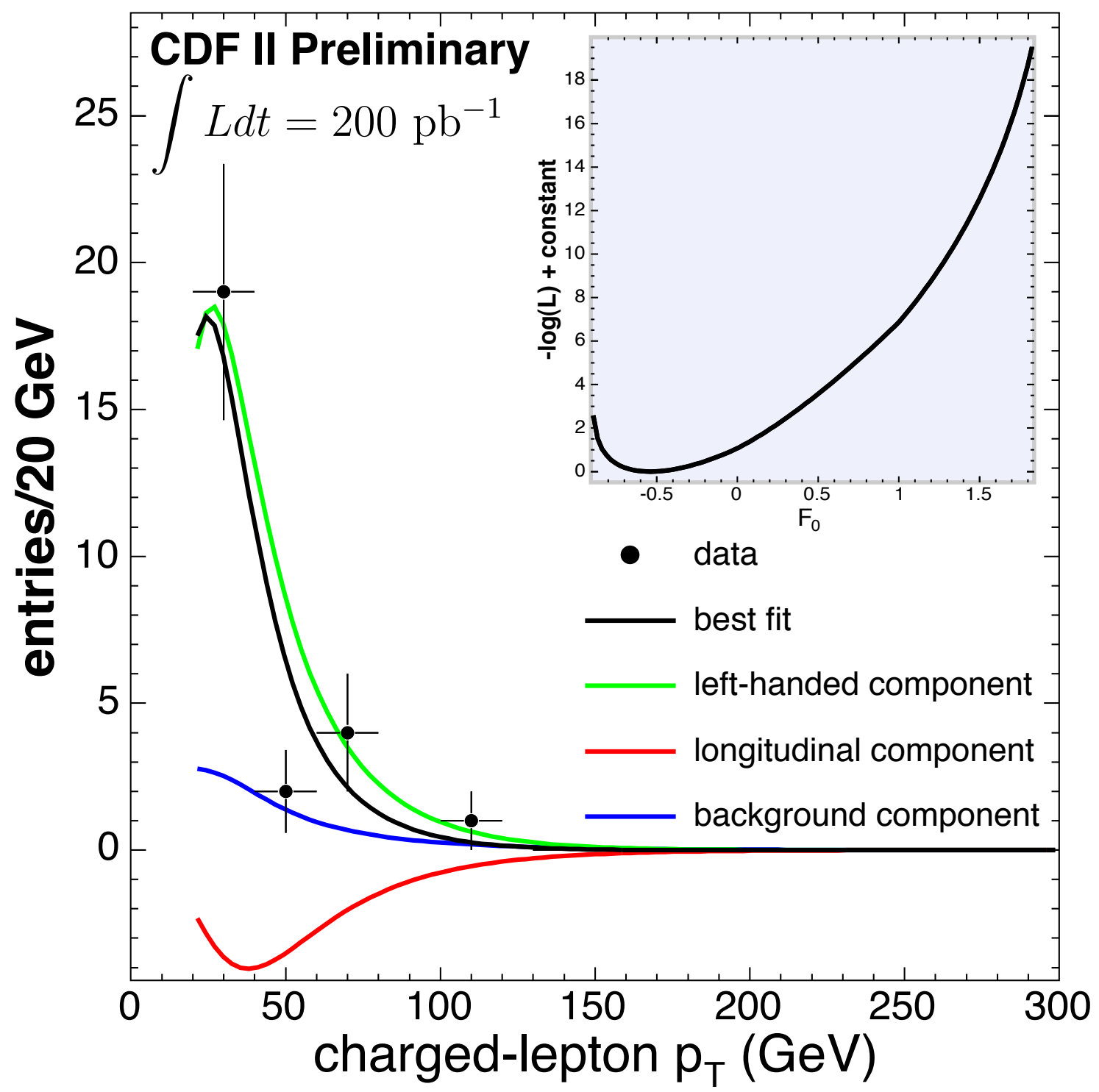

Figure 7.3: Result of the dilepton analysis. Left: Projection of $-\log (\mathscr{L})$ along the $F_{0}$ axis for the fit to the dilepton samples only. The background fractions are fixed to the values which absolutely maximize the likelihood function. The green band shows the MINOS $1 \sigma$ interval. Right: The distribution of chargedlepton $p_{T}$ for the dilepton samples overlaid with the total signal and background PDFs normalized according to their MLEs. 


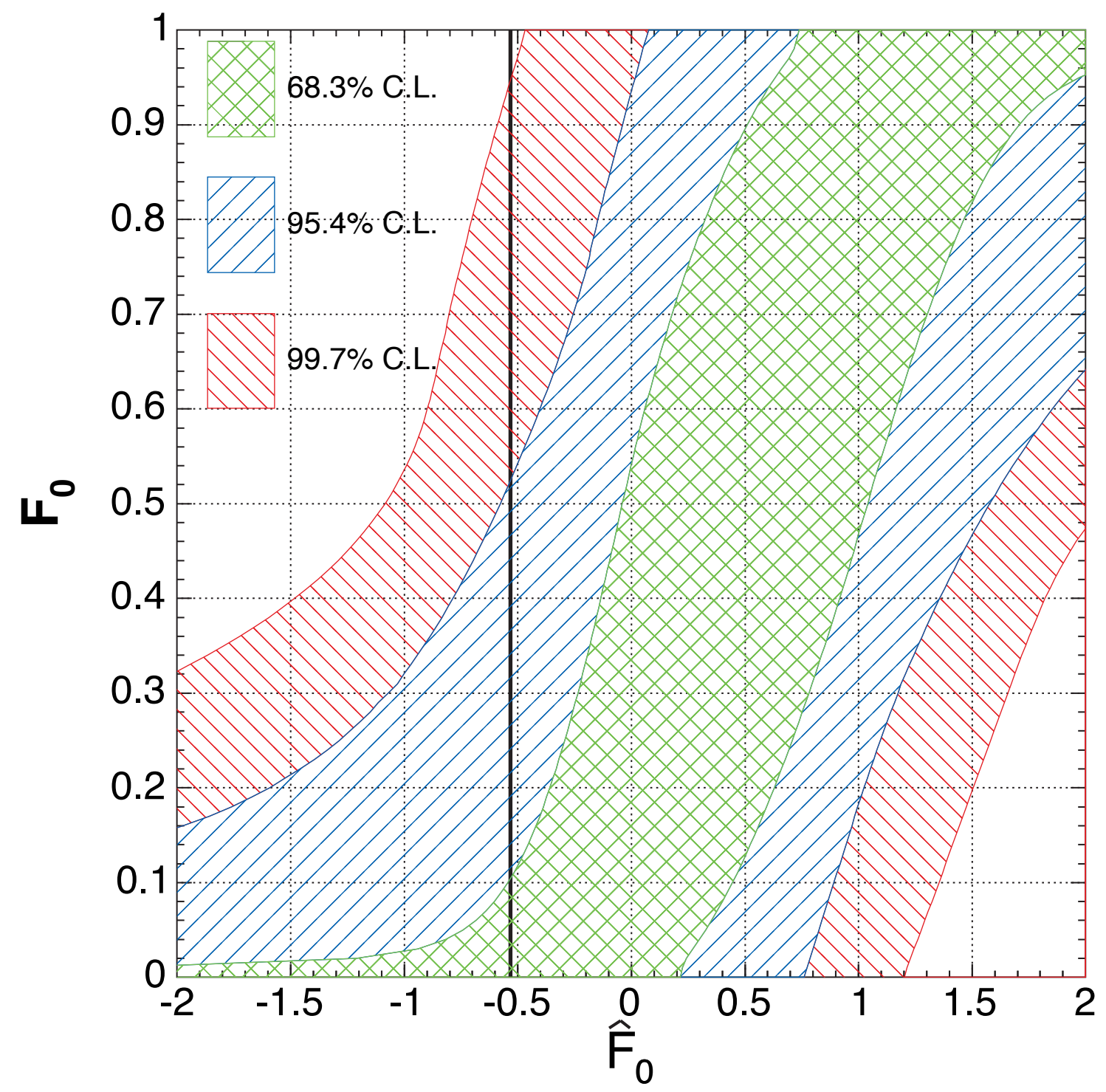

Figure 7.4: 1, 2 and $3 \sigma$ Feldman Cousins confidence belts for an experiment including the dilepton samples. These belts include systematic uncertainties. The thick vertical line indicates the experimental outcome, $\hat{F}_{0}=-0.54$. 


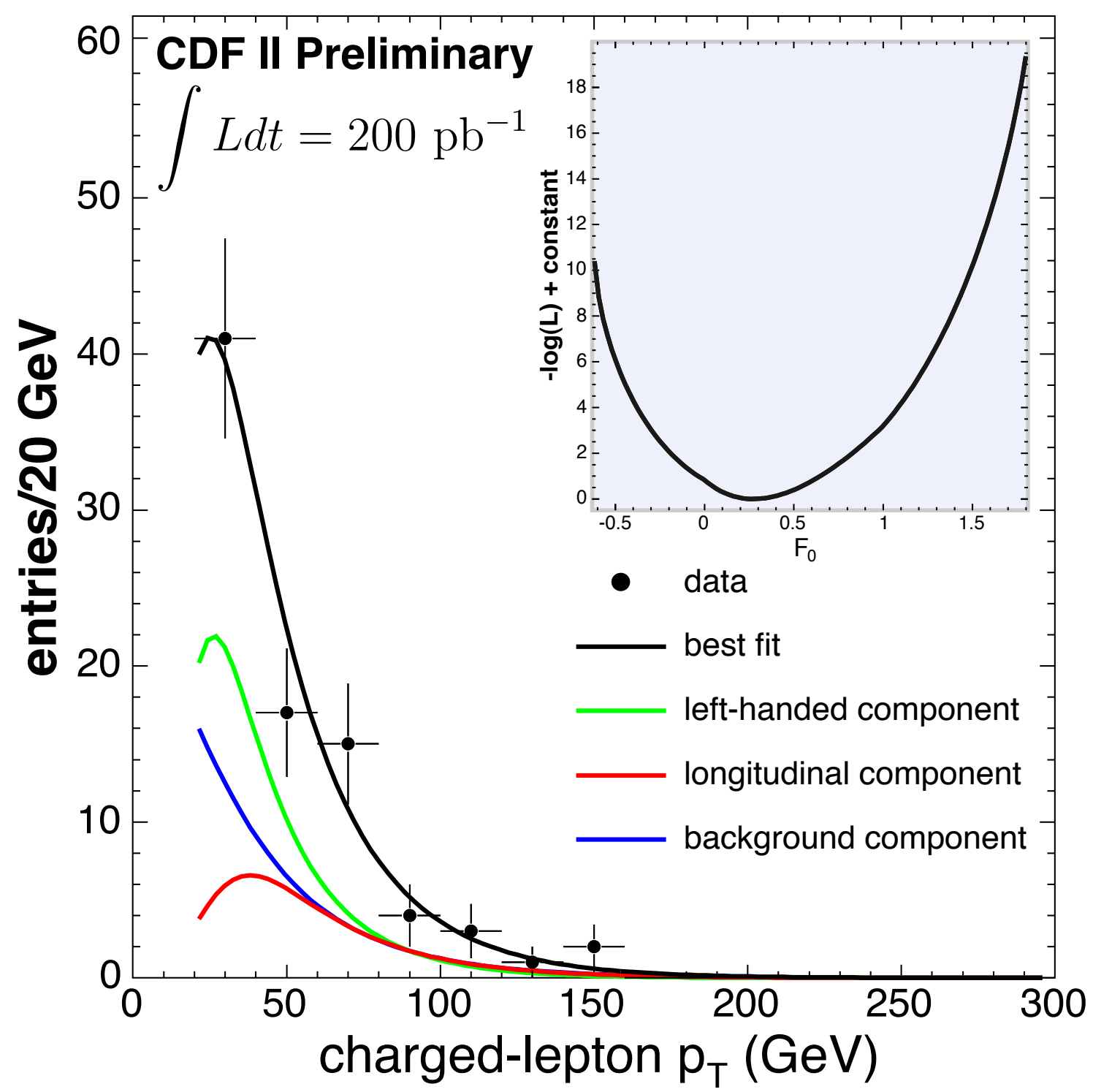

Figure 7.5: Result of the combined analysis. Left: Projection of $-\log (\mathscr{L})$ along the $F_{0}$ axis for the fit to the lepton+jets and dilepton samples. The background fractions are fixed to the values which absolutely maximize the likelihood function. The green band shows the MINOS $1 \sigma$ interval. Right: The distribution of charged-lepton $p_{T}$ for the lepton + jets and dilepton samples overlaid with the total signal and background PDFs normalized according to their MLEs. 


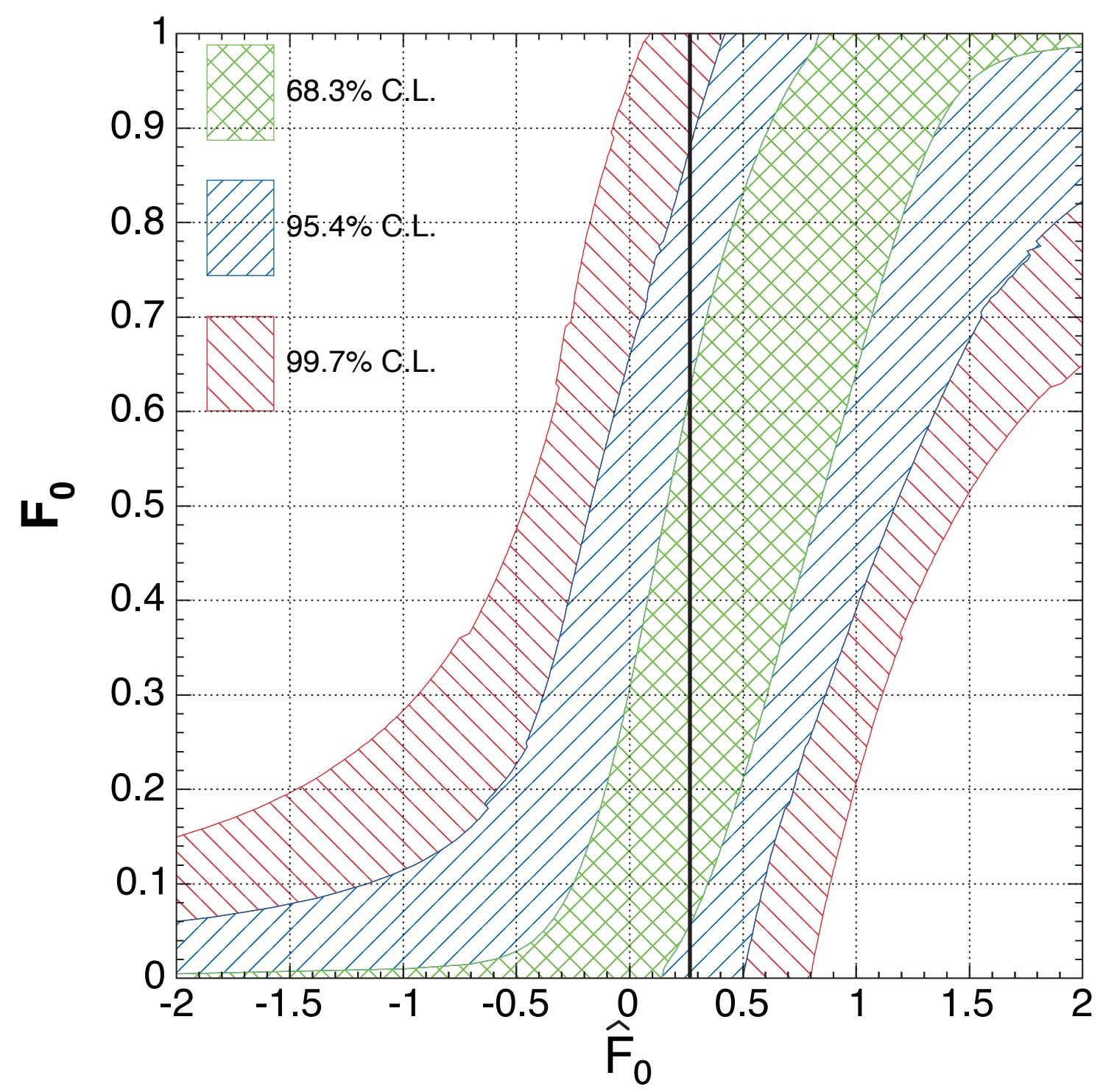

Figure 7.6: 1,2 and $3 \sigma$ Feldman Cousins confidence belts for an experiment including the lepton+jets and dilepton samples. These belts include systematic uncertainties. The thick vertical line indicates the experimental outcome, $\hat{F}_{0}=0.27$. 


\section{CHAPTER 8}

\section{Conclusions}

We have measured the fraction of longitudinally polarized $W$ bosons produced topquark decays.

The result of the analysis in single-lepton $t \bar{t}$ events is fully consistent with the Standard Model expectation.

The $2 \sigma$ discrepancy in the dilepton analysis is suggestive of new phenomena. However, given the significance of the discrepancy, any claim of new physics based on this analysis is

highly speculative. It is worth noting that another analysis of the kinematic properties of these data finds a similar discrepancy [63]. It will be interesting to see if this discrepancy persists as more data are collected during run II of the Tevatron.

In order to make a stronger statement about the nature of the $t W b$ coupling with this method, larger statistics are required. However alternative methods, particularly the matrix-element method developed at D $\varnothing[36,35]$, should be especially powerful, even with limited statistics. 
APPENDICES 


\section{APPENDIX A}

\section{Notation}

In this appendix we make explicit some of the notation used in Chapters 1 and 2. We follow conventions used in the textbook by Peskin and Schroeder [1], and the paper by Kane, Ladinsky and Yuan [23].

We use the metric

$$
g^{\mu \nu}=\left(\begin{array}{cccc}
1 & 0 & 0 & 0 \\
0 & -1 & 0 & 0 \\
0 & 0 & -1 & 0 \\
0 & 0 & 0 & -1
\end{array}\right)
$$

In a spherical coordinates, the general form of an object's four-momentum is

$$
p^{\mu}=(E,|\vec{p}| \sin \theta \cos \phi,|\vec{p}| \sin \theta \sin \phi,|\vec{p}| \cos \theta)
$$

with $E^{2}-|\vec{p}|^{2}=m^{2}$. The left-handed, $(\lambda=-1)$ longitudinal $(\lambda=0)$ and the right-handed $(\lambda=+1)$ polarization states for a spin-1 field are

$$
\begin{aligned}
\epsilon_{(-)}^{\mu} & =\frac{e^{i \phi}}{\sqrt{2}}(0,-\cos \phi \cos \theta-i \sin \phi,-\sin \phi \cos \theta+i \cos \phi,-\sin \theta) \\
\epsilon_{(0)}^{\mu} & =\frac{1}{m}(|\vec{p}|, E \sin \theta \cos \phi, E \sin \theta \sin \phi, E \cos \theta) \\
\epsilon_{(+)}^{\mu} & =\frac{e^{i \phi}}{\sqrt{2}}(0, \cos \phi \cos \theta-i \sin \phi, \sin \phi \cos \theta+i \cos \phi, \sin \theta) .
\end{aligned}
$$

The longitudinal polarization state is forbidden for a massless spin-1 field. 
In the Weyl basis, Dirac spinors have the form

$$
\psi=\left(\begin{array}{l}
\psi_{+} \\
\psi_{-}
\end{array}\right)
$$

For fermions,

$$
\psi_{ \pm}=\left\{\begin{array}{l}
u_{ \pm}^{(\lambda=1)}=\sqrt{E \pm|\vec{p}|} \xi_{\frac{1}{2}} \\
u_{ \pm}^{(\lambda=-1)}=\sqrt{E \mp|\vec{p}|} \xi_{-\frac{1}{2}}
\end{array}\right.
$$

For anti-fermions,

$$
\psi_{ \pm}=\left\{\begin{array}{l}
v_{ \pm}^{(\lambda=1)}= \pm \sqrt{E \mp|\vec{p}|} \xi_{-\frac{1}{2}} \\
v_{ \pm}^{(\lambda=-1)}=\mp \sqrt{E \pm|\vec{p}|} \xi_{\frac{1}{2}}
\end{array}\right.
$$

The two-component spinors $\xi_{\lambda / 2}$ are eigenvectors of the helicity operator

$$
h=\hat{p} \cdot \overrightarrow{\mathbf{S}}
$$

eigenvalue $\lambda=+1$ corresponds to the "spin-up" eigenstate, $\lambda=-1$ corresponds to the "spin-down" eigenstate.

$$
\xi_{\frac{1}{2}}=\left(\begin{array}{c}
\cos \theta / 2 \\
e^{i \phi} \sin \theta / 2
\end{array}\right), \quad \xi_{-\frac{1}{2}}=\left(\begin{array}{c}
-e^{-i \phi} \sin \theta / 2 \\
\cos \theta / 2
\end{array}\right) .
$$

In the Weyl basis, the gamma matrices have the form

$$
\gamma^{0}=\left(\begin{array}{ll}
0 & 1 \\
1 & 0
\end{array}\right), \quad \gamma^{j}=\left(\begin{array}{cc}
0 & -\sigma_{j} \\
\sigma_{j} & 0
\end{array}\right), \quad \gamma^{5}=\gamma_{5}=\left(\begin{array}{cc}
1 & 0 \\
0 & -1
\end{array}\right),
$$

where $\sigma_{j}$ are the $2 \times 2$ Pauli matrices

$$
\sigma_{1}=\left(\begin{array}{cc}
0 & 1 \\
1 & 0
\end{array}\right), \quad \sigma_{2}=\left(\begin{array}{cc}
0 & -i \\
i & 0
\end{array}\right), \quad \sigma_{3}=\left(\begin{array}{cc}
1 & 0 \\
0 & -1
\end{array}\right)
$$


The chirality projection operators are defined by

$$
P_{ \pm}=\frac{1}{2}\left(1 \pm \gamma^{5}\right)
$$

The operator $P_{+}\left(P_{-}\right)$projects out the right-handed (left-handed) component of the Weyl spinor.

$$
\begin{aligned}
& P_{-} \psi=\left(\begin{array}{ll}
0 & 0 \\
0 & 1
\end{array}\right)\left(\begin{array}{l}
\psi_{+} \\
\psi_{-}
\end{array}\right)=\left(\begin{array}{l}
0 \\
\psi_{-}
\end{array}\right) \\
& \bar{\psi} P_{+}=\left(\psi_{+}^{\dagger} \psi_{-}^{\dagger}\right)\left(\begin{array}{ll}
0 & 1 \\
1 & 0
\end{array}\right)\left(\begin{array}{ll}
1 & 0 \\
0 & 0
\end{array}\right)=\left(\psi_{-}^{\dagger} 0\right) .
\end{aligned}
$$




\section{APPENDIX B}

\section{The CDF II Collaboration}

D. Acosta, J. Adelman, T. Affolder, T. Akimoto, M.G. Albrow, D. Ambrose, S. Amerio, D. Amidei, A. Anastassov, K. Anikeev, A. Annovi, J. Antos, M. Aoki, G. Apollinari, T. Arisawa, J-F. Arguin, A. Artikov, W. Ashmanskas, A. Attal, F. Azfar, P. AzziBacchetta, N. Bacchetta, H. Bachacou, W. Badgett, A. Barbaro-Galtieri, G.J. Barker, V.E. Barnes, B.A. Barnett, S. Baroiant, M. Barone, G. Bauer, F. Bedeschi, S. Behari, S. Belforte, G. Bellettini, J. Bellinger, E. Ben-Haim, D. Benjamin, A. Beretvas, A. Bhatti, M. Binkley, D. Bisello, M. Bishai, R.E. Blair, C. Blocker, K. Bloom, B. Blumenfeld, A. Bocci, A. Bodek, G. Bolla, A. Bolshov, P.S.L. Booth, D. Bortoletto, J. Boudreau, S. Bourov, C. Bromberg, E. Brubaker, J. Budagov, H.S. Budd, K. Burkett, G. Busetto, P. Bussey, K.L. Byrum, S. Cabrera, M. Campanelli, M. Campbell, A. Canepa, M. Casarsa, D. Carlsmith, S. Carron, R. Carosi, M. Cavalli-Sforza, A. Castro, P. Catastini, D. Cauz, A. Cerri, C. Cerri, L. Cerrito, J. Chapman, C. Chen, Y.C. Chen, M. Chertok, G. Chiarelli,

G. Chlachidze, F. Chlebana, I. Cho, K. Cho, D. Chokheli, M.L. Chu, S. Chuang, J.Y. Chung, W-H. Chung, Y.S. Chung, C.I. Ciobanu, M.A. Ciocci, A.G. Clark, D. Clark, M. Coca, A. Connolly, M. Convery, J. Conway, B. Cooper, M. Cordelli, G. Cortiana, J. Cranshaw, J. Cuevas, R. Culbertson, C. Currat, D. Cyr, D. Dagenhart, S. Da Ronco, S. D'Auria, P. de Barbaro, S. De Cecco, G. De Lentdecker, S. Dell'Agnello, M. Dell'Orso, S. De- 
mers, L. Demortier, M. Deninno, D. De Pedis, P.F. Derwent, C. Dionisi, J.R. Dittmann, P. Doksus, A. Dominguez, S. Donati, M. Donega, J. Donini, M. D’Onofrio, T. Dorigo, V. Drollinger, K. Ebina, N. Eddy, R. Ely, R. Erbacher, M. Erdmann, D. Errede, S. Errede, R. Eusebi, H-C. Fang, S. Farrington, I. Fedorko, R.G. Feild, M. Feindt, J.P. Fernandez, C. Ferretti, R.D. Field, I. Fiori, G. Flanagan, B. Flaugher, L.R. Flores-Castillo, A. Foland, S. Forrester, G.W. Foster, M. Franklin, J.C. Freeman, H. Frisch, Y. Fujii, I. Furic, A. Gajjar, A. Gallas, J. Galyardt, M. Gallinaro, M. Garcia-Sciveres, A.F. Garfinkel, C. Gay, H. Gerberich, D.W. Gerdes, E. Gerchtein, S. Giagu, P. Giannetti, A. Gibson, K. Gibson, C. Ginsburg, K. Giolo, M. Giordani, G. Giurgiu, V. Glagolev, D. Glenzinski, M. Gold, N. Goldschmidt, D. Goldstein, J. Goldstein, G. Gomez, G. Gomez-Ceballos, M. Goncharov, O. González, I. Gorelov, A.T. Goshaw, Y. Gotra, K. Goulianos, A. Gresele, M. Griffiths, C. Grosso-Pilcher, U. Grundler, M. Guenther, J. Guimaraes da Costa, C. Haber, K. Hahn, S.R. Hahn, E. Halkiadakis, A. Hamilton, B-Y. Han, R. Handler, F. Happacher, K. Hara, M. Hare, R.F. Harr, R.M. Harris, F. Hartmann, K. Hatakeyama, J. Hauser, C. Hays, H. Hayward, E. Heider, B. Heinemann, J. Heinrich, M. Hennecke, M. Herndon, C. Hill, D. Hirschbuehl, A. Hocker, K.D. Hoffman, A. Holloway, S. Hou, M.A. Houlden, B.T. Huffman, Y. Huang, R.E. Hughes, J. Huston, K. Ikado, J. Incandela, G. Introzzi, M. Iori, Y. Ishizawa, C. Issever, A. Ivanov, Y. Iwata, B. Iyutin, E. James, D. Jang, J. Jarrell, D. Jeans, H. Jensen, E.J. Jeon, M. Jones, K.K. Joo, S. Jun, T. Junk, T. Kamon, J. Kang, M. Karagoz Unel, P.E. Karchin, S. Kartal, Y. Kato, Y. Kemp, R. Kephart, U. Kerzel, V. Khotilovich, B. Kilminster, D.H. Kim, H.S. Kim, J.E. Kim, M.J. Kim, M.S. Kim, S.B. Kim, S.H. Kim, T.H. Kim, Y.K. Kim, B.T. King, M. Kirby, L. Kirsch, S. Klimenko, B. Knuteson, B.R. Ko, H. Kobayashi, P. Koehn, D.J. Kong, K. Kondo, J. Konigsberg, K. Kordas, A. Korn, A. Korytov, K. Kotelnikov, A.V. Kotwal, A. Kovalev, J. Kraus, I. Kravchenko, A. Kreymer, J. Kroll, M. Kruse, V. Krutelyov, 
S.E. Kuhlmann, N. Kuznetsova, A.T. Laasanen, S. Lai, S. Lami, S. Lammel, J. Lancaster, M. Lancaster, R. Lander, K. Lannon, A. Lath, G. Latino, R. Lauhakangas, I. Lazzizzera, Y. Le, C. Lecci, T. LeCompte, J. Lee, J. Lee, S.W. Lee, R. Lefèvre, N. Leonardo, S. Leone, J.D. Lewis, K. Li, C. Lin, C.S. Lin, M. Lindgren, T.M. Liss, D.O. Litvintsev, T. Liu, Y. Liu, N.S. Lockyer, A. Loginov, M. Loreti, P. Loverre, R-S. Lu, D. Lucchesi, P. Lujan, P. Lukens, G. Lungu, L. Lyons, J. Lys, R. Lysak, D. MacQueen, R. Madrak, K. Maeshima, P. Maksimovic, L. Malferrari, G. Manca, R. Marginean, M. Martin, A. Martin, V. Martin, M. Martínez, T. Maruyama, H. Matsunaga, M. Mattson, P. Mazzanti, K.S. McFarland, D. McGivern, P.M. McIntyre, P. McNamara, R. NcNulty, S. Menzemer, A. Menzione, P. Merkel, C. Mesropian, A. Messina, T. Miao, N. Miladinovic, L. Miller, R. Miller, J.S. Miller, R. Miquel, S. Miscetti, G. Mitselmakher, A. Miyamoto, Y. Miyazaki, N. Moggi, B. Mohr, R. Moore, M. Morello, A. Mukherjee, M. Mulhearn, T. Muller, R. Mumford, A. Munar, P. Murat, J. Nachtman, S. Nahn, I. Nakamura, I. Nakano, A. Napier, R. Napora, D. Naumov, V. Necula, F. Niell, J. Nielsen, C. Nelson, T. Nelson, C. Neu, M.S. Neubauer, C. Newman-Holmes, A-S. Nicollerat, T. Nigmanov, L. Nodulman, O. Norniella, K. Oesterberg, T. Ogawa, S.H. Oh, Y.D. Oh, T. Ohsugi, T. Okusawa, R. Oldeman, R. Orava, W. Orejudos, C. Pagliarone, F. Palmonari, R. Paoletti, V. Papadimitriou, S. Pashapour, J. Patrick, G. Pauletta, M. Paulini, T. Pauly, C. Paus, D. Pellett, A. Penzo, T.J. Phillips, G. Piacentino, J. Piedra, K.T. Pitts, C. Plager, A. Pompoš, L. Pondrom, G. Pope, O. Poukhov, F. Prakoshyn, T. Pratt, A. Pronko, J. Proudfoot, F. Ptohos, G. Punzi, J. Rademacker, A. Rakitine, S. Rappoccio, F. Ratnikov, H. Ray, A. Reichold, B. Reisert, V. Rekovic, P. Renton, M. Rescigno, F. Rimondi, K. Rinnert, L. Ristori, W.J. Robertson, A. Robson, T. Rodrigo, S. Rolli, L. Rosenson, R. Roser, R. Rossin, C. Rott, J. Russ, A. Ruiz, D. Ryan, H. Saarikko, S. Sabik, A. Safonov, R. St. Denis, W.K. Sakumoto, G. Salamanna, D. Saltzberg, C. Sanchez, 
A. Sansoni, L. Santi, S. Sarkar, K. Sato, P. Savard, A. Savoy-Navarro, P. Schlabach, E.E. Schmidt, M.P. Schmidt, M. Schmitt, L. Scodellaro, A. Scribano, F. Scuri, A. Sedov, S. Seidel, Y. Seiya, F. Semeria, L. Sexton-Kennedy, I. Sfiligoi, M.D. Shapiro, T. Shears, P.F. Shepard, M. Shimojima, M. Shochet, Y. Shon, I. Shreyber, A. Sidoti, J. Siegrist, M. Siket, A. Sill, P. Sinervo, A. Sisakyan, A. Skiba, A.J. Slaughter, K. Sliwa, D. Smirnov, J.R. Smith, F.D. Snider, R. Snihur, S.V. Somalwar, J. Spalding, M. Spezziga, L. Spiegel, F. Spinella, M. Spiropulu, P. Squillacioti, H. Stadie, A. Stefanini, B. Stelzer, O. StelzerChilton, J. Strologas, D. Stuart, A. Sukhanov, K. Sumorok, H. Sun, T. Suzuki, A. Taffard, R. Tafirout, S.F. Takach, H. Takano, R. Takashima, Y. Takeuchi, K. Takikawa, M. Tanaka, R. Tanaka, N. Tanimoto, S. Tapprogge, M. Tecchio, P.K. Teng, K. Terashi, R.J. Tesarek, S. Tether, J. Thom, A.S. Thompson, E. Thomson, P. Tipton, V. Tiwari, S. Tkaczyk, D. Toback, K. Tollefson, T. Tomura, D. Tonelli, M. Tönnesmann, S. Torre, D. Torretta, S. Tourneur, W. Trischuk, J. Tseng, R. Tsuchiya, S. Tsuno, D. Tsybychev, N. Turini, M. Turner, F. Ukegawa, T. Unverhau, S. Uozumi, D. Usynin, L. Vacavant, A. Vaiciulis, A. Varganov, E. Vataga, S. Vejcik III, G. Velev, V. Veszpremi, G. Veramendi, T. Vickey, R. Vidal, I. Vila, R. Vilar, I. Vollrath, I. Volobouev, M. von der Mey, P. Wagner, R.G. Wagner, R.L. Wagner, W. Wagner, R. Wallny, T. Walter, T. Yamashita, K. Yamamoto, Z. Wan, M.J. Wang, S.M. Wang, A. Warburton, B. Ward, S. Waschke, D. Waters, T. Watts, M. Weber, W.C. Wester III, B. Whitehouse, A.B. Wicklund, E. Wicklund, H.H. Williams, P. Wilson, B.L. Winer, P. Wittich, S. Wolbers, M. Wolter, M. Worcester, S. Worm, T. Wright, X. Wu, F. Würthwein, A. Wyatt, A. Yagil, U.K. Yang, W. Yao, G.P. Yeh, K. Yi, J. Yoh, P. Yoon, K. Yorita, T. Yoshida, I. Yu, S. Yu, Z. Yu, J.C. Yun, L. Zanello, A. Zanetti, I. Zaw, F. Zetti, J. Zhou, A. Zsenei, S. Zucchelli (634 authors listed) 


\section{BIBLIOGRAPHY}


[1] M. E. Peskin and D. V. Schroeder, An Introduction to Quantum Field Theory, Perseus Books, Boston, 1995. 1, 100

[2] G. Kane, Modern Elementary Particle Physics, Perseus Books, Boston, 1993. 1

[3] W. Greiner and A. Schäefer, Quantum Chromodynamics, Springer-Verlag, Berlin, 1 ed., 1995. 1

[4] W. Greiner and B. Müller, Gauge Theory of Weak Interactions, Springer-Verlag, Berlin, 2 ed., 1996. 1

[5] V. D. Barger and R. J. N. Phillips, Collider Physics, Perseus Publishing, Boston, 1996. 1

[6] H. D. Politzer, Asymptotic freedom: An approach to strong interactions, Phys. Rept., 14 (1974), pp. $129-180.2$

[7] D. J. Gross And F. WilczeK, Asymptotically free gauge theories, Phys. Rev., D8 (1973), pp. 36333652. 2

[8] S. Weinberg, A model of leptons, Phys. Rev. Lett., 19 (1967), pp. 1264-1266. 2

[9] A. Salam And J. C. Ward, Electromagnetic and weak interactions, Phys. Lett., 13 (1964). 2

[10] S. L. Glashow, Partial symmetries of weak interactions, Nucl. Phys., 22 (1961). 2

[11] A. Salam, Weak and electromagnetic interactions, in Elementary Particle Theory, N. Svartholm, ed., Stockholm, 1968, Amkvist and Wiksell, p. 367. 2

[12] P. W. Higgs, Broken symmetries, massless particles and gauge fields, Phys. Lett., 12 (1964), pp. 132-133. 2, 4

[13] — Broken symmetries and the masses of gauge bosons, Phys. Rev. Lett., 13 (1964), pp. 508-509. 2,4

[14] G. 'т Hooft, Renormalization of massless Yang-Mills fields, Nucl. Phys., B33 (1971), pp. 173-199. 4

[15] _ Renormalizable lagrangians for massive Yang-Mills fields, Nucl. Phys., B35 (1971), pp. 167188. 4

[16] G. 'т Hooft and M. J. G. Veltman, Regularization and renormalization of gauge fields, Nucl. Phys., B44 (1972), pp. 189-213. 4

[17] S. Eidelman et Al., Review of particle physics, Phys. Lett., B592 (2004). 8, 9, 13, 52

[18] P. AzZi ET AL., Combination of CDF and DØ results on the top-quark mass, tech. rep., The CDF Collaboration, the DØ Collaboration, and the Tevatron Electroweak Working Group, 2004. 8

[19] Y. FukUda ET AL., Evidence for oscillation of atmospheric neutrinos, Phys. Rev. Lett., 81 (1998), p. 15.9

[20] S. FukUdA ET AL., Constraints on neutrino oscillations using 1258 days of super-kamiokande solar neutrino data, Phys. Rev. Lett., 86 (2001), pp. 5656-5660. 9

[21] M. Kobayashi And T. Maskawa, CP violation in the renormalizable theory of weak interaction, Prog. Theor. Phys., 49 (1973), pp. 652-657. 13

[22] R. D. Peccei And X. Zhang, Dynamical symmetry breaking and universality breakdown, Nucl. Phys., B337 (1990), pp. 269-283. 16 
[23] G. L. Kane, G. A. Ladinsky, And C. P. Yuan, Using the top quark for testing standard model polarization and CP predictions, Phys. Rev., D45 (1992), pp. 124-141. 16, 17, 100

[24] F. Larios, M. A. Perez, And C. P. Yuan, Analysis of tbW and ttZ couplings from CLEO and LEP/SLC data, Phys. Lett., B457 (1999), pp. 334-340. 17

[25] M. Frigeni And R. Rattazzi, Testing an anomalous top quark via precision electroweak measurements, Phys. Lett., B269 (1991), pp. 412-418. 17

[26] J. Pumplin, D. R. Stump, J. Huston, H. L. Lai, P. Nadolsky, and W. K. Tung, New generation of parton distributions with uncertainties from global QCD analysis, JHEP, 07 (2002), p. $12.20,21$

[27] Y. L. Dokshitzer, Calculation of the structure functions for deep inelastic scattering and $e^{+} e^{-}$ annihilation by perturbation theory in quantum chromodynamics. (in Russian), Sov. Phys. JETP, 46 (1977), pp. 641-653. 21

[28] V. N. Gribov and L. N. Lipatov, $e^{+} e^{-}$pair annihilation and deep inelastic ep scattering in perturbation theory, Sov. J. Nucl. Phys., 15 (1972), pp. 675-684. 21

[29] G. Altarelli and G. Parisi, Asymptotic freedom in parton language, Nucl. Phys., B126 (1977), p. 298. 21

[30] M. Cacciari, S. Frixione, M. L. Mangano, P. Nason, and G. Ridolfi, The t $t \bar{t}$ cross-section at $1.8 \mathrm{TeV}$ and $1.96 \mathrm{TeV}$ : A study of the systematics due to parton densities and scale dependence, JHEP, 04 (2004), p. 68. 21, 56

[31] T. N. Vickey, Measurement of $W$ boson polarization in top quark decay, $\mathrm{PhD}$ thesis, University of Illinois at Urbana-Champaign, 2004. 27

[32] B. Kilminster, Testing $V$-A in top decay at $C D F$ at $\sqrt{s}=1.8 \mathrm{TeV}$, AIP Conf. Proc., 698 (2004), pp. $218-221.28$

[33] — A search for non-standard model $W$ helicity in top quark decays, $\mathrm{PhD}$ thesis, University of Rochester, 2003. 28

[34] D. Acosta And others., Measurement of the $W$ boson polarization in top decay, Phys. Rev., D71 (2005), p. 031101. 28

[35] V. M. Abazov et Al., Helicity of the $W$ boson in lepton+jets $t \bar{t}$ events, preprint, (2004). 28, 98

[36] M. F. Canelli, Helicity of the $W$ boson in single-lepton t $\bar{t}$ events, $\mathrm{PhD}$ thesis, University of Rochester, 2003. 28, 98

[37] T. Affolder et Al., Measurement of the helicity of $W$ bosons in top quark decays, Phys. Rev. Lett., 84 (2000), pp. 216-221. 28

[38] D. J. Winn, Measurement of the helicity of $W$ bosons in top quark decays, PhD thesis, University of Michigan, 2000. 28

[39] G. Corcella et AL., HERWIG 6: An event generator for hadron emission reactions with interfering gluons (including supersymmetric processes), JHEP, 01 (2001), p. 10. 30, 61

[40] E. Guillian, Top quark decay kinematics in fully reconstructed t $\bar{t}$ events in the e or $\mu+\mathbb{E}_{T}+\geq 4$ jet decay channel, $\mathrm{PhD}$ thesis, University of Michigan, 1999. 30, 61

[41] G. Corcella. personal communication, 2002. 30

[42] D. McGinnis ET AL., The run II handbook, Tech. Rep. http://www-ad.fnal.gov/runII/, Fermilab Accelerator Division, 2000. 31 
[43] R. Blair ET AL., The CDF-II detector: technical design report, Tech. Rep. FERMILAB-PUB-96390-E, Fermi National Accelerator Laboratory, 1996. 31

[44] D. Acosta ET AL., Measurement of the $J / \psi$ meson and b-hadron production cross sections in $p \bar{p}$ collisions at $\sqrt{s}=1960 \mathrm{GeV}$, Phys. Rev. D, 71 (2005), p. 25. 31

[45] T. Affolder et Al., CDF central outer tracker, Nucl. Instrum. Meth., A526 (2004), pp. 249-299. 37,40

[46] A. Sill, CDF run II silicon tracking projects, Nucl. Instrum. Meth., A447 (2000), pp. 1-8. 37, 39

[47] C. S. HILL, Operational experience and performance of the CDF II silicon detector, Nucl. Instrum. Meth., A530 (2004), pp. 1-6. 38

[48] A. AfFolder ET AL., Intermediate silicon layers detector for the CDF experiment, Nucl. Instrum. Meth., A453 (2000), pp. 84-88. 40

[49] L. BALKa ET AL., The CDF central electromagnetic calorimeter, Nucl. Instrum. Meth., A267 (1988), p. 272.44

[50] S. Bertolucci et Al., The CDF central and endwall hadron calorimeter, Nucl. Instrum. Meth., A267 (1988), p. 301. 44

[51] A. Artikov et Al., Design and construction of new central and forward muon counters for CDF II, Nucl. Instrum. Meth., A538 (2005), pp. 358-371. 46

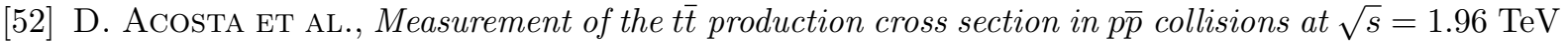
using lepton + jets events with secondary vertex b-tagging, Phys. Rev. D, 71 (2005), p. 052003. 50, 55

[53] L. D. Landau, On the energy loss of fast particles by ionisation, in Collected Papers of L.D. Landau, D. ter Haar, ed., Gordon and Breach, New York, 2 ed., 1967, pp. 417-424. 62

[54] K. S. KölBig AND B. SchorR, A program package for the Landau distribution, Comput. Phys. Commun., 31 (1984), pp. 97-111. 62

[55] http://cernlib.web.cern.ch/cernlib/libraries.html. 62

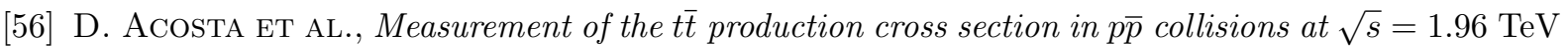
using dilepton events, Phys. Rev. Lett., 93 (2004), p. 142001. 62

[57] J. Nielsen, Y. K. Kim, L. Tompkins, and G. Veramendi, Trigger efficiencies for high $E_{T}$ electrons, Tech. Rep. 6234, The CDF II Collaboration, 2004. 71

[58] G. Chiarelli, I. Fedorko, And A. Sidoti, Trigger efficiencies for plug electrons in run II, Tech. Rep. 6864, The CDF II Collaboration, 2004. 71

[59] IEEE TASK P754, ANSI/IEEE 754-1985, Standard for Binary Floating-Point Arithmetic, IEEE, New York, 12 1985. Available from the IEEE Service Center, Piscataway, NJ, USA. 72

[60] F. James AND M. Roos, 'MINUIT' a system for function minimization and analysis of the parameter errors and correlations, Comput. Phys. Commun., 10 (1975), pp. 343-367. 72

[61] G. J. Feldman and R. D. Cousins, A unified approach to the classical statistical analysis of small signals, Phys. Rev., D57 (1998), pp. 3873-3889. 73

[62] K. BLoom, Estimating QCD backgrounds to W production, Tech. Rep. 6559, The CDF II Collaboration, 2003. 86

[63] A. G. Ivanov, Search for anomalous kinematics of top dilepton events $p \bar{p}$ collisions at $\sqrt{s}=$ $1.96 \mathrm{TeV}, \mathrm{PhD}$ thesis, University of Rochester, 2004. 98 